Florida International University FIU Digital Commons

\title{
Multidimensional Leadership: Masculine and Feminine Leadership Approaches in Public Education
}

Joseph P. Eberhard

Florida International University, JosephEberhard@hotmail.com

DOI: $10.25148 /$ etd.FIDC001946

Follow this and additional works at: https://digitalcommons.fiu.edu/etd

3 Part of the Educational Leadership Commons, Elementary and Middle and Secondary Education Administration Commons, Other Education Commons, Other Educational Administration and Supervision Commons, Other Social and Behavioral Sciences Commons, and the Social and Philosophical Foundations of Education Commons

\section{Recommended Citation}

Eberhard, Joseph P., "Multidimensional Leadership: Masculine and Feminine Leadership Approaches in Public Education" (2017). FIU Electronic Theses and Dissertations. 3392.

https://digitalcommons.fiu.edu/etd/3392 
FLORIDA INTERNATIONAL UNIVERSITY

Miami, Florida

MULTIDIMENSIONAL LEADERSHIP: MASCULINE AND FEMININE

LEADERSHIP APPROACHES IN PUBLIC EDUCATION

A dissertation submitted in partial fulfillment of the

requirements for the degree of

DOCTOR OF EDUCATION

in

EDUCATIONAL ADMINISTRATION AND SUPERVISION

by

Joseph P. Eberhard

2017 
To: Dean Michael R. Heithaus,

College of Arts, Sciences and Education

This dissertation, written by Joseph P. Eberhard, and entitled Multidimensional Leadership: Masculine and Feminine Leadership Approaches in Public Education, having been approved in respect to style and intellectual content, is referred to you for judgment.

We have read this dissertation and recommend that it be approved.

$\begin{array}{r}\hline \text { Mido Chang } \\ \hline \text { Laura H. Dinehart } \\ \hline \text { Sarah A. Mathews } \\ \hline \text { Peter J. Cistone, Major Professor }\end{array}$

Date of Defense: May 16, 2017

The dissertation of Joseph P. Eberhard is approved.

Dean Michael R. Heithaus

College of Arts, Sciences and Education

Andrés G. Gil

Vice President for Research and Economic Development

And Dean of the University Graduate School

Florida International University, 2017 
(C) Copyright 2017 by Joseph P. Eberhard

All rights reserved. 


\title{
ABSTRACT OF THE DISSERTATION \\ MULTIDIMENSIONAL LEADERSHIP: MASCULINE AND FEMININE \\ LEADERSHIP APPROACHES IN PUBLIC EDUCATION
}

\author{
by
}

Joseph P. Eberhard

Florida International University, 2017

Miami, Florida

\section{Professor Peter J. Cistone, Major Professor}

With two-thirds of new leaders being women, it has become increasingly more clear that the face of our educational organizations is changing both symbolically and substantively. The demands placed on public education have also grown exponentially. If society demands that schools become better, then it is necessary to investigate the approaches that school leaders utilize in making decisions.

The purpose of this research was to explore the different approaches that educational leaders implement during their decision-making processes. The present study asked 20 school leaders within Miami Dade County Public Schools to report and explain their personal approaches to leadership to determine if these individuals utilize the reporting categories of masculine, feminine, or multidimensional decision making.

Following a four-round modified electronic Delphi technique, involving an openended questionnaire, a situation specific decision making survey, and two subsequent rounds of reflection, it was determined that the majority of school leaders use a multidimensional approach in making decisions; however, these individuals did not adhere strictly to any one of the specific reporting categories discussed. The study 
showed that the incorporation of several approaches contributed to the decision-making processes of educational leaders.

Although contrary to relevant literature in the field, participants' leadership approaches were not stringently tied to whether they happened to be male or female. The results of the current study suggest that research in sex-role orientations and gender studies, especially in the fields of organizational and educational leadership, may be evolving. The complex nature surrounding leadership in schools may imply that the infusion of a diverse or multidimensional approach to decision making is a necessary part of steering an organization towards a path that meets contemporary education demands.

The environment particular to each school determines the leadership approach that is appropriate. Environmental variables may include the school's history, the demographics of students and personnel, and details involving the type of organizational culture that has been fostered. Practical implications of the current study include exploring appropriate leadership and the role that multidimensional leadership approaches have in fulfilling the needs of specific schools. These leadership styles not only incorporate masculine and feminine leadership approaches, but also integrate approaches that can be considered androgynous and multidimensional. 


\section{TABLE OF CONTENTS}

CHAPTER

PAGE

I. INTRODUCTION

Statement of the Problem 3

Purpose of the Study

Statement of Research Questions 6

Significance of the Study 6

Delimitations 8

$\begin{array}{ll}\text { Operational Definitions } & 8\end{array}$

II. LITERATURE REVIEW 12

Management 13

Men as Managers 15

Women as Managers 17

Women Adopting New Approaches 19

Men Adopting New Approaches 21

Organizational Leadership $\quad 24$

Masculine Leadership 26

Feminine Leadership $\quad 30$

Dichotomous Extremes 34

Multidimensional Leadership $\quad 35$

III. METHODOLOGY 40

Research Questions $\quad 42$

Method and Process $\quad 43$

The Delphi Technique $\quad 43$

Why Modified Electronic Delphi? $\quad 44$

Procedures 45

$\begin{array}{ll}\text { Participants } & 51\end{array}$

IV. FINDINGS 54

Introduction to Results $\quad 54$

Reporting Categories $\quad 58$

Presentation of Findings $\quad 62$

Round One $\quad 63$

Round Two 68

Round Three $\quad 70$

Round Four $\quad 75$

V. DISCUSSION $\quad 82$

Summary of the Study $\quad 82$

Statement of the Problem $\quad 82$

Statement of the Procedures $\quad 83$

Statement of the Research Questions 83 
Summary of the Findings $\quad 84$

Conclusions $\quad 86$

$\begin{array}{lr}\text { Consistencies with the Literature } & 88\end{array}$

Inconsistencies in the Literature $\quad 92$

Limitations $\quad 93$

Implications for Research 101

Suggested Further Research 102

$\begin{array}{ll}\text { Summary } & 104\end{array}$

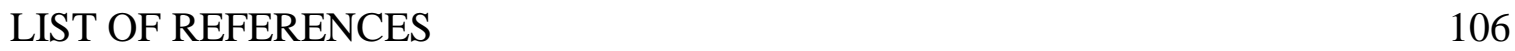

$\begin{array}{ll}\text { APPENDICES } & 108\end{array}$

$\begin{array}{ll}\text { VITA } & 145\end{array}$ 


\section{LIST OF FIGURES}

FIGURE

PAGE

1. Round One Overall 68

2. Round Two Overall 70

$\begin{array}{ll}\text { 3. Round Three Overall } & 75\end{array}$

4. Round Four Overall 80

5. Results by Reporting Category per Round 81

6. Participant 1 Results 112

7. Participant 2 Results 113

8. Participant 3 Results $\quad 114$

9. Participant 4 Results $\quad 115$

10. Participant 5 Results 116

11. Participant 6 Results 117

12. Participant 7 Results 118

13. Participant 8 Results 119

14. Participant 9 Results $\quad 120$

15. Participant 10 Results 121

16. Participant 11 Results 122

17. Participant 12 Results 123

18. Participant 13 Results 124

19. Participant 14 Results $\quad 125$

20. Participant 15 Results $\quad 126$

21. Participant 16 Results 127 
22. Participant 17 Results

23. Participant 18 Results

24. Participant 19 Results

25. Participant 20 Results 


\section{CHAPTER I}

\section{INTRODUCTION}

Today's K-12 public schools require adept leaders who are able to adapt to the diverse environments they traverse, as well as understand the impact of the particular leadership styles and approaches that they utilize. Research has been primarily concerned with linking productivity and efficiency with high-achieving schools (Jang, Ott, \& Shafritz, 2011). Productivity is contingent upon a results-based concentration or a mission that is outlined and established by an organization (Kruger, 2008). Gender studies offer an opportunity to observe heads of organizations as they navigate through the pressures placed on public schools. Organizational leaders operate by the use of both masculine and feminine leadership qualities (Coleman \& Pounder, 2002). Although the sex of the leader often plays a role in the perceived leadership attributes of the individual, the strength of the research should not rely on biological difference alone. More significant emphasis should be placed on the particular leadership style practiced by the individual.

As heads of institutions, leaders have been expected to possess the prowess of hero-like figures, both enthralling subordinates with the feats of their charge and demanding the respect of all those employed below them (Christman \& McClellan, 2012). A common theme in the literature is the hierarchical and/or top-down order of traditional leadership (Coleman \& Pounder, 2002). The aforementioned ideas illustrate a clear and concise depiction of a classical manager. The structure of such environments would reflect a pecking order gradation of both power and authority within the ruling ranks of an institution. The trickle down nature of order from superior to subordinate exemplifies both positive and negative aspects of the classical approach to management. 
According to Jang, Ott, and Shafritz (2011) the top-down approach maximizes efficiencies by allowing workers to increase their competence in accordance with specific tasks they are assigned. A division of labor with responsibilities completed by specific persons allows for an ongoing assembly-line approach to productivity (Jang et al., 2011). Specialization is achieved by these efforts leading to a productive work force. However, it is pertinent to note that organizational managers and their constituents are not simply mechanistic units. Classical management theory provides an opportunity to understand the leadership structure of school personnel (Lewin \& Regine, 2003).

Organizational leadership research has emerged as a relatively new form of study to understand what approaches of organizational management are appropriate for modern society. Leadership itself was once thought to go hand-in-hand with management; however, further research has unveiled it as its own unique phenomenon worthy of recognition and study (Sahin, 2011). Research in organizational leadership has uncovered a corollary relationship between management and leadership (Sahin, 2011). Effective organizational management cannot exist without in the same breath detailing leadership and the varying approaches and styles those leaders possess.

Schools are organizations (Sergovanni, 2009). The success of public education itself depends on the success of each school organization to deliver results. A growing list of demands on education has snowballed at an alarming rate. From federal legislation and litigation alike to state driven mandates, the local schoolhouse has been entrusted with responsibility to systematically improve teaching and learning (Gelberg, 2007).

Public schools also offer a reliable snapshot into current issues, themes, and solutions in contemporary management and organizational leadership research 
(Sergiovanni, 2009). A structure of influence and power like that of a spider web exists within educational leadership. The team of school administrators, academic department heads, curriculum support specialists, counselors, and district support personnel make up the many elements in education that develop and formulate decisions (Sergiovanni, 2009). These components are like that of a spider web. Each part contributes to the strength and success of the whole organization and in this case, to the effectiveness of a school. The leadership style and approach that these individuals utilize will ultimately determine the success or failure of an organization.

The present research intended to explore how current school leaders make decisions and determine what leadership styles and approaches they most often utilize. More specifically, the study applied supporting literature to recognize the approaches of current leaders within schools and whether their decision-making processes are masculine, feminine, or multidimensional. The intent was not only to unveil the attributes of actual educational leaders in the field but also to explore whether they implement a multidimensional leadership style that incorporates both masculine and feminine approaches. The current study ultimately looked at the decisions that school leaders make and determined whether masculine, feminine, or multidimensional approaches were used (Coleman \& Pounder, 2002).

\section{Statement of the Problem}

Educational leaders must embrace both masculine and feminine characteristics and use diverse approaches in the tackling of contemporary issues in education (Coleman \& 
Pounder, 2002). Individuals who adopt a multi-faceted style of leadership and management are better able to deliver a clear and concise vision to stakeholders (p. 124). According to Cheng (1994), every dimension of organizational leadership, including decision making can be categorized as a masculine or feminine approach. Additionally, each decision made by school leaders, whether masculine or feminine, has a consequence. There are individuals who exercise a masculine versus a feminine approach. However, the individual who is able to incorporate both masculine and feminine aspects of leadership will be the most appropriate manager for any particular organization (Riches \& Morgan, 1989). These leaders operate by sharing values and beliefs from both sides of the gender dichotomy, thus creating what Lewin and Regine (2003) claimed as "third possibility leadership" (p. 349).

With two-thirds of new leaders being women, it is becoming clearer that the face of our educational organizations is changing in both a symbolic and substantive way (Currie, 2007; Danielson \& Schulte, 2007; Chard, 2013; Naidoo \& Perumal, 2014). The K-12 education community relies on connections amongst things and people. There are various connections that are a vital part of the operation of public schools: curriculum to students, parents to schools, policy-makers to the citizenry, and even perhaps society with our collective ideals. Public schools are not only where children go to learn, but they are also where social justice emerges and takes center stage (Beachum \& McCray, 2010; Evans \& Reed, 2008; Reed, 2012; Sawyers, 2009). The relationship among sex, gender and leadership is a current theme in social justice that is a significant component of contemporary research. 
Coleman and Pounder (2002) contended that the arguing for one gendered leadership style over the other "misses the point" in defining effective leadership and management. It is rather the leadership approaches utilized and whether a fine balance between masculine and feminine is harnessed. The argument that surrounds this discourse is neither to determine whether it is better to be a man or woman as the leader of an organization, nor is not to bolster one gender and disparage another. The primary contention is to be aware of the evolving nature of contemporary organizations and to bring light to the essential need for our institutions and their leaders to evolve simultaneously.

\section{Purpose of the Study}

The purpose of this study was to identify and explore current school leadership decision-making approaches and whether they can be defined as masculine, feminine, or

multidimensional. Relevant research identifies masculine and feminine approaches as both distinct and significant aspects of effective organizational leadership. Additionally, the literature recognizes a multidimensional leadership as a possible way to accommodate the diverse needs within public schools. Ultimately, this dissertation explored leadership approaches and provides a foundation for future research that is needed to contribute to the body of literature surrounding organizational leadership. 


\section{Statement of Research Questions}

1) On the basis of a review of the literature, what approaches of current MiamiDade County Public School leaders may be understood to be masculine, feminine, or multidimensional?

2) On the basis of the literature concerning masculine, feminine, and multidimensional leadership approaches, given information about other leaders' responses, how do Miami-Dade County Public School leaders report and explain their leadership decisions?

\section{Significance of the Study}

Traditional viewpoints of effective management often point in the direction of male leaders who possess masculine characteristics (Ayman \& Korabik, 2010). In this case, leaders exhibit hero-like attributes. They are to be feared and respected as they lead their constituents into battle (Kruger, 2008). A power-over structure is put into place, which emphasizes heavily traditional or classical forms of management (Lewin \& Regine, 2003). These play heavily on the discipline and productivity aspect of organizational structure. Ayman and Korabik (2010) developed a picture of what stereotypes are correlated with masculine management: aggressive, assertive, and impersonal. Despite the fact that the majority of public school employees are women, these organizations are predominately run by men (Coleman \& Pounder 2002).

Research has indicated that there are positive attributes to both masculine and feminine leadership. Feminine leaders tend to be collaborative, empathetic, and focus on the importance of individuals (Ayman \& Korabik, 2010). Coleman and Pounder (2002) 
argued that in order to practice effective leadership, the strengths of both genders should be embraced. Incorporating both masculine and feminine attributes allows for a more diverse approach in the tackling of contemporary issues in education (Coleman \& Pounder, 2002). The individual who adopts a multi-faceted style of leadership and management will be better able to deliver a clear and concise vision for stakeholders ( $\mathrm{p}$. 124). Utilizing both the approach of persistence, which is recognized as masculine and cooperativeness, which is identified by the research as feminine is persuasive and may lead to multidimensional leaders being better able to bargain for greater social-capital within organizations. Coleman and Pounder (2002) claimed that multidimensional leaders are best suited to deal with both internal and external pressures on organizations.

According to Cheng (1994), multidimensional leadership constitutes a mixture of masculine and feminine. It is the individual who effectively incorporates both aspects of a masculine and feminine leadership paradigm, who will be the most appropriate leader for a particular organization (Riches \& Morgan, 1989). These leaders operate by sharing values and beliefs from both sides of the gender dichotomy, thus creating what Lewin and Regine (2003) called "third possibility leadership" (p.349).

Third possibility leaders value diversity and communication, while simultaneously emphasize being assertive and analytical. Both men and women can be third possibility leaders, because being third possibility refers to going beyond gender. Coleman and Pounder (2002) agree that being genderless or "exhibiting androgynous leadership style" is a key point in understanding effective organizational leadership and management (p.122). It is necessary that educational leaders embrace practices that improve their capacities to make decisions that are appropriate for the organizations in which they work. 


\section{Delimitations}

The sample size of 20 selected for this study will include the voluntary participation of K-12 public school leaders from the large urban school district of MiamiDade County Public Schools. The participants sought for this study will include school principals, assistant principals, instructional coaches, district support personnel, community involvement specialists, team-leaders, counselors, and department chairpersons. The sample size used in the mixed methods procedures may limit the current study's external validity; however, the findings will be strengthened by further research that is detailed in the discussion chapter.

\section{Operational Definitions}

Androgynous Leadership. These individuals do not convey a one gender-type approach, but rather adopt what researchers are calling "androgynous" leadership (Ayman \& Korabik, 2010, p. 165). This term was used to determine the multidimensional style leadership of participants as measured by their answers on questionnaires, surveys and other various responses during the course of this study.

Contemporary Society. Specific current era facing schools with an emphasis on highstakes testing, data driven instruction, and the role of that the public school house in both educating students and accommodating the demands of society (Beebe \& Nogay, 2008;

Christman \& McClellan, 2012; Sergiovanni, 2009) 
Leadership. Leadership is influence (Northouse 2013). It relies on of implementation of a set vision and mission and how well stakeholders reach the established goals of the organization (Ayman \& Korbik, 2010).

Management. An emphasis on top-down relationships with superiors and subordinates. This approach to running organizations often depends on roles and responsibilities (Jang, et al., 2011).

Multidimensional Leaders. A type of leadership that blends masculine and feminine approaches that is necessary to the needs of the organization (Coleman \& Ponder, 2002). This term will be used to determine the leadership style of participants as measured by their answers on questionnaires, surveys and other various responses during the course of this study.

Positive School Culture. The thoughts, actions, and efforts of the school as a whole reach for student achievement collaboratively and are succinct and in step with the leadership practiced (Segiovanni, 2009).

Self-Efficacy. Self-efficacy relates to a belief system in which an individual believes that he/she is capable of performing a specific task. While it involves "perceptions of one's capabilities" it is not the expectation of outcomes (Schunk, 2012, p. 146). 
Self-Monitoring. Self-monitoring requires that individuals take careful observation of the results of their behaviors. (Schunk, 2012, p. 401)

Self-Regulation. The process of identifying best practices and measures to focus and better one's self. (Ayman \& Korabik, 2010).

Third Possibility Leaders. These leaders operate by sharing values and beliefs from both sides of the gender dichotomy, thus creating what Lewin and Regine (2003) as "third possibility leadership" (p.349). This term will be used to determine the leadership style of participants as measured by their answers on questionnaires, surveys and other various responses during the course of this study (Lewin \& Regine, 2003).

Titular Leadership. Title defines the authority and responsibility of an individual or individuals. (Coleman \& Pounder, 2002; Northouse, 2013).

Traditional Leadership (Theory). A "hierarchical" and/or "top-down" order of organization power and influence (Coleman \& Pounder, 2002).

Traditional Management (Theory). A top-down managerial style that emphasizes taskdriven initiatives. (Taleb, 2010).

Transactional Leadership. A focus on "goals, roles, and responsibilities" (Reynolds, White, Brayman \& Moore, 2008, p.23) 
Transformational Leadership. This blurs the distinction between formal and informal, and asks both the leader and follower to grow together beyond expectations (Murphy, 2005). Utilizing the "ethic of care, connectedness, and sort of participative style" this approach offers an influential method of leading public schools (Oplatka, 2006, p. 606). 


\section{CHAPTER II}

\section{LITERATURE REVIEW}

The purpose of this study was to identify and explore current school leadership decision-making approaches and whether they can be defined as masculine, feminine, or multidimensional. The review of the literature will present the evolution of management and organizational leadership theory as a foundation for further research and discussion. Providing this roadmap gives the reader a direct-line of understanding into how the styles of organizational leaders have adapted to better suit the needs of the present environment. Furthermore, this review of the literature establishes a divide between observable styles of masculine and feminine leaders. It is imperative to establish that descriptions of masculine or feminine involve gender, but this is not an indication of the sex of an individual. The following will further clarify this concept.

The differences between masculine and feminine are central to this discussion and will allow for an observation of gender-specific approaches and their impact on organizations. Additionally, this research conveys that leaders are being asked to utilize a multidimensional style, where approaches are adopted independent of either side of the gender dichotomy to accommodate the demands of contemporary society. Relevant literature will illustrate the relationship between the type of leadership approach that is practiced and the needs of schools. By following the evolution of organizational leadership in coincidence with the perceived influences of leadership approaches on stakeholders, this study will be better able to identify and develop consensus on what constitutes appropriate leadership (Guramatunhu-Mudiwa \& Bolt, 2012). Chapter II is organized by presenting and describing the relevant themes that exist in the literature. 
The first area explored is the aspect of organizational management, its history, and how it impacts current school leaders. The review of the literature will be primarily concerned with identifying the different styles of management. Chapter II will draw on the research to define approaches that are different and how leadership style may differ in regards to gender and decision making. The following sections are concerned with leadership and how masculine and feminine leaders operate in organizations. Again, drawing upon distinctions, the research will reflect the impact of these gender-associated approaches. The literature will show that appropriate leadership is measured by a mixed approach. This chapter presents a mixed approach as multidimensional leadership.

\section{Management}

Organizational management involves more than simply overseeing and directing personnel. It requires a broad perspective with the ability to see a much broader picture than what currently seems relevant. Organizations that adapt and prepare are more able be successful in the complexity of today's contemporary society (Ayman \& Korbik, 2010). Managers are not only required to have the ability to keep a larger perspective, but also have the capacity to narrow their focus. A narrow focus allows for clarity, specific task completion, and successful implementation of initiatives. Clarity is essential in the operation of successful organizations, which contributes to more effective communication with intent and beckons necessary follow-through. One of the greatest challenges of organizational management is ensuring a culture of follow-up and extinguishing areas of negligence in task completion (Lewin \& Regine, 2003). To overcome this challenge, 
managers must be able to understand relationships and collaborate with people key individuals within their organizations.

One cannot speak of management without including the supervising of subordinates into the determination of efficiency and effectiveness. Organizational management requires that job descriptions are clear and that the top-level managers and supervisors can deliver specific directives. They must see the picture of how each employee fits into the greater scheme and ultimately works towards the vision and mission of that organization (Ford, 2005). The emphasis is not on interpersonal knowledge of personnel, but rather on the responsibilities that particular job titles and positions require. Managers, therefore, operate with a set of clear expectations and hold employees to these expectations. The lack of concern for the interpersonal and knowledge of intimate details about personnel may be a factor leading to observed chaos in the complexity of today's contemporary society (Taleb, 2010). Organizational management may incorporate an emphasis on the roles and responsibilities of individuals within the whole; however, it fails to take into consideration how to understand individuals and their needs.

Successful organizations inevitably have a management that is considered effective with both a narrowing focus and an ability to know, understand, and serve the personnel (Ford, 2005). Careful consideration into these areas aims at the facilitation of a positive culture with consistent improvement and success (Marinescu, Saseau, \& Toma, 2014). From the ever-changing demographics of the citizenry of the United States, the way communication is sent and received, and the availability and access to infinite information has resulted in a tumultuous environment (Barnett \& McCormick, 2004). These turbulent times have contributed to the increasingly complex nature of today's society. The review 
of the literature does not intend to declare these changes as good or bad, but it rather establishes the current state of society as a variable in unraveling perceptions of what constitutes organizational management. In order to know, embrace, and overcome these challenges, understanding the historical perspective of management is an inherent need. The literature focuses on presenting the characteristics of different managers through the lens of whether they are male or female. The following sections will utilize this framework to describe different styles of managers.

\section{Men as Managers}

Coleman and Pounder (2002) claimed that men tend to be authoritarian in their approach. Authoritarian manager's words and actions stem from a central emphasis of roles and responsibilities. These duties and obligations keep the system running, and the men that run these organizations lean on their subordinate managers to communicate expectations. Because the top-level management may never interact with the lowest level employee, their relationships tend to be focused on superior to subordinate, similar to a top-down approach. This structure creates a system of accountability, where every individual is responsible to someone who is either above or below him or her in the chain of command. Men as mangers bolster productivity through rewarding hard work and employees that put forth extra effort and reinforce negative actions and behaviors with sanctions. Organizations run by men emphasize jobs and duties as measured by on taskcompletion (Owen, 2007).

The emphasis on roles and responsibilities within an organization is undoubtedly an intricate part of effective management. Organizations managed by men emphasize the 
importance of roles in a hierarchical structure (McCormick, 2004). Despite one position being above another, the importance of each individual or team of persons fulfilling the required task is essential for success (Goodman \& Zimmerman, 2000). Similarly to classical management theory, the well-oiled parts of the machine result in better function and a better end product (Riches \& Morgan, 1989). As with an assembly line, one break in the communication, one individual not fulfilling their role, or one group of people not working up to their ultimate capacities, the overall result ends in less efficiency and productivity. Men as managers focus on these roles and responsibilities and use this emphasis to empower superiors surveying and observing the activities within an organization (McCormick, 2004; Owen, 2007). This oversight is essential for the operation of a well-oiled machine and portrays a top-down hierarchical structure.

In order for oversight to take place, men as managers oversee the production within the organization by establishing a system of superiors and subordinates. Each of the supervisors checks and ensures that each part of the whole is fulfilling the expectation set forth by top-level management. The structure in these organizations resembles and chain where the top-level management is the central point and each link stretches out from this center support. Each line is a section tied to a supervisor which intern is stretched out to many more strands symbolizing the many working parts of an organization (Burns, 1978). The chain is only breaks at the weakest strand or connecting point; therefore, men as managers emphasize a top-down roles and responsibility focused system that provide reinforces the form of rewards or sanctions.

Men as managers lean heavily on the job description details and depend on its contents to hold personnel accountable for their work (Coleman \& Pounder, 2002). 
Whether this is not reaching productivity expectations, or whether deliberately not fulfilling the obligations of employment, the literature states that men view their employees through a finite lens (McCormick, 2004; Owen, 2007). They do not take into consideration the person, but rather the job that needs to take place. Sanctions used to redirect and hopefully support a positive turn around include verbal and written reprimands, loss of hours or wages, reassignment, leave without pay, suspension, and ultimately loss of employment (Owen, 2007). Even though the literature ties men that are managers to a list of leadership approaches, the question is does masculine leadership really correlate to managers that are men? First, let us look at women as mangers and determine what their differing operational approaches offer.

\section{Women as Managers}

The study of women as top-level managers unveils organizations that operate with contrasting approaches when compared to that of their male counterparts (Vladero, 2009). These organizations reach for higher efficiency and greater productivity in a distinct manner. Women view a structure of an organization, its personnel, and how tasks should be completed within a holistic mindset (Taleb, 2010). They also do not typically view management from a top-down approach, but rather look at people in terms of teams. These groups collaborate to work towards task completion, rather than focusing on individual

effort. Instead of an emphasis on the weakest link, women focus on productivity of the team (Goodman \& Zimmerman, 2000). Women also establish a culture that provides incentives in a diverse way (Lewin \& Regine, 2003). Through validation and an emphasis 
on relationships, women tend to incorporate a political aspect to power structures in organizational management.

In contrast to a focus on roles and responsibilities, where the organization's success is tied only to the output of each person, women support coalition building (Vladero, 2009). Kloot (2004) argued that one differing factor for women as managers is their "concern for people and relationships" (Kloot, 2004, p. 479). It is not one person who keeps the machine well oiled, but a team of people who ensure the success of the whole. It is through the efforts of many that tasks are pinpointed and completed. It is not simply the focus on teams, but more so of the collaboration that exists between and amongst members of the group (Donaldson, 2006; Sahin, 2011). Therefore, women as managers support the forming of relationships amongst people within the organization.

These relationships are what lead to effective communication, a healthy work environment, and ultimately cohesive productivity. Lewin and Regine (2003) concluded that "relationships [are] the bottom line for business success in this complex environment" (Lewin \& Regine, 2003, p. 347). Women managers care about the individual rather than the person as an employee only. Through the recognition of the person, women follow a more neo-conservative viewpoint of organizations, where the only way to increase efficiency is through improving communication within the organization (Moreno \& Yanez, 2007). The emphasis on organizational socialization contrasts the top-down approach as women support power within rather than power over. In other words, an organization's success is not determined by the greatness of the top manager, but rather by the strength of the group together. 
Organizations managed by men signify a chain of command, where women support communities of interconnected webbing. This web is not easily broken, but unraveling exists through the inability of each team or strand to communicate, collaborate, and complete tasks together. Women as managers reinforce positive actions and behaviors through ostracizing those who do not assimilate into the culture of the organization (Kruger, 2008).

Women look for teams and groups to identify as examples and models for the organization to work toward, attain, and make commonplace across the board. Additionally, women managers highlight the strengths of the individual, which has a profound impact on the culture of the organization (Johnson, 2012). Treating employees from a holistic viewpoint leads to an organization that is tied together by definition. This commitment in turn improves communication, and develops a sense of belonging through community. The organization is no longer simply a place of work, but a meaningful community that takes into account the employees as individuals and how they interact (Jang et al., 2011). Despite the different perspectives that women as managers offer, there is a muddying of the distinctions between male and female leaders. The following sections will present both women and men adopting new approaches to be better managers for their respective institutions.

\section{Women Adopting New Approaches}

The literature contends that leaders who are women find it necessary to adopt the characteristics of men to receive respect as managers (Kloot, 2004). It is because of a fear of losing the respect of peers and constituents that women are persuaded to adopt these 
new characteristics. A study conducted by Anderson and Klofstad (2012) found that "females with masculine voices" are perceived as being more effective as heads of organizations (Anderson \& Klofstad, 2012, p. 2). Their research has simply highlighted what viewpoints have impermeably entrenched our views of what leadership must constitute. Moreno and Yanez (2007) argued that this has caused the "impregnating" of masculinity into what society perceives of leaders. The concept that top-level management must possess masculine qualities has become an inherent part of our understanding of organizations. The masculine imprint on the excepted understanding management has undoubtedly influenced women and those who strive to become managers themselves.

Women in many cases adopt these practices as they emphasize roles and responsibilities, institute top-down hierarchical relationships, and reinforce loyalty to particular visions through reward and sanction (Kloot, 2004). Kloot (2004) argued that women adopt these traits out of necessity to be perceived by their constituents as competent leaders. These individuals may even adopt a complete male-dominated approach, either out of fear or not wishing to be seen as weak. The research suggests that these women often bring into question the authenticity of their management, as they are defying stereotypes (Calvanese, 2007). A study conducted by Brown and Light (2012) found that women were willing to sacrifice their typical collaborative approach for the trait of making rational pragmatic decisions (Brown \& Light, 2012).

The pressure to perform and be successful has inhibited women from empowering themselves as unique and diverse mangers that offer a contemporary answer to the complexities in today's society (Kloot, 2004). Women attempt to counter-balance fears of being perceived as weak, or not as effective when compared to their male counter-parts 
sometimes adopting an extremely different approach that is more masculine (Ayman \& Korabik, 2010; Reynolds et al., 2008; Moreno \& Yanez, 2007; Lewin \& Regine, 2003). This, too, has perceivable risks in the likelihood of being perceived by stakeholders as inauthentic.

The conversation, however, is evolving to include how women have been able to incorporate the most appropriate approach and style into their leadership decisions dependent on the environments in which they lead. The literature recognizes the influence of longstanding masculine lens that has been used to view proper leadership attributes. More specifically, the discussion has been shaped by these styles that have been typical of men, causing women to implement their own unique styles of leadership (Ford, 2005, p. 241). In many cases, their approaches incorporate both masculine and feminine qualities, which would seem to offer the best of both sexes.

\section{Men Adopting New Approaches}

Sahin (2011) suggested that the way to improve the organizational culture within schools, men must incorporate both masculine and feminine qualities into their everyday leadership practice. This involves collaborating with the stakeholders of a school to create a common vision and mission, to develop unity of action, and to share in the action of leadership (Waters \& Cameron, 2007). The emphasis on communication is recognized as a feminine trait that leads to the creation of a "culture of progress" (Sahin, 2011, p. 1924). Men have an opportunity to accommodate the needs of today's complex society by incorporating masculine and feminine qualities and learning to apply the appropriate leadership style that is necessary for the setting and the community. Because "effective 
management requires a balance of feminine and masculine skills" these leaders must be all-encompassing (Lewin \& Regine, 2003) in thought and action (Coleman \& Pounder, 2002, p. 128).

Lewin and Regine (2003) offered a snapshot for what they called third possibility leadership. These individuals represent paradoxical, resource gathering, "wholistic," and adept leaders who operate with relational intelligence (Lewin \& Regine, 2003). The nature of an emerging third possibility leader that is not entirely masculine or feminine. His/her androgynous style is a contradiction in itself where he/she can be "tough and empathetic... patient and timely... competitive and collaborative" (p. 349). The paradox is that a third possibility leader can be strong through soft ways. Ultimately, school leaders with innovative styles bring people together.

There are men who refuse to adopt new traits, which leads to the creation of an environment that is described as toxic or hyper-masculine (Kruger, 2008). These individuals seem to operate with a blatant disregard for the needs of the community and what is required for progress of the organization. It is not dissimilar to a racehorse wearing blinders. The organizational leader is racing towards the finish line, which in the case of a school is the end-of-year or the actual date of the annual assessment, without accepting the input of others. The indifference to changing circumstances and needs of particular settings prevents the racehorse from adapting to unforeseeable events or changing its stride to better lead throughout instances of adversity (Moreno \& Yanez, 2007). Leaders that are men are turning away from the top-down managerial approach that used to dominate many aspects of traditional leadership and are now becoming more third possibility (Ayman \& Korabik, 2010; Coleman \& Pounder, 2002; Lewin \& Regine, 2003). 
Lewin and Regine (2003) called these individuals "gatherers" who communicate effectively not by overpowering stakeholders in a school, but rather by bringing functionality to dysfunction. They inspire, not just motivate personnel. Third possibility leaders understand the nature of power in an organization and they use it to bring "nourishment not fear" (Lewin \& Regine, 2003, p. 350). Actions of top-level management represent a form of symbolism. For example, these individuals know that they are role models and that their actions can be amplified to represent the entire organization. A third possibility leader ties actions to decisions that benefit their organizations by making connections between things and people.

Strong leaders are great communicators. They bring people together and do so by aligning their efforts toward commonalities. In a school, principals influence each level of leadership to solicit the buy-in from all parties so that collective unity is reached. Relational intelligence plays to the inherent social nature of human beings. This socialization conveys as a better with, rather than a better without mentality, which empowers personnel and creates a culture of compassion not confrontation, unity not exclusion, and trust not skepticism (Lewin \& Regine, 2003, p. 350). It is not enough that men become more feminine in their approach or that women adopt masculine styles of leadership, there must be a mindful and deliberate approach to school leadership.

Lewin and Regine (2003) conceded that unless women exhibit some traditional or masculine qualities of leadership, then they will not be successful (Lewin \& Regine, 2003). The same can now be assumed for men as we have seen that masculine leadership in its extreme leads to an alienated staff and faculty and possibly the excommunication of the top-level manager. As society evolves and our ideas of organizational leadership along 
with it, the "feminine manner can be the key to success in enhancing the competitiveness of an organization” (Marinescu et al., 2014, p. 147). In a school, this translates to higher student achievement and greater school success.

The review of the literature suggests that this new leader is not to ignore what leadership style comes natural, but rather be aware of how his/her leadership approach may be perceived and how it may influence constituents within an organization. Furthermore, the demand to understand the "independence between masculine and feminine" must be taken into consideration when honing one's leadership skills (Marinescu et al., 2014, p. 148). As our ideas of top-level managers and school leaders evolve, those individuals entrusted with the task of raising student achievement and bolstering the effectiveness of schools, are obligated to improve their craft. This involves taking specific steps to improve one's leadership capacity. The literature suggests that the pathway to guiding schools towards greater student achievement is through multidimensional and third possibility leadership (Ayman \& Korabik, 2010; Coleman \& Pounder, 2002; Lewin \& Regine, 2003).

\section{Organizational Leadership}

Although not all generalizations and stereotypes apply in every circumstance, the research identifies and distinguishes between specifically the terminology of men and women as leaders. Referred to as sex-role stereotypes, Eckman (2004) stated that there are perceivable discrepancies between the two sexes. Beebe and Nogay (1997) called these differences significant. Women principals were effective at communicating their visions, while men had strengths in supervising and evaluating (Beebe \& Nogay, 1997). The 
contrasting examples between males and females can be attributed to both biological and environmental factors that impact decision-making (Eckman, 2004).

Kruger (2008) argued that the ability of men to grasp a greater spatial understanding and women that are better at communication is a result of neurological connections not "gender role socialization" (Kruger, 2008, p .157). These differences influence managers and leaders, and ultimately have bearing on the vision and mission of the organization, the actions taken to accomplish common goals, and the overall culture supported in the company or institution (Waters \& Cameron, 2007). In public schools, the actions of leaders affect the culture by creating environments that are conducive to collaboration and invite the input of the stakeholders (Currie, 2007). There are organizational cultures that are concerned with command and control and operate similarly to the classical managerial theories explored earlier. These school leaders are influenced through the powers of titular leadership, which is concerned with title and position.

Appropriate leadership is the ultimate goal, where the approaches or styles of masculine and feminine leaders lend themselves to particular strengths and weaknesses. Often, it is the environment, culture, and history that determines the type of leadership required. A school may benefit from the influence of masculine leader, where feminine leadership would not be as effective. The inverse is also a possibility, which again is determined by the details of a particular organization. Neither masculine nor feminine leadership can be universally revered over the other. This discussion offers observations through a masculine and feminine leadership paradigm. When an individual operates at 
one extreme of the paradigm or the other it illustrates either hyper-masculinity or hyperfemininity (Chistman \& McClellan, 2012).

Both extremes are an overemphasis of one leadership approach over the other. Each is viewed as dangerous and toxic to organizations (Lewin \& Regine, 2003). These toxic leaders poison cultures and as a result create both a lack of effectiveness and a decrease in productivity. Individuals who practice one of the extremes often find themselves as ineffective leaders who are pushed out and eventually ousted from their positions.

\section{Masculine Leadership}

Masculine leaders orchestrate leadership in comparable terms to classical management. Because the majority of managers have historically been men in Western cultures, our societal views of what constitutes leadership have been imprinted with certain expectations that are aligned with a masculine and often a male-dominated point of view (Ford, 2005). Traits that emphasize strength and power over a group of individuals are often described when depicting the traits of leaders. The ability of a person or persons responsible for leading an organization to streamline efforts towards common goals by being revered and respected are also important aspects of what have shaped our views of leadership (Polatka, 2004). The same is also true for school leaders.

Principals are the ultimate top-level leaders at K-12 school-sites. Throughout history in the western-world, men have fulfilled these roles and established a standard and expectation for school leadership (Taleb 2010; Vladero, 2009). The perception that roles such as superintendent or principal "[have] to be a man" is impregnated on both traditional 
and contemporary thought (Currie, 2007, p. 6). While the landscape surrounding educational leadership is changing, there are certainly more roles other than superintendent and school-site principals to take into consideration when seeking to understand those who make decisions. Other leaders in the school setting include assistant principals, department chairpersons, team leaders, and a large variety of stakeholders that contribute to decision making. The broad spectrum of leaders has grown as a result of the contemporary demands placed on public education. These individuals are often ambitious persons who have a passion for instituting change and facilitating the process of raising student achievement (Sahin, 2011). The spread of leadership responsibility has not always been shared among many stakeholders, which history has shown us has typically been limited to positions of status and title.

Leadership most likely includes a small ring of people such as the principal and assistant principal(s). Titular leadership, which power and authority based on position, title, and/or status, is a quality of organizations led by masculine leaders. Masculine leaders orchestrate their organizations with an emphasis on hierarchies. Owen (2007) has claimed that this results in a distinct style of management personnel. For example, employees are not looked at as specific individuals, but rather weighed as a human resource (Owen, 2007). Because sexual stereotypes no longer hold complete relevancy, both men and women can incorporate these masculine approaches in their decision making.

Masculine leadership has shaped the contemporary consciousness of leadership. School leaders who utilize this type of leadership operate comparably to classical management theory. Masculine leadership is concerned with an approach incorporating an 
unwavering persistence to task completion and loyalty to decisions made by institutional leaders (Coleman \& Pounder, 2002; Marinescu et al., 2014). The ultimate vision and mission is delivered from the top-down, and these types of leaders will focus on the actual task and the position of subordinates to delineate responsibilities. In schools, the emphasis is on school improvement through a Type-A personality culture and atmosphere (Christman \& McClellan, 2012). This carries through to every activity in which school leaders will enforce norms and established procedures to complete assigned tasks (Taleb, 2010). Each stakeholder is required to fulfill his/her area of duty.

The leaders implement a rigid structure in order to obtain compliance. A study conducted by Marinescu, Saseanu, and Toma (2014) found that motivating employees was not a primary objective of organizations when men were the managers (Marinescu et al., 2014, p. 147). Instead, they used excessive control to enforce initiatives. This can be seen as a toxic characteristic of excessive masculine leadership or hyper-masculinity (Lewin \& Regine, 2003). This type of leadership is also similar to classical management theory, where masculine leaders focus on roles and responsibilities. In order to increase productivity and ensure the success of the whole, these leaders look to each employee to ensure that obligations are being fulfilled. Masculine leaders also emphasize expectations through as system of accountability and follow-through.

Accountability is enforced in schools through several factors with an emphasis on student performance results. Performance is tracked annually and the purpose is to serve as a snapshot of a school's perceived success. Masculine leaders also emphasize a datadriven decision making culture produced through this process to motivate individuals and push towards more effective teaching and learning (Owen, 2007, p. 6). School leaders will 
persuade stakeholders to work towards improvement by aligning their efforts to the established expectations through a loyalty to the institution's vision. Because interactions between school leaders and their subordinates are particularly concerned with task completion, roles and responsibilities, and expectations through accountability, most leadership decisions can be classified as transactional.

Relationships are an important part of leadership within organizations (Sergiovanni, 2009). Masculine leaders tend to act in ways that are considered transactional in nature, which are actions primarily concerned with delivering edicts and requiring a specific response to directives (McCormick, 2004). Therefore, relationships do not take into account personal strengths or abilities of employees and they certainly do not empower people to engage in a transformational process. Chistman and McClellan (2012) stated that if a leader is able to organize and utilize the beneficial forms of micro and macro-management, then the school will ultimately be successful. The power is within the hands of the school stakeholders and decision makers led by the principal. Accountability rests in his/her hands and they are entrusted with the authority to have the appropriate conversations and transactions with employees within the school to ensure that the organization is on a pathway towards greater performance results. These highlighted approaches of masculine leaders have both positive and negative connotations. On the opposite end of the paradigm sits and diverse set of decision making styles more associated with feminine leadership. 


\section{Feminine Leadership}

Feminine leaders have an alternate perspective of what constitutes appropriate leadership. The literature has developed a body of approaches that have been described and can be identified as feminine. Impacted by both biological and environmental factors, these new and distinct perspectives have offered an innovative approach to organizational leadership. One such consideration is the emphasis on socialization amongst individuals and the incorporation of collaborative efforts to complete tasks (Ayman \& Korabik, 2010; Kruger, 2008; Taleb, 2010). Women have also been more likely to be empathetic towards members of their community, showing consideration for the needs of individuals (Kruger, 2008). Although women have typically been described with utilizing these particular characteristics, both men and women are now implementing feminine leadership approaches. This type of leadership involves considering following uncommon pathways towards organizational success through a shared leadership model.

Shared leadership allows for the heads of organizations to spread responsibilities and enforce accountability not just by focusing on the positions and titles, but rather by empowering groups of people (Owen, 2007). The teams or circles of influence that are utilized under shared leadership are avenues for which directives and tasks are completed. By sharing responsibility in this manner, buy-in from personnel is increased. This aspect of feminine leadership is observable in schools where principals and assistant principals designate team leaders, department chairpersons, etc. with delegated power, and the authority to increase the productivity of the organization.

Productivity in education is measured by the ability of the school to increase student achievement and reach set goals established at the beginning of the school year. 
Establishing set goals have typically come in the form of the School Improvement Plan (SIP), which is a public document that sets the expectations for the school year. Feminine leadership does not limit the authors and contributors to this important document to a few, but rather entrusts teams of people to develop achievable expectations. The importance of each layer of influence within the organization becomes more evident, which emphasizes the need for leaders to devote resources and time to staff development (Lance, 2010).

Feminine leaders put increased stock into fostering the capacity of staff, which is observable in K-12 schools through more in-service training and professional development (Brown \& Light, 2012; Owen, 2007). Lance (2010) claimed that without the increased abilities of each part of the whole, the teams of personnel would be unable to complete tasks with urgency and efficiency. Lance (2010) later argued that staff development is a "critical" part of effective schools (p. 118). Greater effectiveness comes from a "people-oriented approach" through an investment in personnel, and employees reap the benefit of both increased capacity and the organization ultimately reaching set goals (Marinescu et al., 2014, p. 146). The ability of schools in these instances results in better faculties, staffs, and administrators. The emphasis on role is decreased and the focus is then redirected towards the nurturing of stakeholders to take initiative for making improvements.

Nurturing is an important part of successful organizations and is a vital part to the empowerment of personnel (Reynolds et al. 2008). This attention has allowed individuals like teachers to develop better instructional approaches, administrators to increase the ability to support teams of employees, and fosters the involvement from stakeholders. With greater input from a wide range of individuals, feminine leaders depend on the skills 
and expertise of every individual. Feminine leaders who emphasize the strengths of each person believe that the whole organization will benefit from increased capacities institution-wide (Brown \& Light, 2012). Organizations that value the input from each individual give reverence for both the personal and professional desires of stakeholders. Believing in individuals has allowed for leaders to not only know members of the team, but also serves their ability to emphasize dedication and buy-in for the established vision and mission. The empowerment of stakeholders allows for individuals that utilize a feminine leadership style to gain the respect from members of the organization resulting in greater commitment.

Increased commitment is fostered through feminine leaders' efforts in caring for individuals. The Ethic of Care is vital to the empowerment of personnel by operating in a supportive role. Leaders facilitate the growth process, by listening, providing feedback, and offering opportunities for growth (Brown \& Light, 2012; Eckman, 2004). The pressure to accomplish set goals influences members of organizations. Increase pressure does not ultimately entail a downfall, but does imply that some people may need special attention to ensure confidence and dedication. Self-efficacy is not an inherent quality of employees (Lance, 2010; Leithwood \& Jantzi, 2008). Improving this attribute must be fostered, which ultimately impacts the ability of the organization to be successful (Eilers \& Camacho, 2007). Listening to concerns of the stakeholders within a particular school allows for the leader to best accommodate and provide constructive feedback. Selfefficacious leaders understand the strengths and weakness of personnel. Providing appropriate feedback is an essential part of growth and a significant part of the this approach termed Care (Brown \& Light, 2012). 
Facilitating the growth process for employees allows for the heads of schools to guide the progression of development. Feedback comes in many forms, where the personnel are able to personally identify with set expectations. Relating directly with the institution's vision and mission allows the leadership to redirect efforts and align actions towards the successful completion of common goals. Owen (2007) argued that feminine leaders emphasize attention to the individual. These leaders are more "symbolic" and tend to have an impact on organizational "culture" (Owen, 2007, p. 7). Attention to the individual is an aspect of the Ethic of Care. Feminine leaders invest and support the development of the individual. These interactions are extremely important in organizations run by leaders who utilize a feminine approach.

Relationships often determine the level of buy-in and the overall cultural of the organization. To foster a positive environment, feminine leaders utilize transformational leadership (Brown \& Light, 2012; Taleb, 2010). School leaders look for opportunities to transform stakeholders to adapt and embrace challenges. These outlined practices of feminine leadership are viewed more as a bottom-up rather than a top-down approach to improving schools (Lance, 2010). Transformational leaders also facilitate organizations that are democratic (Brown \& Light, 2012).

Feminine leadership demands the input from every level and often each individual within a company or organization. Taleb (2010) advocated for this approach claiming that the heads of organizations are charismatic and "serve as role models" (Taleb, 2010, p. 296). The researcher also identifies feminine leaders as more holistically democratic in their solicitation of stakeholders for input in decision-making processes (Taleb, 2010, p. 290). 


\section{Dichotomous Extremes}

Both masculine and feminine leaders have strengths and weaknesses in their respective styles. Without proper implementation of an appropriate approach to the needs of the educational community, the effectiveness of the heads of schools comes into question (Shum \& Cheng, 1997). This can result in the loss of confidence and trust from constituents and the alienation of certain stakeholders. The greatest risk is the poisoning of the culture, which the extremes of each leadership style tends to do. School leaders must continue to seek feedback from members of the communities that they represent and ensure that they operate under a standard of authenticity.

Authentic leaders act following genuine principles that include a desire to move the organization towards good, rather than just striving for the good of self (Lewin \& Regine, 2003). School leaders reset their sights on student achievement and proclaim the need for each member of the organization to mimic this course of action. Asking teachers to commit themselves to be more self-reflective, follow evidence-based practices, and contribute to a culture of adaptive change is a daunting task for school leadership (Maslow, 1991). Leaders without the political clout within their organizations to demand or even ask stakeholders to sacrifice and face adversity cannot be done without being authentically tied to the established vision and mission (Kirst \& Wirt, 2009; Lance, 2010; Lewin \& Regine, 2003;). Both extremes within masculine and feminine leadership present decision making from a point of indifference. These are unable to take into consideration the dire needs and desires of the organization. These types of leaders have a difficult time building teams and collaborative approaches towards sustained student achievement

(Owen, 2007). 


\section{Multidimensional Leadership}

There is no "one size fits all" leadership style for public schools (Reynolds et al., 2008, p. 45). The complex challenges demanded of the K-12 education system require a multidimensional leader with a multidimensional approach. Kruger (2008) argued that school leadership is "being asked to do the impossible" (p. 156). Organizational leaders are obligated to understand how differing approaches of both masculine and feminine leadership might influence their roles. Reynolds, White, Brayman, and Moore (2008) argued that a key to effective decision making within companies and institutions is not only to be aware of these factors, but also to "balance gender on leadership teams" (Reynolds et al., 2008, p. 45). This diverse approach involves the supervision of positions by structure and action.

Taking from the strengths of feminine leadership, school administrators and team leaders should be collaborative, team oriented, and open communicators. Additionally leadership should reflect a value in relationships in their actions and also serve as role models by "leading by example" (Brown \& Light, 2012, p. 189). These feminine qualities should be expressed throughout the leadership team, whether the individuals holding the positions are male or female. The demand is simply that awareness of the effect of sex and higher levels of organizational management take a priority. Masculine and feminine, therefore, do not become oppositional to one another, but rather complement each other in the decisions made and actions taken as a whole organization (Brown \& Light, 2012). Feminine leadership styles such as practicing care must take a center role in nurturing school staffs. A multidimensional awareness supports this approach. 
Kruger (2008) stated that "recognizing and accepting" the diversity that is coherent in the gender dichotomy of leadership will lead to a greater appreciation of both masculine and feminine styles (p. 166). Ayman and Korabik (2010) also highlighted the significance of diversity in leadership teams stating that this will also lead to harmony in organizations as a result of the natural symbiotic relationships that exist between male and female (Ayman \& Korabik, 2010). A harmonious leadership approach should and must lead to harmony amongst stakeholders within the organization. Ultimately, the investment in cohesion will result in increased buy-in to the common vision and mission. Kruger (2008) argued that diversity in leadership will also impact the school as an organization and its ability to adapt and respond to challenges. Much of the literature abundantly supports feminine approaches in leadership and often describes masculine leadership as toxic; however, the latter is a powerful tool that should be utilized in both the appropriate time and setting (Ayman \& Korabik, 2010; Brown \& Light, 2012) Ford, 2008; Moreno \& Yanez, 2007)

Organizations cannot simply put females or feminine leaders in top-level management and expect that a multidimensional approach has been satisfied. One style cannot be alienated in support of another as there are masculine approaches that are considered effective and necessary for schools to function successfully (Ayman \& Korabik, 2010). Ayman and Korabik (2010) claimed that successful supervisors adopt both a task-oriented and transformational leadership approach. The effectiveness of leadership in this instance is greatly influenced by the inspiring of subordinates to contribute more to the common goal with the pressures of personal accountability (Ayman \& Korabik, 2010, pp. 164-165). The masculine attributes of leadership that have been 
imprinted on society throughout the history of organizational management plays a vital role in the success of leaders and their followers in the journey towards success.

Coleman and Pounder (2002) called the mixing of masculine and feminine into decision making a model of multidimensional leadership. School leadership must realize that there are several central impending factors that determine the organization's success: structural, human political, symbolic, and educational leadership (Coleman \& Pounder, 2002, p. 124). Each of these areas of leadership pulls from one or both of feminine and masculine traits. This is a multidimensional approach, where not one quality is upheld over the other, but rather reinforces the claim that each has a valued role in organizational leadership.

Multidimensional leaders utilize the five distinct dimensions of structural, human, political, human, symbolic, and educational leadership (Cheng, 1994; Sergiovanni, 1984; Coleman \& Pounder, 2002). Each plays a specific role in the augmentation of leadership and ultimately the success of organizations. Schools benefit greatly from leaders who understand policies and procedures in the form of significant structural components of school success. Empowering personnel through professional development and transformational leadership takes into consideration the significance of human leadership.

Organizations are political bodies, where leaders must bargain and allocate resources in order to build teams and coalitions (Bolman \& Deal, 2008). Coalition building is an important aspect of political leadership, where the team of leaders within a school realize that the buy-in from stakeholders will be increased with an aware and adept group of individuals heading departments and the institution overall. School leadership must also be mindful of the culture of a school and know that their actions are being 
watched and often criticized. Symbolic leaders take the opportunity to influence members of the school through special rituals and activities. The final dimension of leadership involves informing subordinates through education. Educational leadership requires individuals to be effective communicators (Cheng, 1994; Coleman \& Pounder, 2002; Sergiovanni, 1984). The literature conveys the importance of both masculine and feminine approaches in these types of organizational leaders.

There are times when a power over (masculine) is the correct approach and others when a power through (feminine) is the best course of action (Coleman \& Pounder, 2002). To praise one approach, while devaluing the other, would be "missing the point" (Coleman \& Pounder, 2002, p. 128). The demand is for individuals who head schools and departments to not only recognize the strengths of the masculine and the feminine but also to deliver leadership with a harmonious respect of the two. The complexities of today's society that have placed great demands on public schools, now call for a new type of leadership. The literature suggests that these individuals must not utilize a single gendered style, but instead offer an approach that adopts what researchers have called "androgynous leadership" (Ayman \& Korabik, 2010, p. 165). Multidimensional leadership is evident in many positions within school districts.

The top-level managers typically are recognized as organizational leaders. They are responsible for supplying a vision and facilitating the processes to obtain set-goals. They are required to monitor the progress towards expectations and are ultimately responsible for the success of the company or institution. Despite the assumption that they are leaders, they may not in fact be able to lead. The false assumption that managers inevitably lead negates the multidimensional approach that is required to meet the needs of 
a multicultural complex world based on an ever-changing landscape (McNamara \& O’Hara, 2008). It takes more than titular leadership to accommodate the demands placed on public schools. Therefore, this research incorporated the many aspects of what Sergiovanni (2009) called a web of influence in school leadership to fully understand the picture surrounding current school leadership. 


\section{CHAPTER III}

\section{METHODOLOGY}

The purpose of this study was to uncover the approaches that school leaders use in decision making. The masculine and feminine leadership paradigm was explored to understand if leaders use multidimensional decision making. The literature recognizes that both masculine and feminine leadership approaches have benefits and restrictions. The research also consistently agrees that leadership plays a vital role in the productivity, effectiveness, and cultural morale of an organization. Leadership, therefore, must be an inalienable factor to the successful operation of a school. Because school leaders impact decisions at the highest level, this provides further reason for defining and building consensus around the decision-making processes of school leaders.

If society demands that schools improve, then the leadership approaches associated with successful schools must also improve. The evidence presented during the review of the literature has conveyed the connection between leadership approach and personnel response. A school is able to improve student achievement by understanding the approaches associated with masculine, feminine, and multidimensional decision making. Although some of the supporting literature highlights male and female leaders, the present study did not look to link causality between the sex of the individual with an expected gender-approach to leadership. Instead, it is accepted that sex may influence, but it does not ultimately determine the leadership approach of the individual. It is under the scope of responsibilities of the school leaders to decide how they will become a part of positive change in schools (Reed, 2012). 
The researcher looked directly at the masculine and feminine leadership paradigm and highlights consistencies and themes among modern-day leaders. The emphasis on not only school principals, but also the many individuals who contribute to decision making, conveys the scope of responsibilities placed on schools (Dahlvig, 2013). The expectations and complexities of operating schools have increased, partly or in whole, by the No Child Left Behind era and the stigmatism of an over-assessed and data driven public education landscape. The often overwhelming pressures placed on the local school house by federal, state, and local mandates that demand change in a time where the prescribed pathway is consistently contested. School leaders must not only understand their personal leadership style, but also be adept at determining the most appropriate leadership approach to the environments in which they lead.

The researcher looked to identify the styles of leadership that are meant to accommodate the needs of schools in a complex society (Coleman \& Pounder, 2002). These complexities are exemplified in the growing demands on the public education system and the paradox of developing fully rounded students by means of emphasizing testing and standardization (Barnett \& McCormick, 2004). School organizations must align personnel to combat the increasingly complicated task of adjusting for achieving measurable gains, while welcoming students from every demographic and background into the educational setting. Society not only requests, but requires the acceptance of all students and demands that schools adapt an approach that is proactive, flexible, and deliberate. The paradoxical environment asks schools to no longer simply respond to these demands, but to put forth plans to accommodate all learners, accelerate towards success, and to exercise a reflective practice to remediate any deficiencies. The only way for 
schools to take the appropriate steps to meet these difficulties is to accept their role as organizations and to empower key individuals to be leaders in schools (Sergiovanni, 2009).

\section{Research Questions}

The research questions posed in the present study were utilized to ascertain the characteristics of current school leaders. Specific emphasis was placed on masculine, feminine, or multidimensional approaches. The current study utilized the supporting literature to identify these characteristics. This research looked to highlight consistencies in the types of approaches used by participants, by looking at how participants responded and repeated certain leadership decision making traits in their answers. The following questions determined the entire scope of this research:

1) On the basis of the literature, what approaches of current Miami-Dade County Public School leaders may be understood to be masculine, feminine, or multidimensional?

2) On the basis of the literature concerning masculine, feminine, and multidimensional leadership approaches, given information about other leaders' responses, how do Miami-Dade County Public School leaders report and explain their leadership decisions? 
The researcher sought to ask more questions and uncover more relevant factors during the investigation process. The methodology chosen for this research lent itself to theory building and sought to contribute to the body of literature relevant to public school leadership.

\section{Method and Process}

The current study utilized a modified electronic Delphi technique to analyze the ideas and themes relevant to school leadership decision making. Firstly, an attempt to identify whether school leaders utilize a masculine, feminine, or multidimensional approach was explored. Secondly, the researcher asked participants to report and explain their decision-making process. By applying the aspects identified by the supporting literature, these data unveiled the type of leadership implemented by each individual.

\section{The Delphi Technique}

This study utilized the Delhi technique to uncover and build a consensus around the themes of masculine, feminine, and, multidimensional leadership. Because leading involves many contradicting details as outlined in the paradoxical nature of leaders (Bolman \& Deal, 2008), this study offers a description for how current leaders operate in Miami-Dade County Public Schools. The use of a Delphi technique allowed for participants to answer, define, clarify and revisit their approaches to the matter being explored, which was congruent with the supporting literature that utilized the same method (Taleb, 2010). 
The follow explanation provides information surrounding what comprises the steps in a Delphi technique. The method utilized builds consensus by first polling participants by use of a questionnaire, which sets the tone with a set of open-ended questions. Building consensus and theory building guides the research by unveiling certain themes that are found to be relevant during the course of the research (Hsu \& Sandford, 2007). The participants are given feedback on the questionnaire that provides them with information concerning commonalities and similarities in responses among the candidates. The information about any unique or outlying perspectives can also be presented; however, this is only done if the researcher feels that this will fuel the progression of the study. The Delphi technique then asks the participants in following round to answer questions by using a survey or questionnaire about aspects that were highlighted in the initial round. During each cycle, or round as it is referred to, the researcher will approach the participants with a more formulated and defined viewpoint of previous questions, items and themes; therefore, the evidence collected is used to develop consensus and/or build understanding around a particular topic of interest.

\section{Why Modified Electronic Delphi?}

Criticisms of the Delphi technique include that individuals with overpowering personalities and those who appear to be recognized as the foremost experts in the field tend to dominate group discussions and the data collection process. The many perspectives of the group are drowned out by domineering personalities, which may lead to the persuasion of participants involved in the study (Christman \& McClellan, 2012). Even though this is a legitimate criticism, a modified electronic Delphi takes the group setting 
and transfers it to a digital one. The researcher, therefore, becomes a facilitator of the process and mediates the conversation between members. The researcher also monitors that time is not lost as a consequence of off-task activities and sidebar conversations, which could cause distraction and a lack of focus during participant interactions. The electronic or digital style of this study allows for specific deadlines to be set and it accommodates the busy life-styles of today's educational leaders.

Specific criticism of this type of study emphasizes the length of time and amount of constant input from participants that is required; however, a modified electronic approach allows for an alternative approach that remedies some of these challenges (Christman \& McClellan, 2012). With specific calendar date deadlines and timelines set for each round of the modified Delphi technique, participants were given one to two weeks to complete each segment. Additionally, the digital approach also simplified the communication process, as notifications were sent through email. Data were collected by use of the format provided on Google Forms for each round. This online structure allowed for synchronicity to the data collection process.

\section{Procedures}

This study implemented a four round modified electronic Delphi technique. The Delphi technique typically calls for a minimum of three stages, where an optional fourth is added for clarification and it also gives the participants an opportunity to reflect and expand their thoughts (Hsu \& Sandford, 2007). The present study utilized an optional fourth round, based on the idea that self-reflection leads to more appropriate leadership 
(Maslow, 1991). Each round offered a unique opportunity to explore the participants' ideas behind their personal decision making.

\section{Round One}

The first round answered both research questions and lasted for a duration of one week. This round consisted of a 10 part open-ended questionnaire administered via the Google Forms online format. Each item correlated with one or more leadership approaches that have been identified as masculine, feminine, or multidimensional by the supporting literature. The questions were tailored specifically with an objective of answering the two previously identified research questions. The initial questionnaire is available in the Appendix W. Examples for the preliminary questionnaire included:

1) During the course of the school year, is it more important to be adaptable or stay loyal to the vision in which your stakeholders are committed?

2) Is succeeding the ultimate goal in endeavors pursued at your school and how do your decisions reflect obtaining set objectives?

3) When seeking to foster a positive environment, explain how important it is to seek out the input from every individual in the building in maintaining a desired overall school culture:

Question one from above includes both the masculine approach of staying loyal to organizational vision, as well as the feminine of adaptability and therefore the question can be considered to be presented in a multidimensional way. As for the second example question, the reader can observe only the masculine approach in perseverance and dedication to set objectives. This question is recognized as having a masculine tone. The 
final example from the questionnaire conveys only the feminine approach of building a positive environment with an emphasis on valuing every individual's input and therefore represents a feminine-styled question. The rest of the questionnaire is available in Appendix W. The corresponding letter representing each leadership approach has been added in parenthesis behind each question. This feature was only added following the study and is present only for the readers of this body of work. The details surrounding these letter abbreviations are explained in the Findings chapter.

The answers provided were coded not only for commonalities, but also in accordance to themes related to masculine, feminine, and multidimensional leadership that have been identified by the supporting literature. The explanations provided by the participants were utilized, along with the specific leadership approach identified after the researcher analyzed the questionnaire item responses, to create a multiple-choice survey for round two. Participant responses were coded into one of the major reporting categories of masculine, feminine, or multidimensional leadership approaches. The guiding details behind this analysis are available in the findings chapter. The most common leadership approaches found in item responses were used to help guide and determine the progression of the research. Table 1 conveys the leadership approaches and their perceived identification based on the supporting literature. 


\section{Table 1 Leadership Approaches/Traits and Reporting Categories}

\begin{tabular}{|c|c|c|c|}
\hline Approach/ Trait & Masculine & Feminine & Multidimensional \\
\hline Voice for Minority & & $\checkmark$ & \\
\hline $\begin{array}{l}\text { Appreciating Relationships/Valuing } \\
\text { People }\end{array}$ & & $\checkmark$ & \\
\hline Adaptable/Flexible & & $\checkmark$ & \\
\hline Collaboration & & $\checkmark$ & \\
\hline Believing in Others & & $\checkmark$ & \\
\hline Support of Family & & $\checkmark$ & \\
\hline Role Model for Others/ Self-Image & & $\checkmark$ & \\
\hline Self-Motivated & $\checkmark$ & & \\
\hline Commitment to Vision & $\checkmark$ & & \\
\hline $\begin{array}{l}\text { Sense of having to succeed/Failure } \\
\text { not an Option }\end{array}$ & $\checkmark$ & & \\
\hline Positive Self-Concept/Self-Efficacy & $\checkmark$ & & \\
\hline Persistent & $\checkmark$ & & \\
\hline Perseverance & $\checkmark$ & & \\
\hline Efficiency and Effectiveness & $\checkmark$ & & \\
\hline Reflection & & & $\checkmark$ \\
\hline Experiential Maturity & & & $\checkmark$ \\
\hline Planning/Prioritizing & & & $\checkmark$ \\
\hline Keeping Perspective & & & $\checkmark$ \\
\hline Optimism/ Positive Mindset & & & $\checkmark$ \\
\hline
\end{tabular}

\section{Round Two}

The second round survey, available in Appendix X, presented a specific scenario related to the leadership approaches from round one's questionnaire items. 
The survey items asked the participants to make a choice based-off of four answer choices. Each answer choice coincided with masculine, feminine, or multidimensional leadership with the last option offering the opportunity to participants to write their own decision. This answer choice offered space to write the best possible decision in relation to the scenario presented. The second round survey was administered through Google Forms and encompassed 10 items that were correlated with the previous round's questionnaire. The second round provided further data from the participants to answer the research questions. For example is the first question in the Situational Decision Making Survey:

1. A directive has been sent straight from the school board to be implemented within the school district, you:

a) independently study the request and come up with a game plan to present to your school/department. (Masculine)

b) quickly gather a leadership team to disseminate responsibility.

c) devise a plan and further develop the appropriate steps with colleagues to implement an achievable plan.

d) Other. Please explain an alternative decision.

The situation presented above correlates with round one's questionnaire by focusing on the participant's reliance on a team/collaborative approach (feminine style), independent decision making (masculine style), or incorporating both independent and collaborative strategies to decide the most appropriate leadership approach (multidimensional style). Each of these possible leadership approaches are related to an answer choice: a) masculine in choice b), feminine in choice c) multidimensional and choice d) allowing for the participant to expand on a different decision.

Round two lasted a duration of one week. The results to the survey presented the thoughts expressed by each participant and the questions were framed in a way that asked each individual to further reflect on the most appropriate decision-making answer. Following this second round, the data received was analyzed by majority responses. 
Answers conveyed were categorized by use of the supporting literature as masculine, feminine, or multidimensional. The fourth 'other' option asked participants to expand on a more appropriate decision. The responses were coded to determine whether the participant emphasizes one of the reporting categories over the others. Again, the majority responses for each item were used to help guide and determine the progression of this study.

\section{Round Three}

A third round lasted one and a half weeks and presented the mean and mode of the survey responses to the participants by use of Google Forms. Participants were presented the most common responses by percentage via a pie chart for each survey question item. Round three required each individual to look at the response percentages for each item and asked to reflect on the results. This served as a digital interaction during the course of the Delphi technique. Their reflection on each survey item was collected via open-ended form that asked participants to reflect, expand, or further define the attributes of the decisionmaking process of each scenario. Each individual was given the opportunity to revise or reaffirm his/her response based on the overall responses. The data collected was coded based on the previously identified reporting categories. A final round was utilized to convey the progression of the study to the participants and receive their final input on majority responses. The presentation provided for participants for round three is available in Appendix Y. 


\section{Round Four}

The fourth and final round lasted a duration of a week and a half. It presented certain specific responses for each item that were selected by the researcher.

During a Delphi technique both common and uncommon statements may be shared to help progress the study. The researcher utilized the most common leadership approaches of the responses shared. As previously mentioned, outlying opinions are important and can be a vital part of developing consensus in a Delphi technique study (Christman \& McClellan, 2012). The utilization of this information allowed for more relevant information to be offered with another opportunity for participant reflection. During the final round, participants were invited to make observations for round three's results; however, a response was not necessary to complete the study (Hsu \& Sandford, 2007). The information presented to participants for this round is available in Appendix Z. Each round implemented during the course of this study allowed for the data gathered to answer the two research questions. Each round was initially scheduled to last only one week's time; however, this was extended by half a week for both rounds two and three to receive full participation in the study.

\section{Participants}

This study solicited the participation of total of 20 participants fulfilling the many different positions that compromise school leadership. Individuals fulfilling the following roles participated in the present study: one administrative director, three principals, eight assistant principals, two instructional coaches, two district support personnel, one community involvement specialist, one team-leader, and one counselor. Because each of 
these individuals are responsible for implementing initiatives, organizing personnel, and applying resources to achieve goals, an emphasis on specific roles of participants was not necessary to answer the research questions of this particular research. Participants were assigned to location from across Miami-Dade County Public Schools and not at one particular school location.

The participants were informed that the individuals participating in the study were school leaders ranging from curriculum support to school administrators. Subject participation was garnered through email invitation and face-to-face invitation. The researcher communicated with the participants by email, phone conversation, text message, or Google Form notifications to facilitate full participation in the study. The individuals who volunteered for the study were notified of the research title dealing with masculine, feminine, and multidimensional leadership; however, at no time were the individuals notified concerning how their responses were coded. This decision was made to avoid participant bias or participants falsely identifying with one leadership approach over the other based on preconceived notions or stereotypes.

\section{Limitations}

It is important to note that even though the data for the current study will be collected and analyzed by the use of both quantitative and qualitative research methods, the sample size may prevent the results from robust and generalizable beyond the current setting. The researcher has attempted to uncover concepts and build theory around sexrole generalizations and multidimensional leadership. Further researcher with increased 
sample sizes utilizing the findings to the present study will strengthen the ideas and concepts discussed in Chapter V. 


\section{CHAPTER IV}

\section{FINDINGS}

Data were collected over the course of a 6-week period with all 20 participants completing the three required rounds of the Delphi technique. Twelve of these individuals chose to complete round four, which was a voluntary round. Upon an informal effort by email and/or phone conversation, the remaining eight participants who did not attempt round four claimed that after a review of the information they had "nothing further to add" and "the results mirrored my overall impressions."

The research conducted during each round provided a unique snapshot into the decision-making processes of the participants. With each subsequent round, participants were allowed the opportunity to refine their impressions in regards to questions and survey items. Participants were able to expand on their thought processes and reflect on the majority results received from all individuals. This chapter will firstly describe how the results will be reported in accordance to the two research questions, secondly define specific terms and themes the terminology that was used to analyze the data, and lastly convey the results of each round. By providing the results in the final sequence of this chapter, the intent is to provide a smooth transition for the following chapter, which includes the discussion of the findings and significance of the study.

\section{Introduction to Results}

There were two guiding research questions that determined the scope of the research as well as the purpose of each round. The first research question: "Based on the literature, what approaches of current Miami-Dade County Public School leaders may be 
understood to be masculine, feminine, or multidimensional?" was answered over the course of the entire study. The first round was implemented to offer insight to help answer the first research question by creating a leadership decision-making profile for each individual that identified each participant as masculine, feminine, or multidimensional; however, throughout the course of the study, the data conveyed an evolution of the way participants reported and explained their thinking. With each subsequent round, participants offered further insight into their leadership decision-making processes. For the second question, "Based on the literature concerning masculine, feminine, and multidimensional leadership and information about other leaders' responses, how do Miami-Dade County Public School leaders report and explain their leadership decisions?" this was answered through personal descriptions in round one, decision making choices in round two, and the reflection and refining of decision-making approaches in rounds three and four.

The first round, The Leadership Decision Making Questionnaire, provided an open-ended format to participants with 10 questions that were developed based on several approaches of school leaders who were previously identified by the supporting literature as masculine, feminine, or multidimensional. The open-ended style of the first cycle to the study offered an opportunity for participants to react to the questions by reporting their thought process surrounding these previously identified leadership approaches. This allowed the researcher to develop a preliminary outlook for how each individual participant approached decisions in regards to the three reporting categories of masculine, feminine, and multidimensional. The answers received via Google Forms results, along with the supporting literature, were used to create the second round's Situational Decision 
Making Survey. This process involved utilizing the leadership approaches brought forth in the first round questionnaire and correlating them with a specific scenario. Each of the answer options related to either a masculine, feminine, or multidimensional leadership decision-making approach.

The survey presented 10 specific scenarios aligned to the approaches from the 10 questions presented in round one. This allowed a smooth transition between rounds and also contributed to the ability of the researcher to develop the subsequent rounds of two, three, and four in the study. Each item within the survey was presented with an answer choice that corresponded with a masculine, feminine, multidimensional leadership. There was also an option labeled as "other." This option asked participants to expand or further define the decision-making approach that they believed more suited the situation that was presented. Even though only a few participants chose the 'other' option, this resulted in a stressing masculine, feminine, or multidimensional leadership, rather than an expanding upon one of the subcategories that will be detailed later.

The second round results provided through the Google Forms format offered a snapshot of the decision-making approaches of the participants with an emphasis on the specific scenarios presented. This contrasted with the first round, which asked participants to describe their thought process surrounding certain approaches and themes in leadership. This resulted in data that was quantifiable in regards to the prescribed reporting categories, which ultimately led to a comparison in decision-making approaches between the first two rounds. A third round was created by presenting the majority responses of each survey item. 
The third round Survey Reflections, once again allowed the participants to respond to the data that had been progressively synthesized through the previous rounds. Participants responded in open-ended form via the administration of a Google Forms format, allowing them to reflect on most common leadership approach prevalent in responses and either concur or further refine a leadership approach that best matched their decision-making process. This was similar to round one in that it asked each individual to expand on his/her thought process in making decisions. Some participants took this opportunity to agree with the majority opinion, which was an opportunity to emphasize the masculine, feminine, and/or multidimensional approach of their decision making. Round three responses were analyzed similarly to rounds one and two by coding each response with one of the identified reporting categories that will be described below. The third round was the last required portion of the study. A fourth round was implemented to solidify the findings being developed and to collect more information and provide support for further discussion.

The fourth round, Final Reflections, utilized three to four specific answers provided by participants and presented them with the majority responses of round two for each of the 10 items of the survey. This round was also administered and data collected via Google Forms. Specific excerpts received from round three's responses were selected by the researcher based on how clearly they reflected a particular leadership style and presented them for final reflections. For the 12 of 20 participants that chose to participate in this summative round, the data presented a final opportunity to take a snapshot of the overall decision-making processes of current school leaders. 


\section{Reporting Categories}

The three original reporting categories of masculine, feminine, and multidimensional were expanded to include five more reporting subcategories, which better represented the decision-making process of the participants. These will be identified below. The analysis of participant responses utilized the traits identified in Table 1. The researcher used this information to code responses throughout the study and determine whether the answers provided by participants fell into any of the categories that are described in this section below. Their entire answers to questions were coded, in which responses often were identified to contain multiple leadership approaches. The following will describe how the leadership approach categories were both identified and expanded to better understand and analyze what the participant responses were unveiling.

The masculine approach was seen in two ways: masculine (M) and masculine/multidimensional (M/U). Masculine/multidimensional was conveyed through responses that were masculine, but had undertones of multidimensional attributes by the use of specific terms that were previously identified in the supporting literature. Congruently the feminine approach was also seen in two ways: feminine (F) and feminine/multidimensional (F/U). Even though the description of multidimensional leadership is defined as the infusion of both masculine and feminine approaches into decision making (Coleman \& Pounder, 2002), both M/U and F/U differ in that each overtly uses one side of the masculine/feminine leadership paradigm to describe his/her leadership decisions, while referring to some specific traits of multidimensional leaders. It is important to note that the supporting literature does recognize certain traits as multidimensional without including specific masculine or feminine approaches. To better 
clarify the justification for the expansion of the leadership approach reporting categories, the reader is encouraged to refer to Table 1 and the various approaches attributed to masculine, feminine, or multidimensional. It is also vital that the reader apply the details of Table 1 to understanding the following details each reporting category and subreporting category.

An example of a response that was found to represent a F/U leadership decisionmaking approach is as follows:

"Going home every night knowing that I helped someone get better is the daily goal. What is success though? Perfect EOCs scores for all students or Making sure all students graduate with a plan that will enable them to become a lifelong learner and a productive citizen."

This participant has rejected the masculine aspects of question 7 of the questionnaire and highlighted his/her believing in helping others. This feminine approach is followed by a response that details keeping perspective and looking at situations holistically, which is a multidimensional characteristic. The following is an example of a participant response that was coded as $\mathrm{M} / \mathrm{U}$ :

"Ultimate goals and endeavors at my school must include the ultimate vision to succeed because in essence success is student achievement which is the ultimate goal and the obligation we all have to each and every child that comes through our doors. "

This response conveys an emphasis on the masculine approach of maintaining loyalty to the vision to explain his/her decision-making process. The participant incorporates experiential maturity, which is a multidimensional approach into the response. This 
participant ultimately emphasizes the masculine approach in decision making but has multidimensional undertones. Underlining multidimensional details such as this swayed the researcher's coding of responses. Because of this, the researcher chose to recognize subcategories that existed in the data.

The leadership approach of multidimensional was further divided and conveyed in four distinct ways: multidimensional (U), which represents answers that included specific traits identified by the supporting literature as multidimensional, but lacked specific reference to either the masculine or feminine approaches, multidimensional/masculine (U/M) conveyed with approaches of multidimensional leaders with an emphasis on the masculine, multidimensional/feminine (U/F), which was found in answers that were multidimensional with an overall assertion to feminine approaches, and multidimensional/masculine/feminine (U/M/F) from participants that described leadership decisions by the use of all three categories. The following response was coded as $\mathrm{U}$ :

"Life happens and leaders must be able to adjust. It is important to have a balance that is based on needs and situations. It is never cut and dry, all situations are different."

This response conveys the multidimensional approach of keeping perspective. The participant does not commit to either a masculine or a feminine approach in his/her thinking, yet there is an obvious cognition of relevant leadership approaches that do not coincide with one type of leadership over another. The following conveys a participant's response that was coded as $\mathrm{U} / \mathrm{M}$ during the data analysis phase of this study:

"Once the vision is approved upon by the stakeholders is important to stay loyal to the vision while making notes of what is happening in the process of shaping the 
vision. The notes during the process can be revisited at the end of the school year to discuss what worked and what adjustments need to be made."

The participant has emphasized staying loyal to the vision of the organization, which is a masculine approach. The individual follows up his/her primary assertions with the multidimensional approach of making adjustments based on need. This correlates with the multidimensional trait of experiential maturity observable on Table 1.The following is an example from one participant's response of an answer that was coded as U/F:

"Flexibility is more important to a large degree, wherein remaining loyal to a particular vision may not be conducive to achieving the desired outcome. Adaptability ensures that someone can rise to the occasion when things do not go according to plan. Remaining loyal to a vision can also lead to tunnel vision, whereas flexibility allows people to "think outside the box" and consider alternatives at all times.”

The participant has chosen to emphasize the feminine approach of flexibility, but goes on to show that he/she is utilizing the multidimensional trait of keeping perspective when the masculine approach of loyalty is downplayed. The individual has considered alternative decision-making approaches and has used the multidimensional traits of experiential maturity and keeping perspective as driving factors to decision making. The following is an example from the study participants of a response coded as $\mathrm{U} / \mathrm{M} / \mathrm{F}$ :

"One must be careful to not vary too greatly from the original vision. Flexibility must be observed as school, student, staff, and stakeholder needs change throughout the year in order to address and meet those needs. It is important to 
remind stakeholders of the vision and the final goal, but apply flexibility when working towards that goal."

This response first conveys the masculine approach of loyalty to vision, but follows this assertion with the emphasis on the feminine approach of flexibility. By describing the importance of recognizing the balance of the two through experiential maturity, response is Multidimensional by including both masculine and feminine approaches in his/her response.

It became apparent to the research that keeping with the original three reporting categories, or leadership approaches in the analysis of the participants' responses would not be advantageous to the understanding of how these particular school leaders made their decisions. Recognition of the subcategories outlined above served the progression of this study and furthered a more in-depth analysis of the data collected. It also provided a more accurate snapshot of decision making of current school leaders.

\section{Presentation of Findings}

The results will be presented for this study in several distinct sections. First, an introduction for each round will provide details surrounding each round. Second, the findings will be conveyed with an overall picture of participant leadership style approaches. Following this overview, the third portion of each round will present the results of each reporting category of masculine, feminine, and multidimensional. This will include the status of participant choices in each of the previously identified reporting subcategories of $\mathrm{M} / \mathrm{U}, \mathrm{U} / \mathrm{F}$, etc. A review of the overall results in relationship to the

research questions will be discussed in the following discussion chapter. It is essential to mention that the approaches highlighted in Table 1 were used to code each answer 
provided by participants. Readers should refer to this table for the following presentation of the findings.

\section{Round One}

The Leadership Decision Making Questionnaire focused on a list of pre-identified attributes that represented different gendered approaches to leadership. The evidence presented in Table 1 recognizes particular approaches as being masculine, feminine, or multidimensional. One or more of these leadership characteristics were utilized for the creation of each item on the first round questionnaire. Available in Appendix W, six of the 10 questions were worded in a way that reflected both a masculine approach and a feminine approach in the question details Appendix $\mathrm{W}$ also has the corresponding letter abbreviation for each category in parentheses following each question. These details were only added for the benefit of the understanding of the readers of this research. These were questions $2,3,4,6,9$, and 10 . Two questions numbers 1 and 8 were worded in with an emphasis on the feminine with on worded in the masculine. Responses measured the approaches that were emphasized on a masculine-multidimensional-feminine spectrum.

In regards to the three main leadership approach reporting categories, round one resulted with a higher number of participants expressing their decisions with feminine leadership approaches. Twenty-nine percent of the overall responses to the questions for the first round were coded as reflecting a feminine leadership approach (F). For the two questions that were worded reflective of a feminine leadership approach the respondents overwhelming used these traits to describe how they made decisions. Responses that reflected a feminine leadership approach were the majority on three of the 10 questions with three additional questions equally reporting a feminine approach. Individuals 
emphasized the involvement of all stakeholders in decisions, which highlighted both valuing and believing in others. Participants shared themes of "involving members of the team" and recognizing the importance of being a model of expectations. The following participant emphasized the benefits of sharing responsibilities through the delegation of tasks:

"Involving others and delegating tasks ensures that teachers are not burned out with responsibilities and are overwhelmed with too many tasks. Sharing tasks gives the ones who are consistently working a much needed break and it fosters new ideas and creativity versus having the same people doing the same thing." When asked about the importance of valuing family in relation to completing tasks at work in question 6, the responses included "family is the number one priority" and "stakeholders are encouraged to value family over work." The second highest reporting category was multidimensional leadership approaches (U), which resulted in $26 \%$ of total responses

There were six questions that were presented with either the masculine and feminine approach included in the question details or a multidimensional trait being the focus. Therefore, the questionnaire was written with a multidimensional perspective. Despite this, only three questions had a majority response of multidimensional leadership approaches. In these circumstances, participants used the keeping perspective approach to explain their thought processes. Their responses included "identifying key individuals to complete tasks" rather than including all employees. Respondents also emphasized "knowing your stakeholders," which conveyed the approach of experiential maturity. Participants also mentioned the importance of being reflective to shape the most 
appropriate approach for particular situations. The lowest of the reporting categories for round one was masculine (M) with a $20 \%$ of the responses.

Two questions resulted in a majority of masculine responses. With these questions, participants noted that "all endeavors must include the ultimate goal" and "achievement of set objectives and goals" are the main points of emphasis that influence decision making. This participant response reflects the masculine approaches of accountability and followthrough:

"Delegating without monitoring is a recipe for disaster. In the daily course of a school day, many operational and curriculum issues arise; if administration delegates tasks and do not monitor the completion or the progress, the end result is never accomplished as staff will quickly realize that no one is monitoring the results. It could be as simple as asking a custodian to clean a bathroom that was found dirty the night before. The Assistant Principal is asked to deal with the situation and he/she emails the custodian but never goes to inspect the bathroom. The bathroom will more than likely not be cleaned."

The responses coded under the masculine reporting category reflected perseverance and commitment to the vision as guiding principles in leadership. In question 9, that asked whether it is more important to be respected or loved, the participants emphasized roles and responsibilities in expressing an answer.

"As a leader earning the respect of the stakeholders is imperative to being able to accomplish the goals of the organization. Respect will motivate a stakeholder to act, whereas, love may not." 
"It is more important to be respected. When trying to lead others, they must feel respect towards the leader. The leader must be seen as fair, knowledgeable and caring."

"I think it is more important to be respected because a certain level of love can come from the respect people have for you as a leader."

This masculine approach was represented by fulfilling the role as a leader within the education environment and that respect "comes with the territory." Through further analysis of the three major reporting categories, it became more apparent that subcategories existed. The inclusion of these subcategories would more accurately present the leadership styles of the participants.

Within the multidimensional approach $13 \%$ of responses utilized both masculine and feminine details in their descriptions (U/M/F). This was evident by answers that for example mentioned not only receiving buy-in from all stakeholders, but also emphasizing the need for there to be a top-level decision maker or makers to put items into action was a significant part of their decision-making process. This stressed both the masculine and feminine, while also illustrating the multidimensional approach by keeping perspective. $6 \%$ of participant responses were coded as multidimensional with an emphasis on masculine decision making (U/M). These answer choices reflected keeping perspective and experiential maturity with significance given to "ensuring commitment to goals" and weight being given to "efficiency and effectiveness."

The last reporting subcategory of multidimensional leadership included keeping perspective, optimism, maturity, and also emphasizing the feminine approach (U/F). Four percent of responses reflected a mixture of multidimensional decision making with the 
importance of being flexible in situations and "being willing to change." Both the masculine and feminine categories were further divided into one subcategory each of $\mathrm{M} / \mathrm{U}$ and $\mathrm{F} / \mathrm{U}$, where the main category was dominant while reflecting some multidimensional leadership attributes.

Those who expressed masculine approaches with an undertone of multidimensional $(\mathrm{M} / \mathrm{U})$ totaled $3 \%$ of the total responses. This was evident with a focus on "staying loyal to the vision" in each endeavor, while "sometimes acknowledging" that "flexibility and transparency are important." The flexibility emphasized shows the participant keeping perspective. At the opposite end of the spectrum, those who exhibited feminine approaches with underlying multidimensional comments added to their responses (U/F) totaled $1 \%$ of the responses. In a similar example, these individuals emphasized seeking the "input of all stakeholders" in achieving tasks, while learning to keep perspective. Involving each stakeholder highlights the leader's belief and value in others. Data for the 10 questions for the first round are illustrated in Figure 1. 


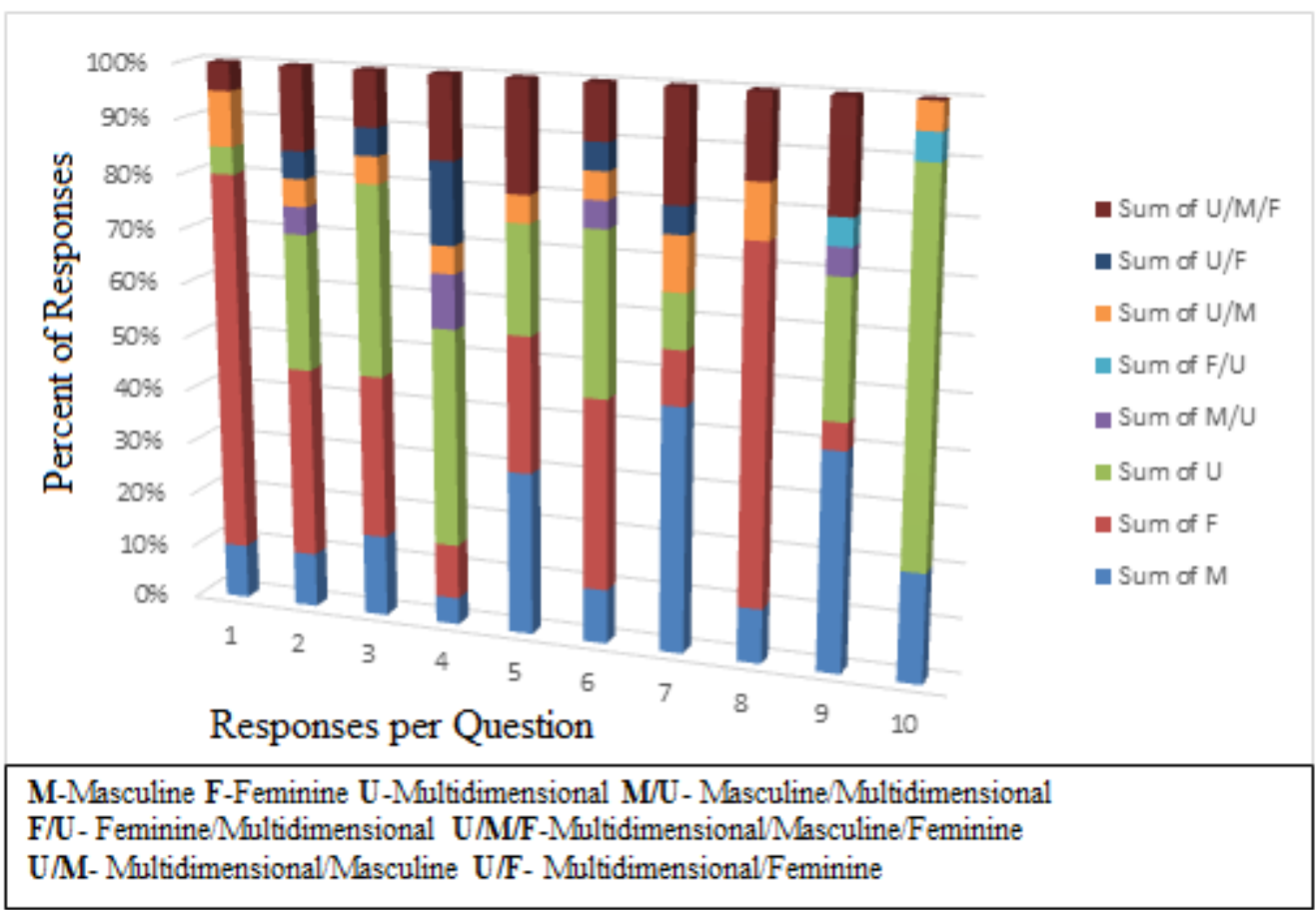

Figure 1 Round One Overall

\section{Round Two}

The Situational Decision Making Survey required the participants to respond to a set of situations with a leadership decision that appropriately matched their most likely approach. Each situation item corresponded directly with the leadership approaches represented in the question items from round one. The questionnaire in the first round allowed participants to express their decision making, whereas the results for round two's survey required the participants to choose an answer that can be coded as masculine, feminine, multidimensional. The "other" option includes was selection asked participants to explain an alternative decision to what was already presented.

The Situational Decision Making Survey concluded without any representation of the previously outlined reporting subcategories. Fifty percent of the total responses expressed the multidimensional options for participants. In these instances, participants 
chose the answer that included a masculine and feminine approach, rather than the choices that solely represented either one or the other. Six of the 10 survey items returned with the multidimensional answer choice as the majority consensus. In these circumstances, participants chose to "create a plan and further develop the appropriate steps with colleagues," individually write an announcement, and then "share with a trusted group of individuals to receive their input." In each of these cases, the participant emphasized a positive self-concept and belief-in-self before activating the use of a leadership team. For the second round, there was a drop in feminine leadership approach responses from $29 \%$ to $19 \%$.

No questions resulted in a feminine response as the majority for the second round; however one question resulted in $40 \%$ of participant responses finding it useful to "work with a group of individuals in a trusted group of colleagues" instead of emphasizing individual responsibility, which is considered a feminine approach. Although not the majority, this response conveyed the participants' reliance on valuing and believing in others. In contrast to the drop in responses reflecting feminine approaches, masculine approaches saw an increase in representation for round two.

The survey resulted in three questions having masculine leadership approaches as the most common in the answer responses. Additionally, $32 \%$ of the responses were found to be masculine. This was an uptick from $20 \%$ of the responses in the first round. In these questions, participants highlighted the importance of relying on their own capacity to make decisions. These answer choices also emphasized a positive self-concept when implementing an action plan. For round two, there were no results in regards to the subcategories. The answer choices allowed for participants to choose one of four choices, 
with one representing each of the major reporting categories and one last 'other' option that offered the opportunity to provide an independent answer. The last option was an opportunity to produce data for the subcategories; however, those who selected this choice expressed their situational decision making in terms of the major reporting categories.

Data for the second round is illustrated in Figure 2.

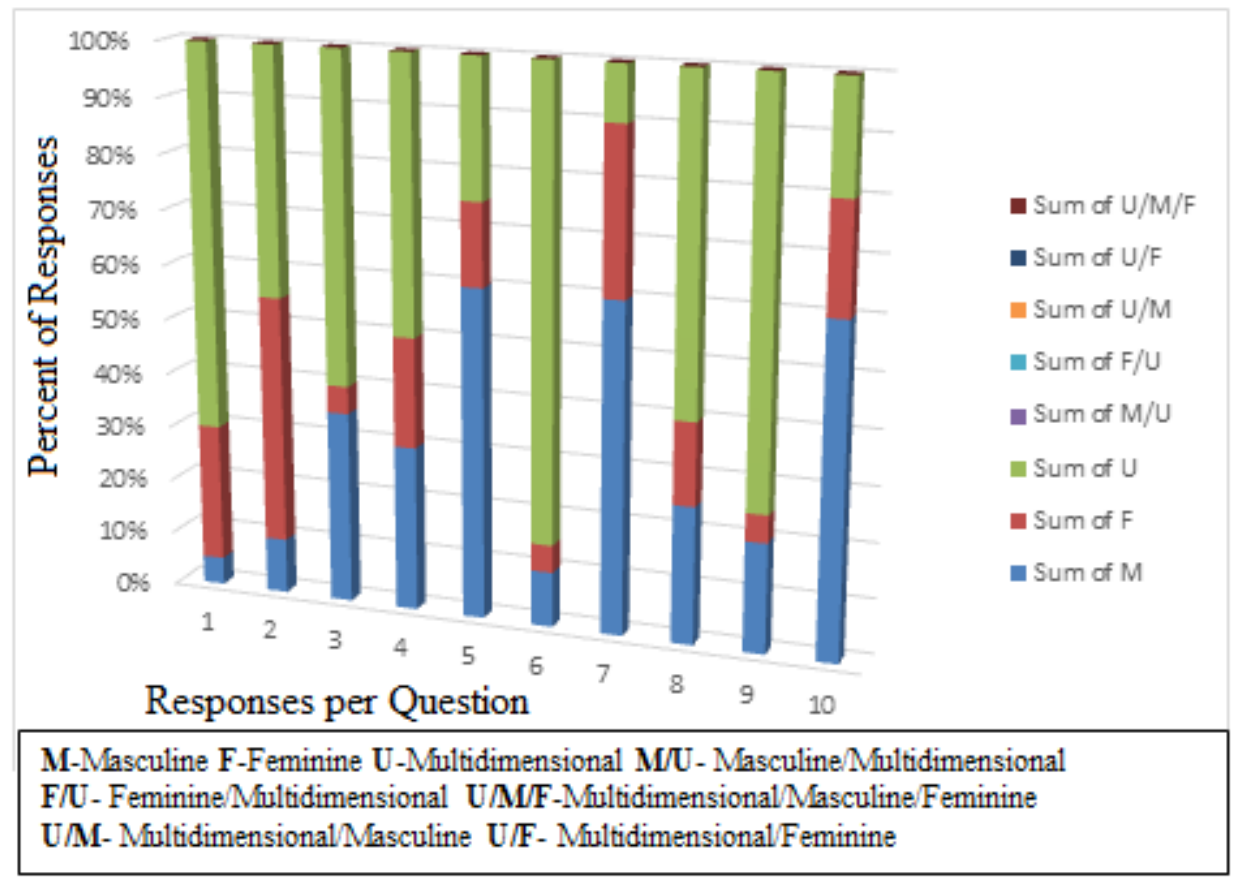

Figure 2 Round Two Overall

\section{Round Three}

The Survey Reflections utilized an image of each survey question from round two with a pie chart and description conveying the results from the previous round.

What was presented for round three is available in Appendix Y. Participants were able to see the percentage for each question and the answer choices chosen. This allowed them to reflect, revise, and/or re-emphasize what leadership approach was the most appropriate in their view.. Additionally, round three offered an opportunity to record a growing 
leadership style profile for each of the participants. Round three was the last required round. Because 12 participants participated in the fourth voluntary round, round three resulted in a final picture of the leadership decision-making processes of eight school leaders.

The reflections to the survey utilized an open-ended format for participants to report and explain their decision making. This resulted in multidimensional answers maintaining a majority with $36 \%$ of total answers. This was $10 \%$ more than the openended responses received in for round one's Leadership Decision Making Questionnaire. Six total questions returned with a majority of multidimensional responses: questions 2, 3, $6,7,8$, and 10 . The multidimensional responses received during round three emphasized the approaches of keeping perspective, utilizing experiential maturity, and being reflective. During this round, participants began to use new multidimensional language to explain decisions. For example, the concept of prioritizing became a prevalent theme across multiple participant responses. This was seen with "planning for effective results" and "prioritizing and delegating tasks whenever possible."

"As an administrator prioritizing and delegating tasks is imperative to the efficient completion of work functions. By managing time and efforts in a strategic manner, administrators are better able to complete tasks with the utmost efficiency." "Prioritizing your work then delegating is always an effective way to ensure work is not overlooked and deadlines are met. The duties of an administrator can be overwhelming and can become very hectic. Prioritizing and delegating is an effective strategy that provides others who are looking into becoming and administrator with experience." 
These participants have emphasized their understanding of leadership decision making by emphasizing priorities and the distribution of responsibilities. Prioritizing is an example of experiential maturity and keeping perspective and ultimately a multidimensional approach to leadership. Participants began to use words like reflection and the importance of "reflecting on the process" in this round, where it was not emphatically used in previous rounds. The third round saw an upswing in feminine responses when compared to round two.

Responses returned with $23 \%$ in the feminine reporting category, which was a $4 \%$ increase from the previous round. Three of the total questions resulted in participants emphasizing feminine approaches to decision making. In each of these questions, participants overall switched from round two's multidimensional approach to reiterating the feminine approach. This was evident with responses describing "collaboration with other professionals," "devising plans with colleagues," and asking stakeholders to play a major role in the planning and implementing process of decision making.

"I continue to state that it is crucial to have the participation of all stakeholders.

Therefore it is key to share potential decisions with others to seek their input in order to ensure success"

This individual has conveyed the importance of the feminine approaches of believing in others and valuing the input of employees. Masculine approaches overall fell in round three.

Round two's Situational Decision Making Survey result of 32\% masculine responses decreased to $20 \%$ of total answers for round three. Only one question returned with a majority of participants reporting the best course of action in terms of the masculine 
category. This particular question asked about the importance of believing in others in contrast to relying on one's belief in him/her. The scenario presented in the survey had resulted in $60 \%$ of respondents choosing the importance of relying on one's capacity to individually devise a plan to deal with an impending setback. This participant has described the appropriate response in masculine terms of reliance on one's self to derive confidence in decision making.

"What else is there to do? You wouldn't have been placed in that position if you weren't able to get the job done. Find a way and get it done."

Round three saw a similar result with $65 \%$ participants emphasizing this point. Responses emphasized "knowing your strengths" and management of duties as an important aspects of a leader's responsibility. This highlights the masculine approach of positive selfconcept and self-efficacy. The open-ended responses from round three returned with results that reflected the reporting subcategories.

Multidimensional leadership saw $10 \%$ of responses signifying both the masculine and feminine in their decision-making thinking (U/M/F). In two particular questions, the answers resulted in $25 \%$ of the total responses falling into this category. Participants highlighted the "importance to hear different inputs," but the final decision coming from the individual. One individual emphasized that "if you ask too many people for input, you will have too many answers." These responses emphasize keeping perspective and experiential maturity along with collaboration and self-motivation. Multidimensional leadership approaches involving reflection were observable in participant responses. 
"Reflection is essential, specifically, relating to the systems that have been put in place to ensure student achievement increases. When reflecting I will take a close look at the specific performance of individual students and teachers to know where the areas of needs are so that I devise a strategic plan.”

Round three also saw both 5\% of respondents reporting multidimensional approaches with an emphasis on the feminine (U/F) and another 5\% reflecting the masculine $(\mathrm{U} / \mathrm{M})$. In each of these instances, participants answered with a multidimensional approach; however, they chose to more so highlight the feminine or masculine approach in their answers. The following is an example of a masculine response that includes and underlying multidimensional undertone of keeping perspective $(M / U)$ : "I may not always ask for input from stakeholders in this area if what I am doing is working. I understand that the role of an administrator is not always to be loved, but to be respected. There will never be a time when $100 \%$ of the stakeholders will be happy with your actions, but we must remember that the students come first." Round three resulted in only $1 \%$ of responses categorized for the each of the subcategories of $\mathrm{M} / \mathrm{U}$ and $\mathrm{F} / \mathrm{U}$. These answers represented a major reporting category with undertones of multidimensional thought. Figure 3 illustrates the overall data for the third round. 


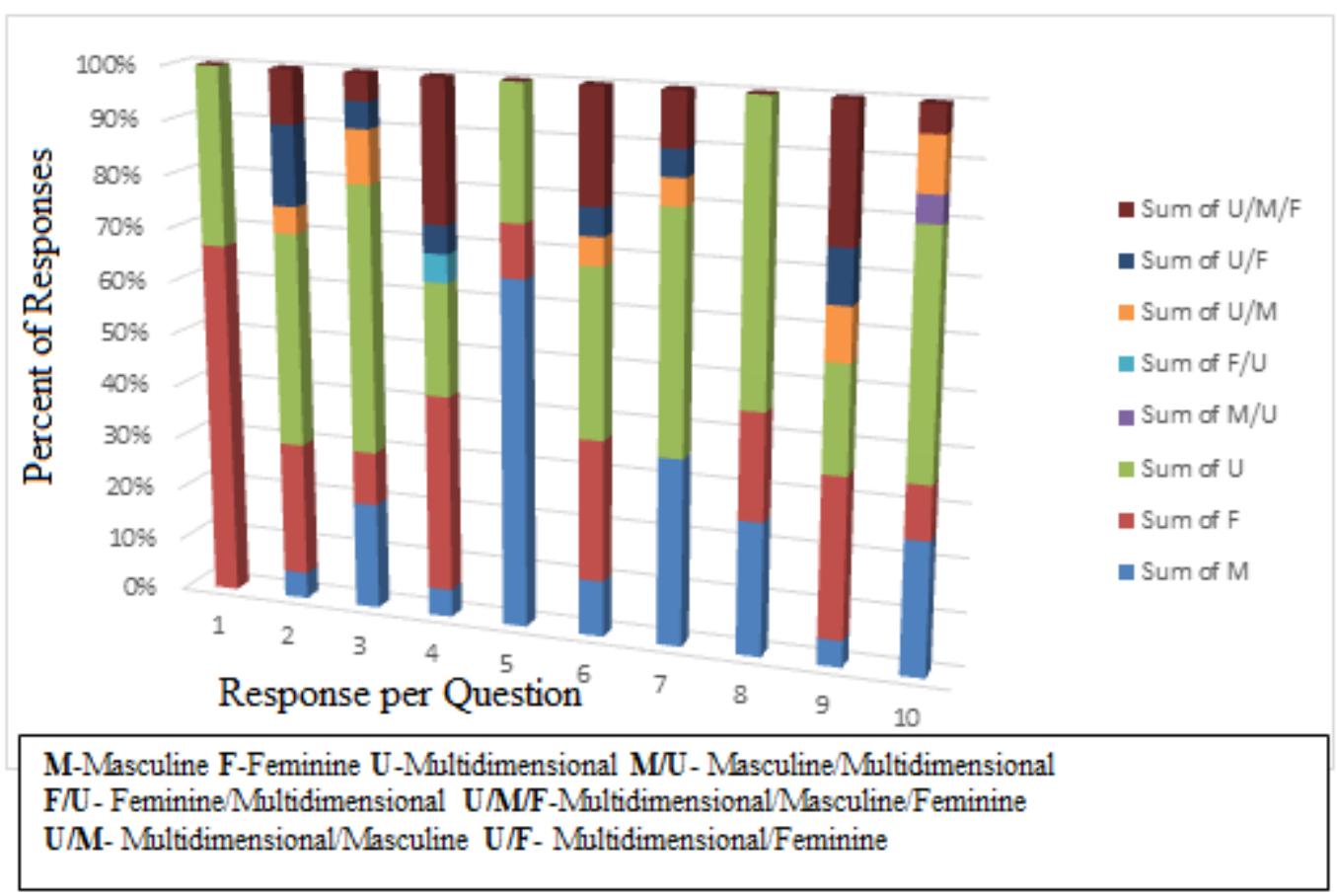

Figure 3 Round Three Overall

\section{Round Four}

As the only voluntary part of the study, round four's Final Reflections allowed participants the opportunity to see answer choices that were expressed for Round Three. Twelve of the 20 participants chose to provide input for this optional round. The results exhibited a solidification of the leadership profile for each participant. Additionally, the fourth round contributed to answering the research questions of the overall approach of current Miami-Dade County Public Schools leaders and how participants reported their decision-making processes.

The final reflections offered during the fourth round continued with a majority of multidimensional responses for leadership decision making, which resulted in $43 \%$ of answers provided by the participants representing this category. Nine of the 10 questions were dominated by responses that fell within the multidimensional category or 
subcategories; all questions except for number 6. Overall, styles that were expressed in these answers emphasized prioritizing, reflecting, and keeping perspective, which were evidenced by responses that showed the importance of "managing input" and "reflecting to ensure that time is used wisely."

"Priorities need to be analyzed. It is the ultimate goal for Administrators to participate in the tasks that are of high urgency and yield high results. Other tasks must be delegated to other stakeholders in order to keep the school focus and reach a successful goal. Delegating low priority and low urgency tasks is a MUST. Focusing on the responsibilities that are of high urgency and high importance are only possible when closely mapping and analyzing the tasks at hand. Prioritizing and organization are key."

Several participants stated that it is impossible to delegate all tasks, and knowing the capacity of key players plays a pivotal role in the sharing of responsibilities. Overall, there were more multidimensional answers that focused on a centered approach, rather than a masculine or feminine style to leadership. Feminine responses fell to its lowest rate of the study.

The number of responses in the feminine reporting category decreased from $20 \%$ of the total responses in round three to $14 \%$ of total responses in round four. Only one question was answered with this category as the most common leadership approach. This question involved the comparison of valuing family with the valuing of work priorities. Although $80 \%$ of participants chose the multidimensional approach in the survey question for round two's survey, the feminine approach of recognizing employees' family life over work life was expressed for question 7. Participants chose the significance of supporting 
individuals and being a role model to facilitate the nurturing of a specific culture within the school as reasons for expressing their leadership approaches. These traits happen to fall within the feminine leadership style.

"I believe in sympathy, empathy and compassion of what others are going through. I know that a lot of absences are a problem, but if this is an employee that is usually to work faithfully some special consideration should be given to that employee."

This participant has emphasized a belief in others. Even when considers that absences are a problem the reiteration of believing in the employee are maintained. Other feminine approaches that were unique to this round included improving morale amongst stakeholders, communicating needs, and teaming with individuals in a collaborative approach as the solution for overcoming obstacles. Masculine responses also fell to their lowest of the study with a $9 \%$ response rate.

Masculine approaches did not dominate any of the answers reported for round four with only minimal representation across all 10 questions. Participants abandoned emphasizing approaches that included failure not being an option, as well as taking an individual mindset to complete required tasks. Instead, participants highlighted more multidimensional and feminine responses that involved collaboration, team efforts, and optimism. In contrast to the masculine approach, there was an upswing in the representation of the subcategories for round four.

Round four was the first time that one or more subcategory dominated or tied a major reporting category for a question item. The subcategory $(\mathrm{U} / \mathrm{M} / \mathrm{F})$ resulted in $9 \%$ of total responses. This approach tied with two other categories/subcategories for one 
question. This question involved the delegation of tasks and ensuring accountability with follow-through to reach desired outcomes. Participants chose to emphasize the importance of "delegating tasks to subordinates... prioritizing work load" and "trusting but verifying" that tasks are being completed. Responses also emphasized the approach of keeping perspective, while utilizing both masculine and feminine styles to leading. The highest reporting subcategory in this instance was multidimensional/feminine (U/F).

Ten percent of responses reflected a multidimensional approach with an emphasis on feminine styles of leadership. This also tied the previous category in total responses on the same question about delegation and completion of activities. The participants that reported their leadership decision making within this subcategory highlighted teamwork and valuing others when completing tasks. These individuals congruently maintained the importance of keeping perspective and utilizing their experiential maturity. The subcategory of multidimensional/masculine (U/M) resulted in $8 \%$ of total responses by choosing to emphasize a similar approach. This contrasted a collaborative teamwork mindset with focusing on accountability and loyalty to vision instead. Table 2 conveys the overall leadership approach per participant throughout the study. 
Table 2 Participant Majority Reporting Category Across Rounds

\begin{tabular}{|c|c|c|c|c|}
\hline Participant & Round 1 & Round 2 & Round 3 & Round 4 \\
\hline Participant \# 1 & $\mathrm{~F}$ & $\mathrm{U}$ & $\mathrm{U}$ & $\mathrm{U}$ \\
\hline Participant \# 2 & $\mathrm{~F}$ & $\mathrm{U}$ & $\mathrm{F}$ & $\mathrm{U}$ \\
\hline Participant \# 3 & $\mathrm{~F}$ & $\mathrm{U}$ & $\mathrm{U}$ & $\mathrm{U}$ \\
\hline Participant \# 4 & $\mathrm{U} / \mathrm{M} / \mathrm{F}$ and $\mathrm{U}$ & $\mathrm{U}$ & $\mathrm{U}$ & $\mathrm{U}$ \\
\hline Participant \# 5 & $\mathrm{U}$ & $\mathrm{U}$ & $\mathrm{U}$ & $\mathrm{U}$ and $\mathrm{U} / \mathrm{F}$ \\
\hline Participant \# 6 & $\mathrm{U}$ & $\mathrm{U}$ & M & $\mathrm{U}$ \\
\hline Participant \# 7 & $\mathrm{U}$ & $\mathrm{U}$ & $\mathrm{U}$ & * \\
\hline Participant \# 8 & $\mathrm{~F}$ & $\mathrm{M}$ & $\mathrm{M}$ and $\mathrm{U} / \mathrm{M} / \mathrm{F}$ & $\mathrm{U}, \mathrm{U} / \mathrm{M}$, and $\mathrm{M}$ \\
\hline Participant \# 9 & $\mathrm{M}$ & $\mathrm{M}$ & $\mathrm{U}$ & M \\
\hline Participant \# 10 & $\mathrm{~F}$ & $\mathrm{U}$ & $\mathrm{U} / \mathrm{M} / \mathrm{F}$ & $\mathrm{U}$ \\
\hline Participant \# 11 & $\mathrm{U}$ & $\mathrm{U}$ & $\mathrm{U}$ and $\mathrm{M}$ & $*$ \\
\hline Participant \# 12 & $\mathrm{~F}$ & $\mathrm{U}$ & $\mathrm{U}$ & $*$ \\
\hline Participant \# 13 & $\mathrm{U}, \mathrm{U} / \mathrm{M} / \mathrm{F}$ and $\mathrm{U} / \mathrm{M}$ & $\mathrm{U}$ & $\mathrm{U}$ & $*$ \\
\hline Participant \# 14 & $\mathrm{U}$ and $\mathrm{U} / \mathrm{M} / \mathrm{F}$ & $\mathrm{U}$ & $\mathrm{U}$ & $*$ \\
\hline Participant \# 15 & $\mathrm{~F}$ & $\mathrm{U}$ & $\mathrm{U}$ & $\mathrm{U}$ \\
\hline Participant \# 16 & M & $\mathrm{U}$ & $\mathrm{F}$ & $\mathrm{U}$ \\
\hline Participant \# 17 & $\mathrm{M}$ and $\mathrm{F}$ & $\mathrm{U}$ & $\mathrm{U}$ & $*$ \\
\hline Participant \# 18 & $\mathrm{~F}$ & $\mathrm{U}$ & $\mathrm{U}$ & * \\
\hline Participant \# 19 & $\mathrm{M}$ and $\mathrm{F}$ & $\mathrm{M}$ & $\mathrm{U}$ & $*$ \\
\hline Participant \# 20 & $\mathrm{U}$ & $\mathrm{F}$ and $\mathrm{U}$ & $\mathrm{F}$ & $\mathrm{U}$ \\
\hline
\end{tabular}

* Participant did not participate in this Round

M-Masculine F-Feminine U-Multidimensional M/U- Masculine/Multidimensional

F/U- Feminine/Multidimensional U/M/F-Multidimensional/Masculine/Feminine

U/M- Multidimensional/Masculine U/F- Multidimensional/Feminine

Round four resulted in $6 \%$ of participants' responses with feminine approaches to decision making with underlying tones of multidimensional styles $(\mathrm{F} / \mathrm{U})$. A final reporting subcategory of masculine/multidimensional $(\mathrm{M} / \mathrm{U})$ resulted within a $1 \%$ of total responses. 
These individuals chose to focus on loyalty to mission, while acknowledging the importance of including key members of the leadership team in decision making. Data for the fourth round is illustrated in Figure 4. Additional tables conveying the data found during the course of this research are available in the Appendices section. Figure 5 conveys the results by leadership approach per round for the entirety of the study. Table 4 included in the Appendix V contains the participants' demographic information. Although this information was not paramount to the central questions in this research, this data is available for future research and verification of certain points that will be made during the discussion. A comparison of the overall results will be concluded in the summary section of the discussion chapter.

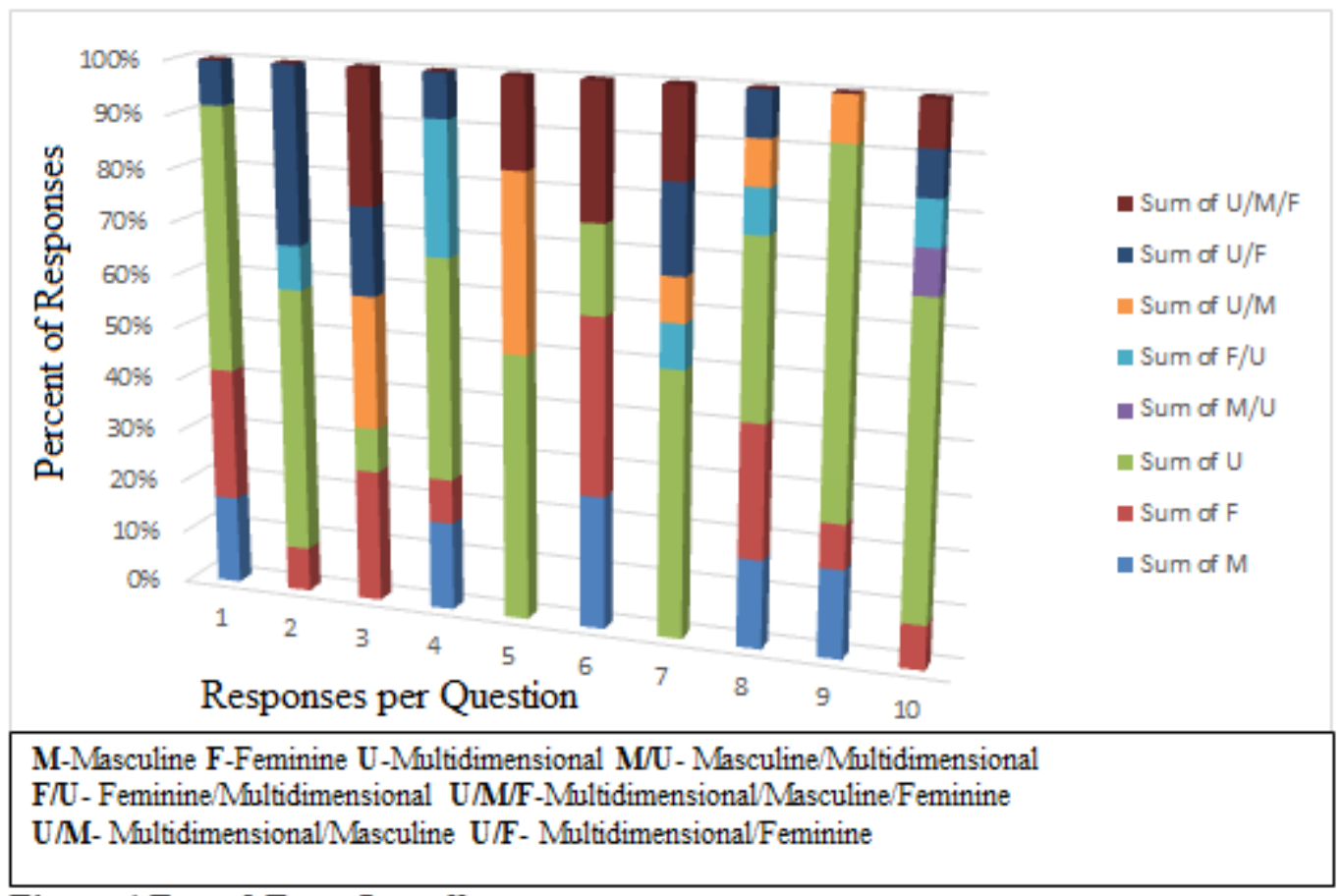

Figure 4 Round Four Overall 


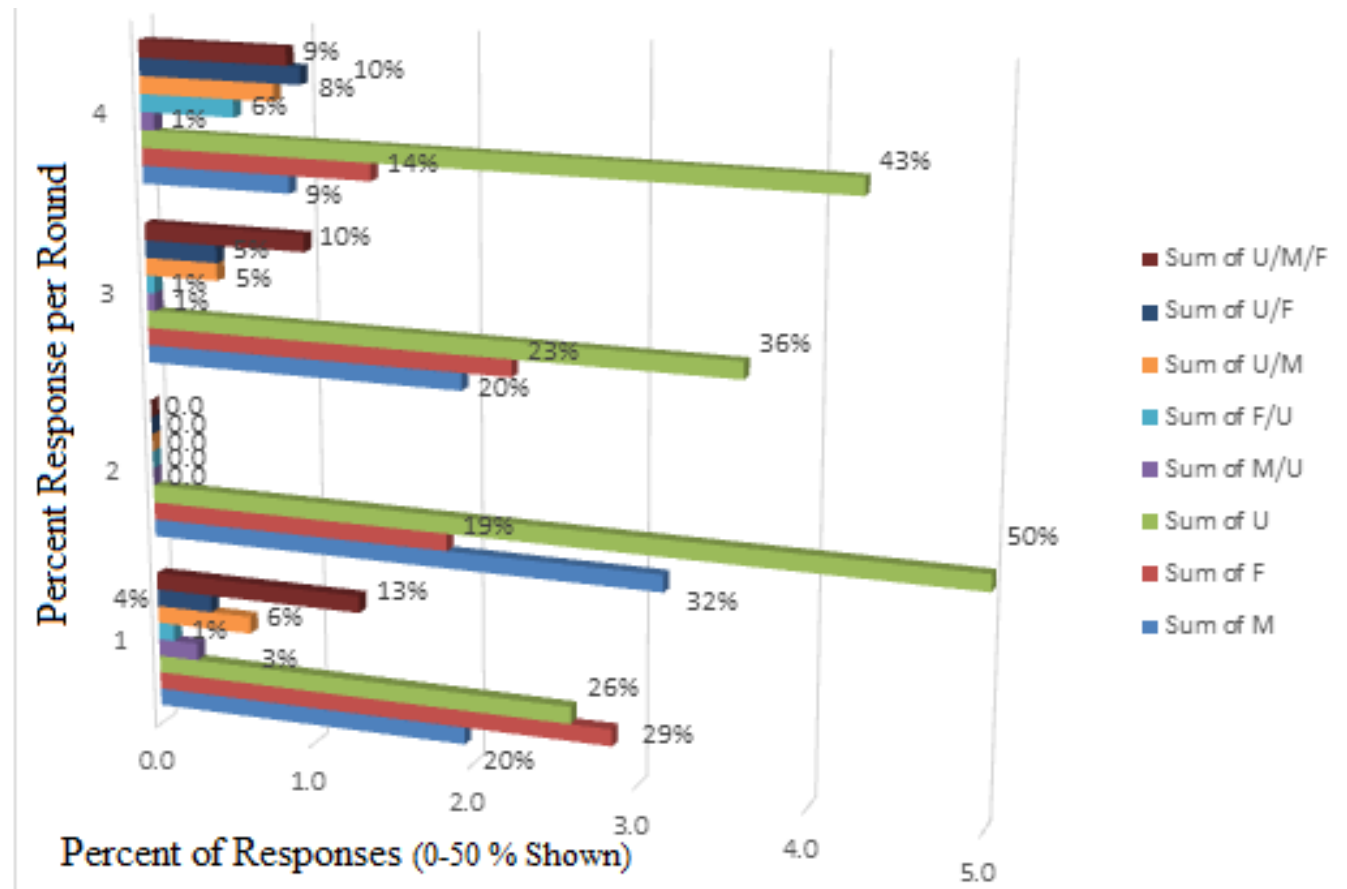

M-Masculine F-Feminine U-Multidimensional M/U- Masculine/Multidimensional F/U- Feminine/Multidimensional U/M/F-Multidimensional/Masculine/Feminine U/M-Multidimensional/Masculine U/F - Multidimensional/Feminine

Figure 5 Percentage Results by Reporting Category per Round 


\section{CHAPTER V}

\section{DISCUSSION}

This chapter serves as a summary for the study including an analysis involving the conclusions of the findings, a comparison with the extant literature. Also, the practical implications that may be considered upon a review of the results are discussed.

Additionally, recommendations for further research considerations are reviewed and presented.

\section{Summary of the Study}

This section will include three distinct segments: an introduction of the statement of the problem, a review of the procedures followed during the methodology, and a restatement of the guiding research questions. Following this section, a summary of the overall results of the study in relationship to the research questions will be discussed.

\section{Statement of the Problem}

With two-thirds of new leaders being women, it is becoming clearer that the face of our educational institutions is changing both symbolically and substantively (Chard, 2013; Currie, 2007; Danielson \& Schulte, 2007; Naidoo \& Perumal, 2014). The demands placed on public education are also growing exponentially. Schools are no longer isolated institutions that operate autonomously without immense external pressures. If society demands that schools become better, then it is necessary to explore school leaders' approaches to leadership. The literature has indicates that the decisions made by the school leadership are most associated with successful schools. 


\section{Statement of the Procedures}

Participation was solicited from a total of 20 individuals within Miami-Dade County Public Schools resulting in the participation of one regional administrative director, three principals, nine assistant principals, two instructional coaches, two district curriculum support personnel, one community involvement specialist, one team-leader, and one counselor. A modified electronic Delphi technique was implemented in four consecutive rounds, which was executed over the course of six weeks.

The first round asked the participants to answer an open-ended response 10-part questionnaire. An analysis of this data was used along with the supporting literature to create a survey for the second round. The survey presented specific situations with options that were stringently tied to a particular reporting category as the answer choices. The most common leadership approach found in responses was used to create the information presented in round three. Round three utilized a summary of the majority responses and offered them for the participants to reflect upon and/or revise their prior decision-making responses. A fourth and final optional round presented sample statements made by individuals in round three and asked the participants to make their final comments in regards to decision making with these statements in mind. What was presented in round four is available in Appendix Z.

\section{Statement of the Research Questions}

1) Based on the literature, what approaches of current Miami-Dade County Public School leaders may be understood to be masculine, feminine, or multidimensional? 
2) Based on the literature concerning masculine, feminine, and multidimensional leadership approaches, given information about other leaders' responses, how do Miami-Dade County Public School leaders report and explain their leadership decisions?

\section{Summary of the Findings}

Participants were able to express their decision-making processes by drawing on both experience and reason to answer questions and survey items. Rounds one, three, and four allowed individuals to respond in an open-ended format. Round two requested that participants choose the best decision amongst a pool of answers, where each reflected a certain reporting category approach in the response. This study resulted in the development of a snapshot of leadership approaches utilized by these school leaders. This section will summarize how the participants responded in regards to the reporting categories for each round. The following section will then utilize this evidence to answer the two research questions.

The number of participants who responded in round one with either masculine or feminine modalities as their leading reporting category outnumbered those who exemplified multidimensional thought. Of the 20 participants, 12 began the study with either a masculine or feminine approach in describing their overall leadership decisionmaking process. Eight of these individuals responded with dominance in the feminine category only. Two participants responded with masculine approaches alone as their dominant style. Two additional individuals had a tie between masculine and feminine. These participants had an equal number of questions resulting in masculine as they did 
with feminine responses, indicating that the individuals changed their approaches to decisions based on the circumstances presented. The remaining eight individuals in the study responded with multidimensional as their dominant leadership style. Round two resulted in an increase in participants reporting multidimensional approaches in their decision making.

Multidimensional approaches were observed among 16 of the studies participants for the Leadership Decision Making Survey. Out of the remaining four participants, three reported as masculine, while the last resulted in both feminine and multidimensional. The last required round, round three saw 16 participants' decision-making considerations turn towards multidimensional leadership approaches. One individual in the multidimensional category had the same representation of responses in the masculine approach category. Two of the multidimensional participants for this round resulted in the representation of multidimensional thought with the incorporation of both masculine and feminine approaches (U/M/F). One of these participant's responses also equaled the masculine category as his/her dominant reporting category. Three individuals were coded as masculine leadership as their dominant approach. Two participants reported their decision making and were analyzed to be feminine. The fourth round, although not required, offered another set of data that reinforced the dominance of multidimensional approaches.

From the 12 that participated in the final round, 11 of these were coded into the multidimensional category. Two of these 11 also reported their decision making that was equally reflective of other categories: one also was dominated by the subcategory of multidimensional/feminine (U/F) and both multidimensional/masculine (U/M) and masculine (M). Only one participant reported his decision making in a category other than 
multidimensional, which resulted in responses being coded in the masculine reporting category. Evidence of individual results is shown in Table 2 of the previous chapter. The findings indicated by the data were able to answer the two guiding research questions established at the onset of this study.

\section{Conclusions}

With regard to the first question of the type of approach that current school leaders utilize during decision making, it is clear that the reporting category that represented the majority of responses was multidimensional leadership. There was, however, a transition from participants reporting and explaining decisions with a more feminine approach to participant approaches representing a diverse array of leadership decision making. Following the initial round, the switch in mode amongst the analyzed data moved to multidimensional, which persisted for the subsequent rounds of three and four. The participants utilized the multidimensional approaches of keeping perspective, experiential maturity, prioritizing, optimism, and reflection to make decisions involving the institutions in which they lead. The majority of the evidence showed that these leaders implemented these approaches, rather than incorporating both masculine and feminine styles in their responses; however, the subcategories of U/M/F, U/M and U/F were present at different stages of the study. The second highest reporting category amongst participants was feminine leadership approaches.

Feminine leadership decision making was the leading reporting category for round one, which experienced a dramatic drop across the following rounds. This was more evident with the second round, which asked participants to make a decision in regards to 
specific situations with answer choices reflecting one of the major reporting categories. In this instance, no scenario item returned with a majority of participants choosing the feminine leadership approach. For round three, individuals were able to reflect on these majorities. Participants chose to emphasize the feminine aspect of decision making on three questions for round three that were previously identified in round two as multidimensional. By the summation of the study, participants no longer used feminine aspects of leadership alone to explain and report how they lead within their respective institutions. Masculine leadership was the lowest of the three major reporting categories.

Of the four participants that received a coding of masculine for the first round, only two of these individuals ended the study with the same approach as the majority in his/her decision-making thought process. One of these participants reported their decision making in the multidimensional category for the third round. After further and final reflections, this participant returned to the initial reporting category of masculine for the fourth round. The second research question of how do Miami Dade County Public School leaders report and explain their decision making has been answered in detailed through the discussion of the first research question; however, the following discussion will present final summary conclusions.

The majority of responses changed from initially feminine approaches in round one to multidimensional approaches by the end of the research. Of the 20 participants, half of these reported and explained their decision making with feminine approaches for round one. This reporting category saw the largest drop from an initial $50 \%$ of participants to no individuals utilizing the feminine reporting category approaches to expand on decision making in round four. Round three saw a limited resurgence of feminine attributes in 
describing leadership decisions with three participants coded as feminine leaders for this round. There was one participant who utilized multidimensional/feminine (U/F) approaches to report and explain his/her leadership thinking during the final reflection of round four; however, this individual also matched his/her total response with multidimensional approaches (U) as well. Overall, 19 of the 20 participants reported and explained their decision making with approaches from the multidimensional reporting category. Two of these participants also matched two or more of the categories or subcategories in their final responses. In one of these cases, the matching category was masculine approaches. Masculine leadership, therefore, ended with a total of two participants utilizing masculine specific approaches to report and explain their decision making.

In conclusion, a majority of participating educational leaders within Miami-Dade County Public Schools make decisions that are determined to be multidimensional in approach. School leaders in Miami-Dade County Public Schools report and explain making their decisions with the use of the attributes recognized by the supporting literature as multidimensional leadership approaches.

\section{Consistencies with the Literature}

The consistent reference to feminine leadership being strictly tied to women leaders makes approaching the discussion in this chapter more difficult. Christman and McClellan (2012) called this "a binary trap of viewing leadership through past and present social constructions of gender" (p. 64). The evidence from this particular study found that despite 16 of the 20 participants being women, there were no leaders who ended the study 
with a feminine leadership majority approach. In fact, feminine leadership reporting saw the largest drop out of all the reporting categories from 10 on the first round questionnaire, zero in the second round, two on the third, and back to zero again in the last round. Possible influencing factors will be discussed further in the implications portion of this chapter.

Exercising authority involves utilizing social capital earned by an investment in relationships. The findings in this research illustrated that a large majority of school leaders in Miami-Dade County Public Schools invest in relationships. They categorize family as the number one priority and intend to create an atmosphere that is supportive and caring. These individuals are better able to build social capital by developing these connections. Practicing appropriate leadership involves understanding how to exercise interpersonal skills along with emphasizing of task-oriented objectives.

Lewin and Regine (2003) described this person as a third possibility leader who is beyond the confines of the gender dichotomy. A third possibility leader is simply another term for androgynous leader. Multidimensional leaders are third possibility leaders, because they break traditional norms and refuse to follow either biological sex-roles or perceived gender-roles. Participants in this study described their decision making in mirroring terms of the literature by being a "whollistic thinker" with a deep understanding of a multi-linear approach to management (Lewin \& Regine, 2003, p. 350). The participants exhibited this behavior by emphasizing the attributes found in the multidimensional reporting category as shown in Table 1. Lewin and Regine (2003) also argued that third possibility leaders build trust, which was a relevant approach that was expressed amongst the multidimensional decision makers in the study. Although not 
significant to the findings of this research, the emphasis on the sex of the individual leaders within the literature provides some explanation for the understanding of traditional viewpoints on men and women as leaders.

Christman and McClellan (2012) acknowledged that both men and women face different barriers defined and influenced by social constructs within society. Many of these boundaries are due to the traditional view of leadership that is rooted in a masculine view-point; however, even those male leaders who incorporate feminine approaches into decision making face a fear being labeled as "weak and submissive" (p. 662). Kloot (2004) provided that one of the greatest threats to women adopting masculine traits is being viewed as inauthentic. Authenticity is a key element of appropriate leadership. Leaders fear being perceived in a way that will lead to their rejection by stakeholders and/or the ultimate failure of the organization. This provides argument for the need of leaders to incorporate self-reflection into their everyday practice (McNamara \& O'Hara, 2008). Only then can the individual adopt an adaptive style that offers the appropriate leadership approaches needed for each situation. Some of the research is consistent with the findings of this study by providing an invitation to go beyond the sex of the leader and unveil true depictions of what constitutes appropriate leadership approaches in complex environments.

Chistman and McClellan (2012) have argued that the gender-oriented approaches are no longer static. This research also reflected that the sex of the participant did not determine what the individual's overall leadership approach. Therefore, the support for pushing the tone of conversation passed sex-orientation stereotypes has reached a crucial point. This research asks us to go beyond the sex of the individual leader. 
It pushes one to reject gender-role assignments based on archaic viewpoints based on biological stereotypes and traditional norms.

Coleman and Pounder (2002) referred to leaders who offer a balanced leadership approach as androgynous. A majority of participants exhibited an androgynous style of leadership during the course of this study. Evident in the findings, the relationship between androgynous and multidimensional leadership can be synonymous in terms of reflection, experiential maturity, and keeping perspective.

Oplatka (2015) argued that constituents want leaders that stress both positivefriendly relationships and emphasize egalitarian qualities in organizations (p. 48). Although this "feminine sex-role orientation" is a desire of stakeholders for leadership, it is expected that these individuals have the authority to reprimand and punish when necessary (p. 52). Multidimensional leaders in this study emphasized holding people accountable and following-through on commitments. While typically a masculine approach and an important component of organizational leadership, multidimensional participants did not refer to enforcing strict accountability as their primary objective. Instead, these leaders highlighted inviting individuals into the decision-making process. This paradoxical approach is androgynous in nature and ultimately seen as multidimensional. Oplatka (2015) later concluded that stakeholders prefer an androgynous style of leadership, but the special needs of each school may differ (p. 53). These differing circumstances beg for the leader to determine the most appropriate leadership approach that will benefit his/her particular school. 


\section{Inconsistencies with the Literature}

As mentioned previously, much of the literature is fixated on the idea of sexidentification and the gender approaches associated with these labels. The emphasis of sex-identification is evident by the automatic allocation of feminine attributes with female leadership and masculine attributes to male leadership. Marinescu, Saseanu, and Toma (2014) stated that women "have the tendency to adopt" certain feminine styles of leadership. The expectation is that women would represent the majority of feminine leadership and feminine leadership reporting. This was not the case during the course of the study, as feminine leadership was not strictly limited to those participants who were female. Although the initial reporting of decision making did have more feminine responses in the first round, the feminine reporting category never reached majority numbers again throughout the study.

The literature relies on associating certain approaches with women leaders rather than recognizing that the connection between sex and gendered approach is not stringently tied together. The current research study illustrates that regardless of the participant's sex, leadership approach is determined by a confounding of factors. Kruger (2008) called the identification of certain approaches with a specific gender as gender-role associations.

Ayman and Korabik (2010) argued that leadership research is not a "genderneutral phenomenon" (p. 165); however, they did confess that stereotypical behaviors of each sex might be related to cultural values rather than simply biology. This idea supports Eckman's (2004) contention that leadership style is determined by both biological and environmental factors. Leaders may not be able to easily change their particular sex, but as creatures of free will, decision making must be a pragmatic process that involves self- 
reflection and full understanding of the consequences of certain actions (Ozga, 2009). Although four of the 20 participants in this study were male, only one of them began and ended the study classified as a masculine decision maker. Coincidently, 15 women ended the study with a majority of markers in the multidimensional leadership approach, as well as with many responses representing a spread across the different reporting categories and subcategories.

Kloot (2004) stated that women do not lead differently, but are only "perceived as different" (p. 472). Although most of the literature does speak to this particular argument, it may give some insight in to why certain expectations were not matched by the results found in this research. Evidenced by the number of participants in this study that exhibited multidimensional decision making, perhaps a new era in research is required to understand aspects of organizational leadership. This new era may be even more so essential for the leadership dynamics now emerging in our public schools.

Miami-Dade County Public Schools is the fourth largest school district in the United States with a diverse student population. These students come from countries from across the globe, and represent multiple socioeconomic classes, ethnicities, and certainly cultures. With a majority of participants reporting and explaining their decision-making processes by use of a multidimensional approach, it is important to support increased research in this area.

\section{Limitations}

Participants were sought through district and region meeting announcements, email invite, and one-to-one conversation by the researcher. The major limitations in this 
study are centered on three distinct areas represented by the following questions: first, Are those who would volunteer to participate in a dissertation study more likely to incorporate alternate forms of decision making into their practice?, second, What are the consequences associated with the job position titles of participants and the ultimate decision making that takes place at a school?, and third, Does a Delphi technique methodology lend itself to findings that are more multidimensional?

In regards to the first question, the implication that those who volunteered for the study may be more multidimensional can only be speculative. It can be assumed that school leaders who hold a titled position are likely more ambitious and willing to take on the responsibilities incorporated with involvement in education research. Neither the supporting literature nor the data obtained during the course of this study offers a clear answer; however, this question may require further research and clarification in order to decrease its limitations on interpreting the findings. The researcher attempted to account for the second question and limitation relating to job title by including as many top-level administrators and even a regional director in the research process as possible.

It proved more difficult to get through to principals at schools even after they had committed to the study. There was certainly a figurative wall protecting these individuals from persons outside the recognized stakeholders that was not easily penetrated. This made communication with these individuals more difficult. This resulted in fewer principals participating in the study than was expected. Even though the research supports the primary notion that decision making involves a spider-web of influence, which was one of the foundational notions to this research, principals make the ultimate decisions at the school level (Fullan, 2001). Although a limitation, the data obtained during the course 
of this study does represent the many layers surrounding decision making within public schools. A third foreseeable limitation is the impact of a multi-round study on the outcome of the research.

A Delphi technique allows the researcher to investigate a phenomenon by exploring theories through the interactions among participants. The researcher was able to investigate how these particular school leaders utilized different approaches in the leadership decision-making process to accommodate the needs of their schools. The third question involving the influence of this interaction on the outcomes creates a possible limitation. The participants have their particular views, leadership styles, and decision making principles. When the individuals interact and the thoughts of others are known, the participant is asked to be reflective.

Reflection is a multidimensional leadership action. It is assumed that the individuals will utilize the best-perceived decision-making approach to answer proceeding questions and survey items; however, there may be a level of influence that impacts the study. In this particular study, the data conveyed a move to multidimensional leadership decision making, which brings into account two additional questions: Is there a difference between how a participant reports his/her decision making versus how they actually make decisions?, and, Did the interaction that took place during the Delphi technique lead to the data showing more multidimensional leaders?

The first round saw the majority of responses in the feminine reporting category. When participants were asked to explain their leadership style or decision-making process in open-ended form in rounds three and four, the majority answered in a multidimensional style. It was, however, in these three specific rounds that the participants expressed their 
styles by using the other various reporting categories as well. This is particularly true with feminine leadership styles, which fluctuated tremendously throughout the study. Participants readily expressed their belief in others and the value that they placed in individual contribution to the success of the organization. However, the abandoning of the feminine leadership approach in the second round's decision making may indicate something larger that is worthy of further research.

The Decision Making Survey of round two asked participants to choose the best decision for each particular scenario. In these particular cases, individuals chose multidimensional approaches a majority of the time. Masculine leadership decision making was also represented, which offers more intrigue into the consideration of the first question of whether individuals report and explain their decisions in contrast to the decisions they actually make. This intrigue should lead to further research into the ideals of leaders versus the reality of leaders. In other terms it brings to the forefront is the espoused versus actual expectations that leaders operate by. More specifically, is there a difference between the idealistic perspective of how leaders make decisions and what situational decision making actually takes place? The second question about the influence of the Delphi methodology cannot easily be answer, but certain aspects can be observed and reported.

There are key factors to report in regards to this pending question and potential limitation. The first being the difference in how each participant explained and then actually made decisions in the second round Situational Decision Making Survey. This, of course, is described above. The participants then return to explaining their decisionmaking process by using a diverse array of reporting categories. The results conveyed that 
the majority responses remained in the multidimensional category and this reporting saw the greatest amount of representation throughout the duration of the study. But whether the multi-step and interactive nature of the Delphi technique used in this study overtly influenced participants is not overtly clear.

Although the multidimensional approach received the most support compared to the other two major reporting categories with an end result of $43 \%$ of participant responses measured to $26 \%$ participant of responses at the start, the rest of the subcategories remained relatively stagnant. It can be inferred that the interaction and reflective nature may have impacted the participants by swaying their responses from representing entirely masculine or wholly feminine towards either a more reflective or multidimensional response. This may lead us to the question, what if the majority of the participants had overwhelming expressed their decision making in one category such as the masculine or feminine persistently throughout the study? Would this lead to more participants adopting these ideas as their own by the end rounds of the research? This can only be answered by further research. The final limitation already detailed in Chapter III involves the sample size.

The mixed methods approach relied on the input from school leaders to give responses involving leadership approaches. Further researcher with increased sample sizes utilizing the findings to the present study will strengthen the significance and generalizability of the results. 


\section{Implications for Practice}

The prima facie leaders of schools are the administrative staff. This begins with principals and assistant principals, who delegate the responsibilities of specific tasks down through a chain of command. This hand-off of power can be in the form of shared leadership as seen through a power-with or as a power-over (Northouse, 2013). The evidence conveys that effective schools adopt the power of community by empowering each individual to align collective efforts toward the overall vision and mission of the organization. This discourse intends to identify the approaches of leaders in schools through the details that surround management and leadership.

Management and leadership play a significant role in organizations, as the management of people often involves many transactional events. Previously identified as a masculine characteristic, the relationship between supervisor and subordinate is a check on roles and responsibilities (Northouse, 2013). The inverse is a more feminine approach referred to as transformational leadership. These interactions involve a level of growth with both supervisor and subordinate as they strive to make gains towards set goals together (Young, Mountford, \& Skrla, 2006). The end result is both individuals mutually transforming by both the impact of their relationship and the process of making strides together. This research identifies multidimensional leadership as a major proponent of how some school leaders make decision. However, it also exhibits the importance of transactional relationships as a significant part of the decision-making process.

The requirement of school leaders to utilize both transactional and transformational forms of management and leadership is an essential paradox. The supporting literature has identified this contradictory notion. The research surrounding 
multidimensional leadership are seemingly ambiguous and paradoxical; however, this research offers insight into how a multidimensional approach by useful to both current and aspiring leaders. It is the practices seen in the methods of self-reflection that allows school leadership to fully realize the most appropriate approaches (Beebe \& Nogay, 2008). Determining factors often incorporate the environment of the school, including its history and culture, as well as current pending factors. The style of a leader can be seen through a scale of masculine approaches on one end and feminine on the other.

The masculine and feminine leadership paradigm requires contemporary leaders in schools to not simply identify with the approaches of males or females, but to dissect their management and leadership style in a multidimensional way (Scott \& Brown, 2006). To simply study the sex of school leaders and make conclusions about the effect of each depending solely on their biological attributes would be to neglect the complex and paradoxical nature of school leadership. This would also fail to take into consideration the different approaches adopted by both sexes to accommodate the needs that are foreseeable within the organization (Reynolds et al., 2008). The literature presented has already recognized that both men and women adopt attributes of both masculine and feminine styles to accommodate the needs of their respective organizations. The multidimensional approaches required of school leaders must incorporate an array of characteristics as this infuses a balanced interpretation of what schools need (Brown \& Light, 2012). The decisions of multidimensional leaders are formed through a reflective process that not only understands the needs of the organization, but also realizes the relationship between leadership approaches and their influences on the school as an organization. 
The efforts put towards reformative change in schools are at the mercy of the type leadership culture fostered by decision makers. This culture of leadership defines the environment of how power and resources alike are distributed in the organization. Schools will either rise or fall according to the characteristics of leadership that are practiced. At the ground level, the role of the principal has been evolving and has been identified as one of the most important and influential positions in schools seeking to improve their education practices (Hallinger \& Heck, 1998). As previously established, leadership goes beyond the realms of the office of the principal within a school. As leaders tackle the challenges of instituting reformative change within each school, it is their responsibility and within their power to stimulate, motivate, and inspire (Eilers \& Camacho. 2007). By accepting the influence that positive school management has and by further defining what constitutes appropriate leadership, public school leaders will be able to formulate a set of approaches that leads to greater student achievement.

Our educational institutions are diverse and complex and the demands placed on them require leaders not only to know the characteristics of appropriate leadership, but also to be intimately aware of their own leadership styles. The development of an individual's leadership approaches should be an evolving discipline that is consistently revisited through mindful research and practice. School leadership will be able to best accommodate the needs of the diverse environments in which they traverse by involving the processes surrounding self-reflection. This research suggests that mindful practice allows for individuals to curtail their individual paths towards appropriate leadership and the ability to make decisions that may positively impact the organizations in which they lead. 


\section{Implications for Research}

The present study conveyed that school leaders utilize multidimensional approaches to make decisions: they self-reflect in order to formulate the most appropriate decision-making process. The current research highlights the idea of appropriate leadership and the determination of this factor relying on the circumstances in each school. Appropriate leadership requires self-regulation and leads to a more flexible organization. Appropriate leadership can adapt to challenges and be assertive when confronted with adversity. This is a necessity for the continued growth of school leaders and leadership across public schools (Ayman \& Korabik, 2010). This will also contribute to the ability of schools to remediate steps taken and revise the overall methods implemented to reach greater effectiveness. Schunk (2012) suggested that self-regulation helps support the initiatives of school leadership, which has been found to be an inherently multidimensional leadership style.

The current study has presented those who use experiential maturity and perspective as qualities of current school leaders. They utilize these multidimensional approaches to offer an appropriate approach to the challenges facing public schools. Emerging leaders must go beyond a binary gender-specific style to adopt the attributes of androgynous and third possibility leaders. The processes of self-regulation will force school leadership to take seriously the idea of an appropriate approach to leadership. Appropriateness can only be determined in each circumstance by the needs presented by the setting and environment at a particular school (Kruger, 2008).

Individuals who wish to become leaders in education and contribute to the success of the organizations in which they work may look to the present study and the relevant 
supporting literature for guiding principles in developing appropriate leadership skills. These persons have the added benefit of looking at top-level decision makers and adopting their approaches and styles to combat the growing complexities facing public education today (Hyle, Ivory, \& McCellan, 2010). Remediation is taken when approaches do not reach favorable outcomes. Third possibility and androgynous leaders pursue a multidimensional approach that goes beyond perceived sex-roles, gender-specific styles, and the norms established by history and tradition to deliver the type of leadership that stakeholders are demanding at that moment in time.

The data revealed that school leaders motivate and inspire members of their organizations by utilizing the attributes of multidimensional leadership was also congruent with the literature (Cardelle-Elawar, Iriarte, de Sanz \& Ugarte, 2003, p. 60). Self-reflective activities support decision making by practicing the methods of selfreflection and becoming a "support mechanism" for the culture within an institution (McNamara \& O'Hara, 2008, p. 176). Even though the language that dominates the literature in the field of leadership and public education involves the notion of determining effective leadership, the present research implies that effectiveness relies on the appropriateness of leadership style that is determined by the needs of the environment.

\section{Suggested Further Research}

This study was able to answer the guiding research questions along with the majority of questions that were developed during the course of the research. Consequently, some questions were highlighted leaving the relationship between certain 
conclusions and implications ripe for further efforts of discovery. It also became apparent for the need of further research in this field throughout this discussion. The following are the recommendations for further research in order to expand upon the findings presented in this study.

1) Is there a correlation between leadership title and the type of leadership approach that is utilized?

2) Is there a negative correlation between how school leaders describe their ideal decision-making process with the actual decisions made under-pressure during situations that present adversity?

3) Replicating this study in both like and unlike school districts would strengthen the generalizability of its findings. The question of what leadership approaches are practiced within small or large school districts would be answered as well as offering an opportunity to determine if multidimensional leadership has created a new norm or expectation of current school leaders.

4) This research can and should also be replicated in settings beyond education, as leadership study is often generalized across many fields of organizational management and leadership.

5) Including the demographics of participants in similar research studies could determine whether themes in past literature are relevant to the rapid changes caused by the complexities in today's society. As mentioned within this chapter, the majority of peer-reviewed scholarly literature presents the approaches of leaders in terms of the sex of the individual by making bold and broad sweeping statements such as "men tend to do" and "women tend to do." The field of 
educational leadership and especially organizational leadership is begging for the research to go beyond the confines of biology to ultimately determine what comprises the decision making in providing the appropriate type of leadership approach based on circumstance and setting.

6) The research is also centered on effective leadership; however, the majority of individuals will state that the best decision is dependent upon specific and certain circumstances rather one set of approaches that works in each setting. More research is needed to determine what constitutes appropriate leadership and how to develop the skill of determining the most appropriate approach in relationship to the setting and environment of the leader.

\section{Summary}

The discussion provided in Chapter 5 offers a summary of the study by presenting the statement of the problem, the procedures followed, and the guiding questions. The findings found that school leaders at many levels in Miami-Dade County Public Schools utilize a multidimensional approach to decision making. They also use terms that are not reflective of any particular gender, which is represented in the supporting literature as androgynous or third possibility leadership. While multidimensional leadership represented the majority of responses, school leaders still infused masculine and feminine approaches at certain times according to the situations that unfolded.

These findings suggest that leaders must know their environments. This mindful and reflective practice includes recognizing the needs and desires, history and cultures, as well as the stakeholders within the organizations that they lead. Organizational leaders 
would benefit from an awareness of how their actions influence the objectives and the stakeholders within their respective institutions. Individuals with the ambition to tackle the challenges of leadership responsibility must willingly accept that a clear-cut picture of what constitutes effective leadership is nonexistent and the consolation must be found in determining the most appropriate leadership approach. Some schools may benefit from one approach over the other, which is to be determined by history, culture, constituency, and circumstances unique to the organization.

Coleman and Pounder (2002) contended that the arguing for either feminine or masculine over the other "misses the point" in defining effective management and leadership. The methodology outlined during the course of this study allowed the researcher to explore the feminine, masculine, or multidimensional leadership approach of school leaders. Additionally, allowing for participants to reflect and respond to the statements of others echoes the relevant themes in the field of research surrounding school leadership. The literature suggests that multidimensional leadership is promoted by blending masculine and feminine forms of leadership through "intense self-reflection" and the "development of self-awareness and identity" (Christman \& McClellan, 2012, p. 651). School leaders should be cognizant of their decision-making processes and utilize a leadership style that is mindful of the needs within each particular school. 


\section{REFERENCES}

Anderson, R. C., \& Klofstad, C. A. ( 2012). Preference for leaders with masculine voices holds in the case of feminine leadership roles. PLOSone, 7(12), 1-4.

Ayman, R. and Korabik, K. (2010). Leadership: Why gender and culture matter. American Psychologist, 65(3), 157-170.

Barnett, K. and McCormick, J. (2004). Leadership and individual principal-teacher relationships in schools. Educational Administration Quarterly, 40(3), 406-434.

Beachum, F. D. and McCray, C. R. (2010). An analysis of how the gender and race of school principals influences their perceptions of multicultural education. International Journal of Education Policy \& Leadership, 5(4), 1-10.

Beebe, R. J. and Nogay, K. (2008). Gender and perceptions: Females as secondary principals. Journal of School Leadership, 18, 583-602.

Bolman, L. G. and Deal, T. E. (2008). Reframing organizations: Artistry, choice, and leadership. San Francisco, CA: John Wiley \& Sons.

Brown, S., \& Light, R. L. (2012). Women's sport leadership styles as the result of interaction between feminine and masculine approaches. Asia-Pacific Journal of Health, Sport and Physical Education, 3(3), 185-198.

Burns, J. (1978). Leadership. New York, NY: Harper \& Row.

Calvanese, M. (2007). Investigation gender stereotypes in elementary education. Journal of Undergraduate Psychological Research, 2, 11-18.

Camacho, A \& Eilers, A. M.,. (2007). School culture change in the making: Leadership factors that matter. Urban Education, 42(6), 616-637.

Chard, R. (2013). A study of current male educational leaders, their careers and next steps. Management in Education, 27(4), 170-175

Cheng, Y. C. (1994). Principal's leadership as a critical factor for school performance Evidence from multi-levels of primary schools. School Effectiveness and School Improvement, 5(3), 299-317.

Cheng, Y. C. \& Shum, L. C.. (1997). Perceptions of women principals' leadership and teachers' work attitudes. Journal of Educational Administration, 35(2), 165-184.

Christman, D. E., \& McClellan, R. L. (2012). Discovering middle space: Distinctions of sex and gender in resilient leadership. Journal of Higher Education, 83(5), 648670. 
Coleman, M., \& Pounder, J. S. (2002). Women-Better leaders than men? In general and educational management it still "all depends." Leadership \& Organization Development Journal, 23(3), 122-133.

Currie, T. (2007). New leaders for troubled schools: Jacquelyn Davis works with D.C.'s education bureaucracy. Education Next, 7(1), 28-34.

Dahlvig, J. E. (2013). A narrative study of women leading within the council for Christian colleges \& universities. Christian Higher Education, 12(1), 17.

Danielson, L., \& Schulte, L. (2007). Voices of women in the field: The top 10 things we learned about being a department chair. Journal of Women in Educational Leadership, 5(1), 77-81.

Donaldson, G. A. (2006). Cultivating leadership in schools: Connecting people, purpose and practice (2nd ed.). New York, NY: Teachers College Press.

Eckman, E. W. (2004). Does gender make a difference? voices of male and female high school principals. Planning and Changing, 35(3), 17.

Evans, A. E. \& Reed, L. (2008). 'What you see is [not always] what you get!' Dispelling race and gender leadership assumptions. International Journal of Qualitative Studies in Education, 21(5), 487-499.

Ford, J. (2005). Examining leadership through critical feminist readings. Journal of Health Organization and Management, 19(3), 236-251.

Fullan, M. (2001). Leading in a culture of change. San Francisco, CA: Jossey-Bass.

Gelberg, D. (2007). The business agenda for school reform: A parallel universe. Teacher Education Quarterly, 34(2), 45-58.

Goodman, R. H., \& Zimmerman, W. G., Jr. (2000). Thinking differently: Recommendations for 21 st century school board/superintendent leadership, governance, and teamwork for high student achievement. Arlington, VA: Educational Research Service.

Guramatunhu-Mudiwa, P. \& Bolt, L. L. (2012). Does the gender of school personnel influence perceptions of leadership? School Leadership and Management. 32(3) 261-277.

Hallinger, P., \& Heck, R. H. (1998). Exploring the principal's contribution to school effectiveness: 1980-1995. School Effectiveness and School Improvement, 9(2), 157-191. 
Hsu, C. \& Sandford, B. (2007). The Delphi technique: making sense of consensus. Practical Assessment, Research \& Evaluation, 12, 1-8.

Hyle, A. E., Ivory, G., \& McClellan, R. L. (2010). Hidden expert knowledge: The knowledge that counts for the small school-district superintendent. Journal of Research on Leadership Education, 5(4), 154-178.

Jang, Y. S. , Ott, J. S. , \& Shafritz, J. M. (2011). Classics of organization theory. Boston, MA: Wadsworth, Cengage Learning.

Johnson, C. (2012). Meeting the ethical challenges of leadership: Casting light or shadow. Los Angeles, CA: Sage.

Kirst, M. W. \& Wirt, F. M. (2009). The political dynamics of American education. Richmond, CA: McCutchan.

Kloot, L. (2004). Women and leadership in universities: A case study of women academic managers. The International Journal of Public Sector Management. 17(6), 470-485.

Kruger, M. L. (2008). School leadership, sex and gender: Welcome to difference. International Journal of Leadership in Education, 11(2), 155-168.

Leithwood, K., \& Jantzi, D. (2008). Linking leadership f student learning: The contributions of leader efficacy. Educational Administration Quarterly, 44(4), 496-528.

Lewin, R. \& Regine, B. (2003). Third possibility leaders: The invisible edge women have in complex organizations. The Learning Organization. 10(6), 347-352.

Marinescu, P., Saseau, A., \& Toma, S. (2014). Feminine leadership and oganisational culture. Leadership Prospects. 19, 144-150.

Maslow, A. H. (1991). Critique of self-actualization theory. Journal of Humanistic Education and Development, 29(3), 103-108.

McNamara, G., \& O'Hara, J. (2008). The importance of the concept of selfevaluation in the changing landscape of education policy. Studies in Educational Evaluation, 34(3), 173-179.

Moreno, M. S. \& Yanez, J. L. (2007). Women leaders as agents of change in higher education organizations. Gender in Management: An International Journal. 23(2), 86-102. 
Murphy, J. (2005). Connecting teacher leadership and school improvement. Thousand Oaks, CA: Corwin Press.

Naidoo, B., \& Perumal, J. (2014). Female principals leading at disadvantaged schools in Johannesburg, South Africa. Educational Management Administration \& Leadership, 42(6), 808-824.

Northouse, P. G. (2013). Leadership: Theory and practice. Thousand Oaks, CA: Sage Publications.

Oplatka, I. (2006). Women in educational administration within developing countries: Towards a new intentional research agenda. Journal of Educational Admininstration. 44(6), 604-624.

Owen, M. A. (2010). Creating a successful professional culture: Reorganizing to rebuild a challenging school. Journal of Cases in Educational Leadership. 12(4), 110 .

Ozga, J. (2009). Governing education through data in england: From regulation to self evaluation. Journal of Education Policy, 24(2), 149-162.

Reed, L. C (2012). The intersection of race and gender in school leadership for three black female principals. International Journal of Qualitative Studies in Education, 25(1), 39-58.

Reynolds, C., White, R., Brayman, C., \& Moore, S. (2008). Women and secondary school principal Rotation/Succession: A study of the beliefs of decision makers in four provinces. Canadian Journal of Education, 31(1), 32-54.

Riches, C. \& Morgan, C. (1989). Human resource management in education. Open University Press, Milton Keynes.

Sanz de, A. L., Ugarte, D. M., Cardelle-Elawar, M., Iriarte, D. M., \& Sanz de, A. B. (2003). Enhancement of self-regulation, assertiveness, and empathy. Learning and Instruction, 13(4), 423-439.

Sahin, S. (2011). The relationship between instructional leadership styles and school culture. Eudcational Sciences: Theory \& Practice, 11(4), 1920-1927.

Sawyer, M. R. (2000). The black church and black politics: Models of ministerial activism. In Down by the riverside: Readings in African American Religion. Ed. L. Murphy. New York, NY: NYU Press.

Scott, K. A., \& Brown, D. J. (2006). Female first, leader second? gender bias in the encoding of leadership behavior. Organizational Behavior and Human Decision Processes, 101(2), 230-242. 
Schunk, D. H. (2012). Learning theories: An educational perspective (6th ed.). Boston, MA: Pearson Education.

Sergiovanni, T.J. (1984). Leadership and excellence in schooling, Educational Leadership, 41(5) 4-13.

Sergiovanni, T.J. (2009). The principalship: A reflective practice perspective (6th ed.). Boston, MA: Allyn \& Bacon.

Skrla, L, Young, M., \& Mountford, M.. (2006). Infusing gender and diversity issues into educational leadership programs: Transformational learning and resistance. Journal of Educational Administration, 44(3), 264-277.

Taleb, H. M. (2010). Gender and leadership styles in single-sex academic institutions. International Journal of Educational Management, 24(4), 287-302.

Vladero, D. (2009) Women on par with men in principalship, says report. http://blogs.edweek.org/edweek/inside-school research/2009/07/women_on_par_with_men_in_princ.html. (July 1, 2009)

Waters, T. W., \& Cameron, G. (2007). The balanced leadership framework: Connecting vision with action. Denver, CO: Mid-continent Research for Education and Learning. 


\section{APPENDIX A}

TABLE 3. Response Majority by Round per Question

\begin{tabular}{|c|c|c|c|c|}
\hline Question \# & Round 1 & Round 2 & Round 3 & Round 4 \\
\hline 1 & $\mathrm{~F}$ & $\mathrm{U}$ & $\mathrm{F}$ & $\mathrm{U}$ \\
\hline 2 & $\mathrm{~F}$ & $\mathrm{U}$ & $\mathrm{U}$ & $\mathrm{U}$ \\
\hline 3 & $\mathrm{U}$ & $\mathrm{U}$ & $\mathrm{U}$ & $\begin{array}{c}\text { (Three-way tie U/M/F, } \\
\text { U/M, F) }\end{array}$ \\
\hline 4 & $\mathrm{U}$ & $\mathrm{U}$ & $\mathrm{F}$ & $\mathrm{U}$ \\
\hline 5 & $\mathrm{~F}$ & M & M & $\mathrm{U}$ \\
\hline 6 & $\mathrm{~F}$ & $\mathrm{U}$ & $\mathrm{U}$ & $\mathrm{F}$ \\
\hline 7 & M & M & $\mathrm{U}$ & $\mathrm{U}$ \\
\hline 8 & $\mathrm{~F}$ & $\mathrm{U}$ & $\mathrm{U}$ & $\mathrm{U}$ \\
\hline 9 & $\mathrm{M}$ & $\mathrm{U}$ & $\mathrm{F}$ & $\mathrm{U}$ \\
\hline 10 & $\mathrm{U}$ & M & $\mathrm{U}$ & $\mathrm{U}$ \\
\hline
\end{tabular}




\section{APPENDIX B}

Figure 6. Participant 1 Results

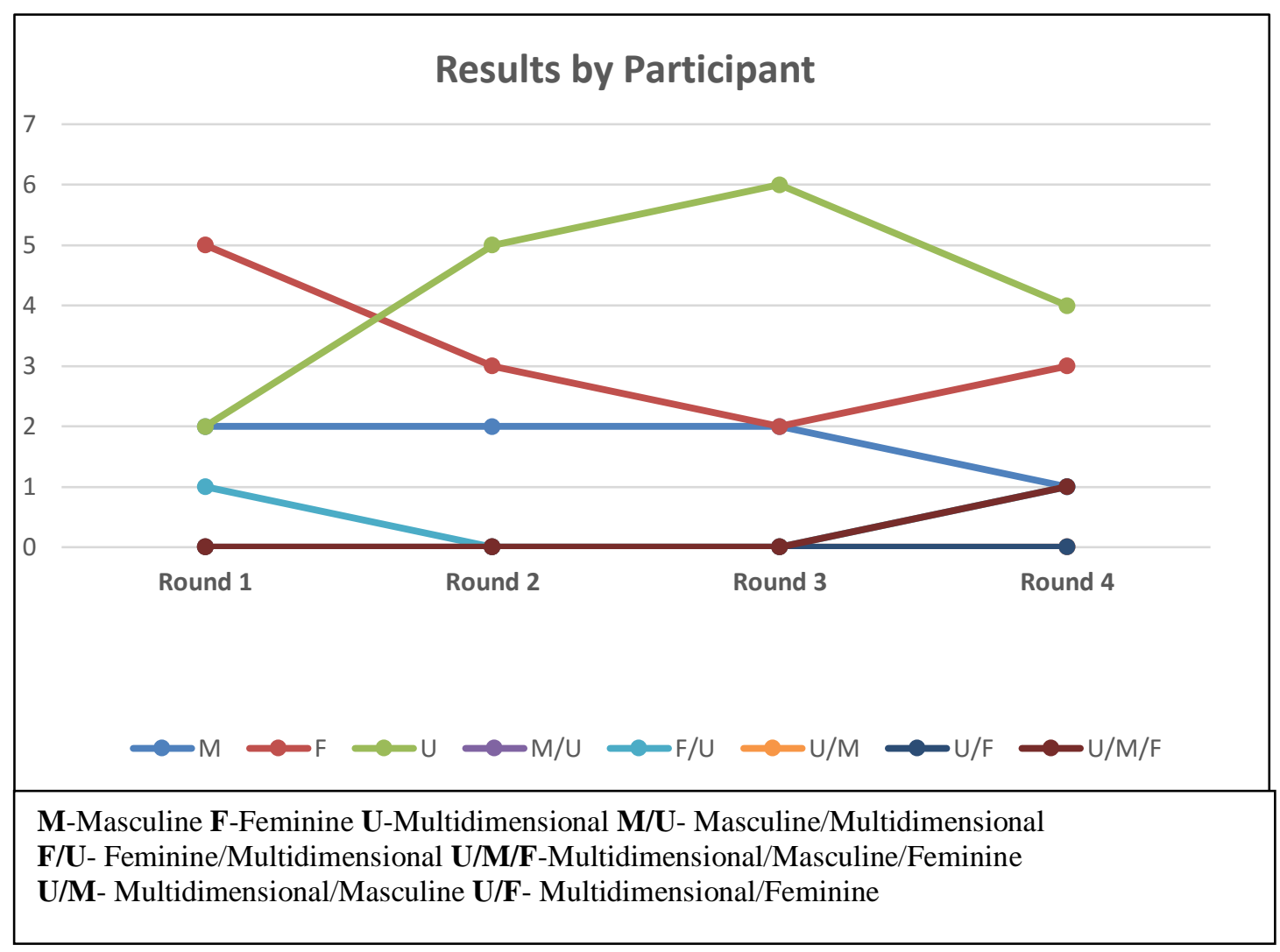


APPENDIX C

Figure 7. Participant 2 Results

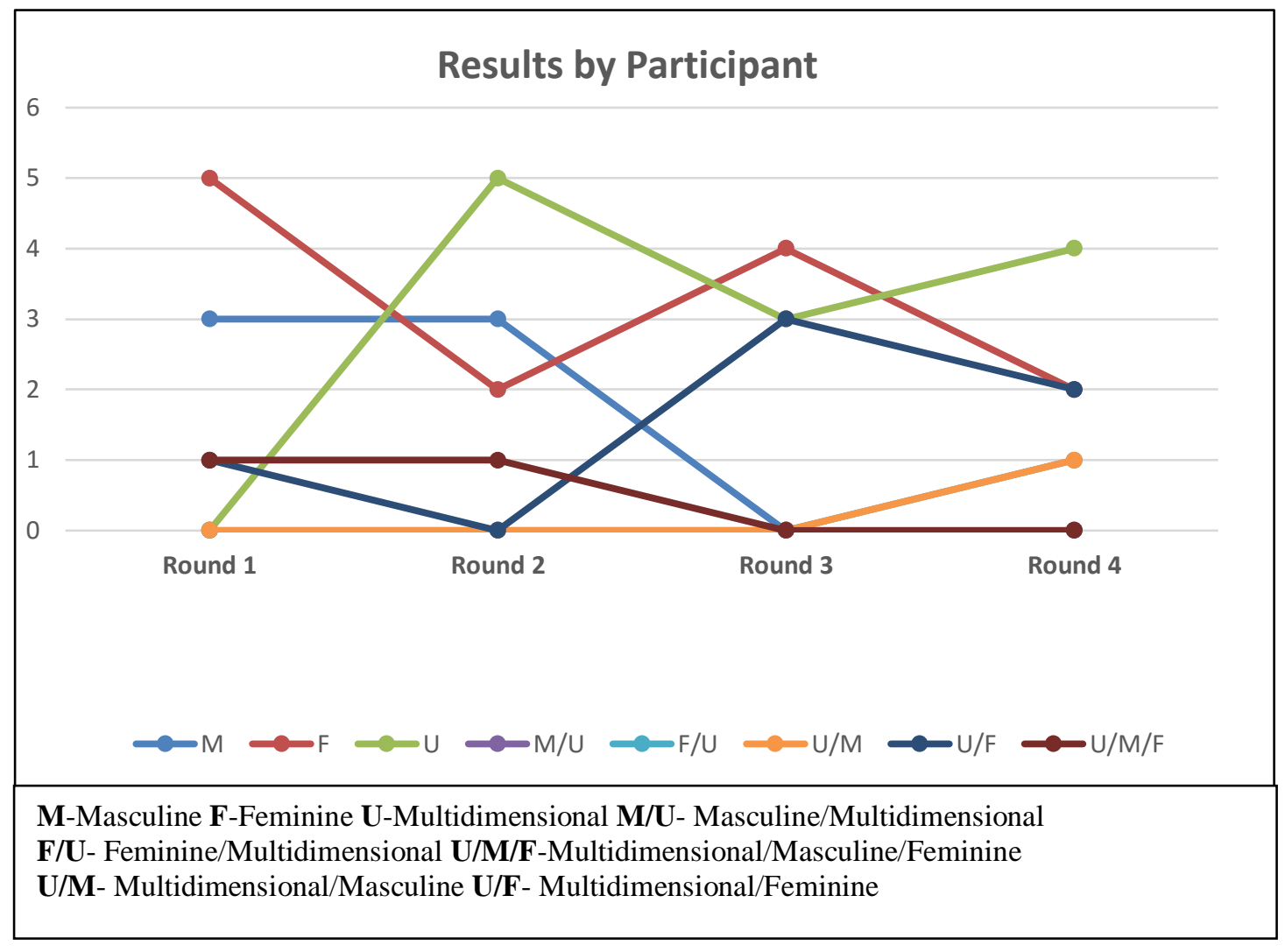




\section{APPENDIX D}

Figure 8. Participant 3 Results

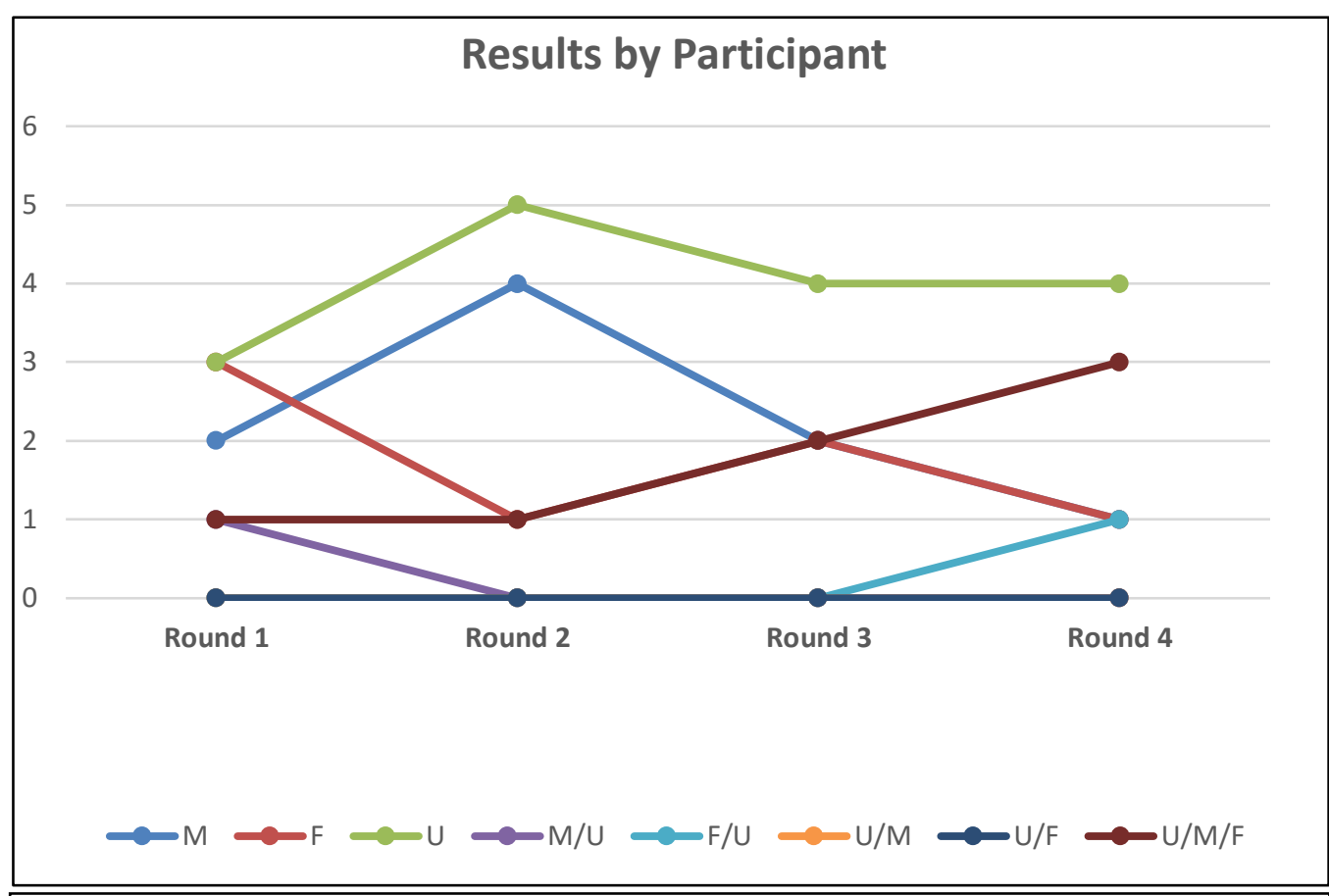

M-Masculine F-Feminine U-Multidimensional M/U- Masculine/Multidimensional

F/U- Feminine/Multidimensional U/M/F-Multidimensional/Masculine/Feminine

U/M- Multidimensional/Masculine U/F- Multidimensional/Feminine 


\section{APPENDIX E}

Figure 9. Participant 4 Results

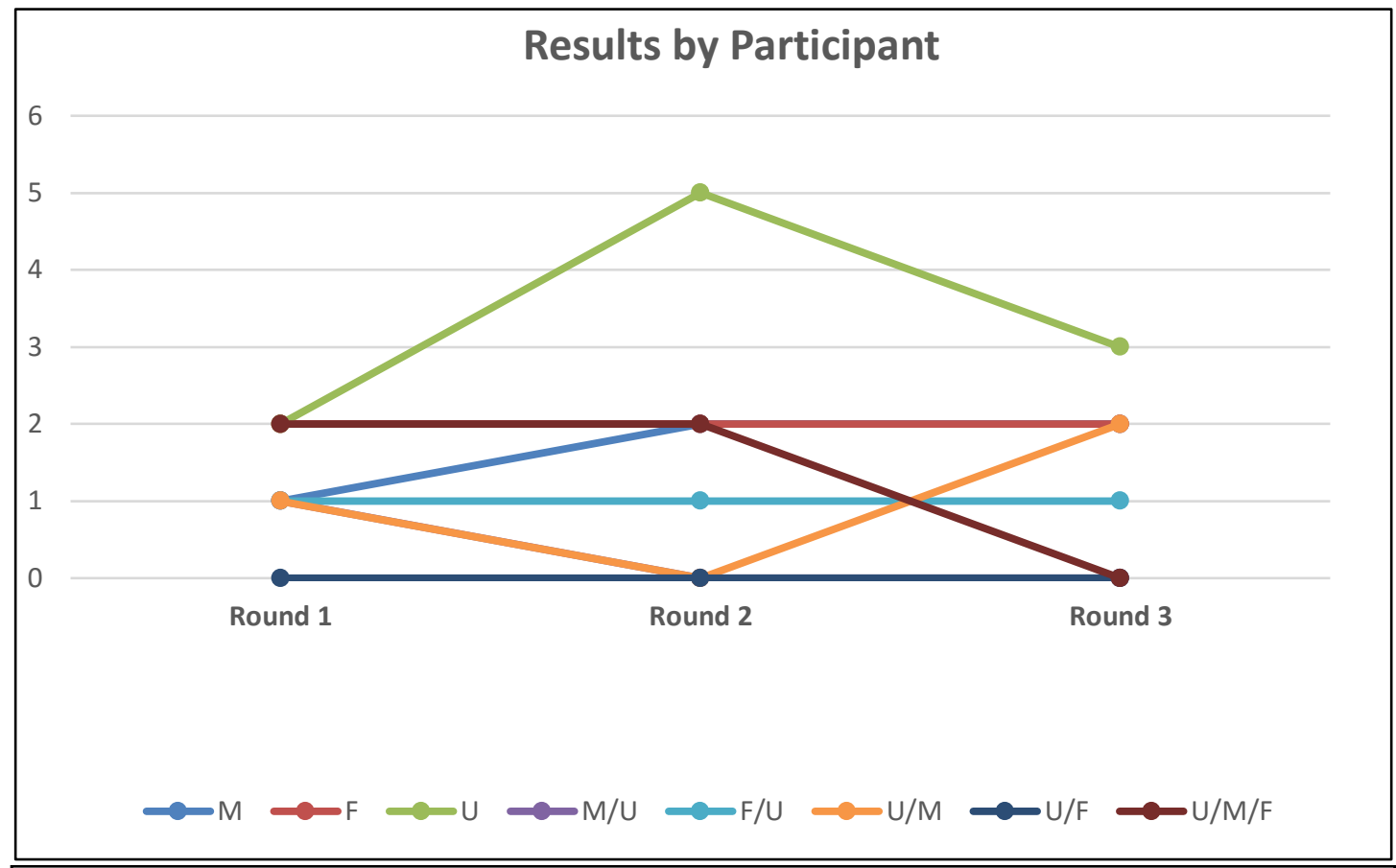

M-Masculine F-Feminine U-Multidimensional M/U- Masculine/Multidimensional

F/U- Feminine/Multidimensional U/M/F-Multidimensional/Masculine/Feminine

U/M- Multidimensional/Masculine U/F- Multidimensional/Feminine 


\section{APPENDIX F}

Figure 10. Participant 5 Results

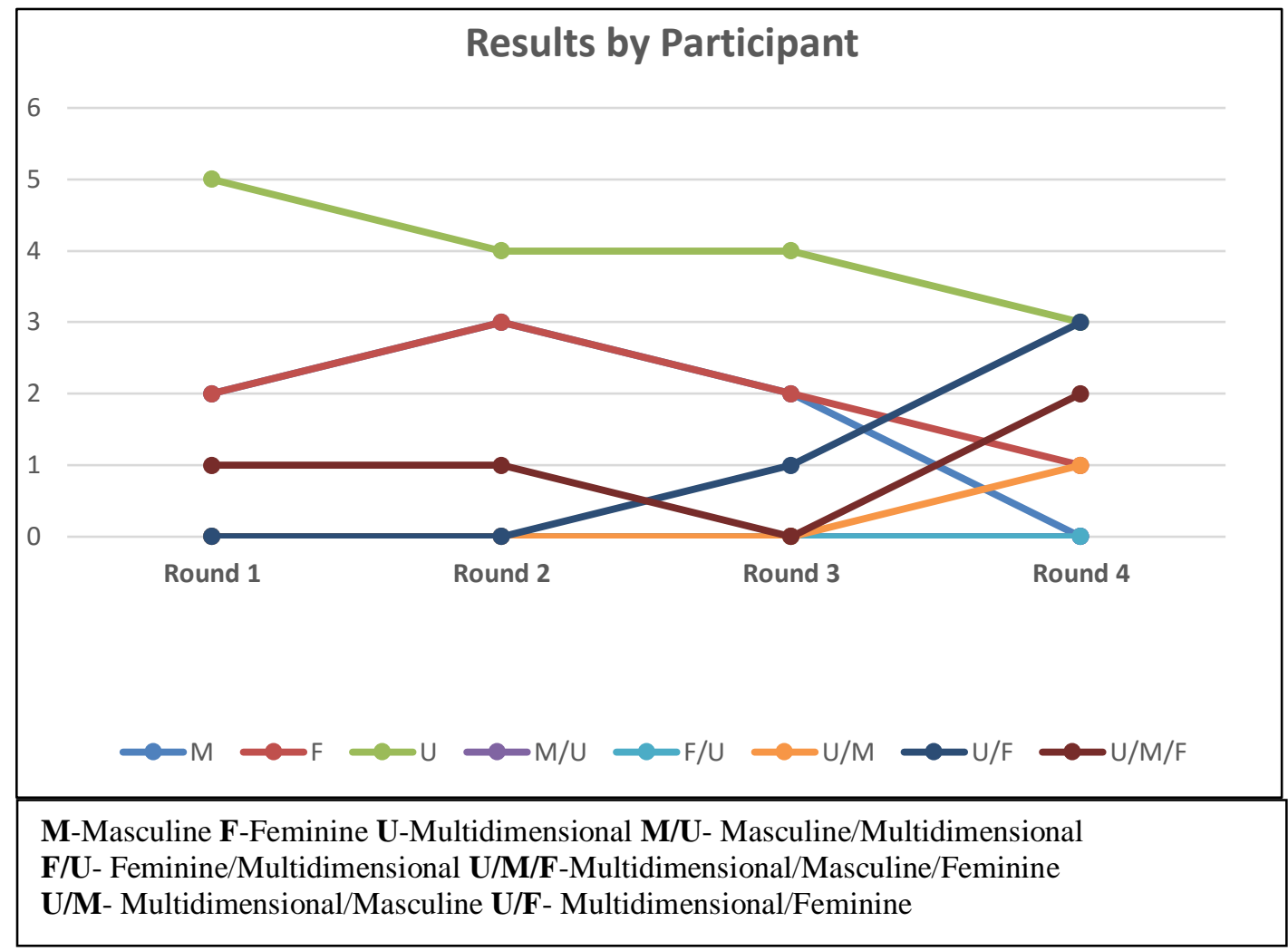




\section{APPENDIX G}

Figure 11. Participant 6 Results

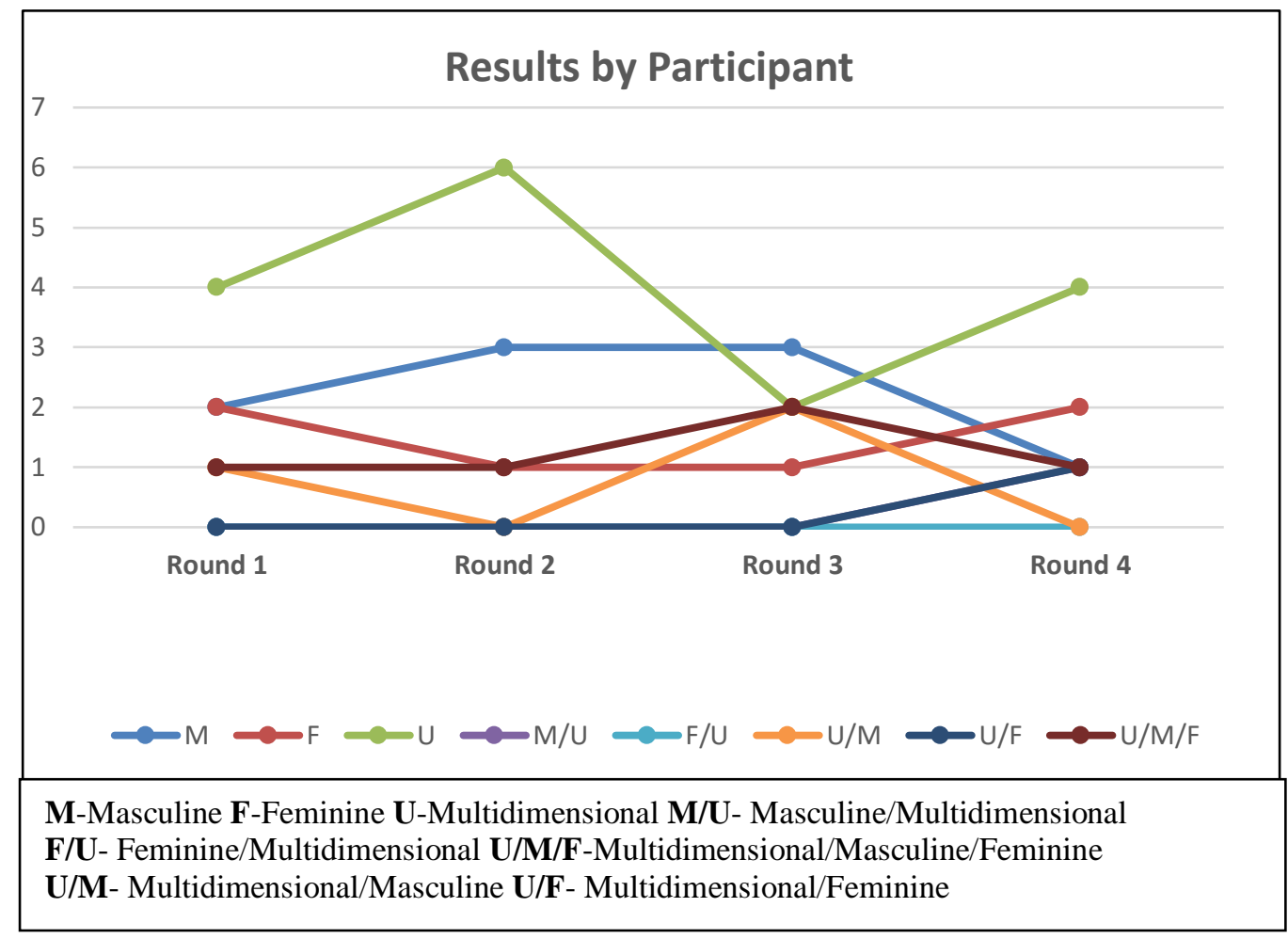




\section{APPENDIX H}

Figure 12. Participant 7 Results

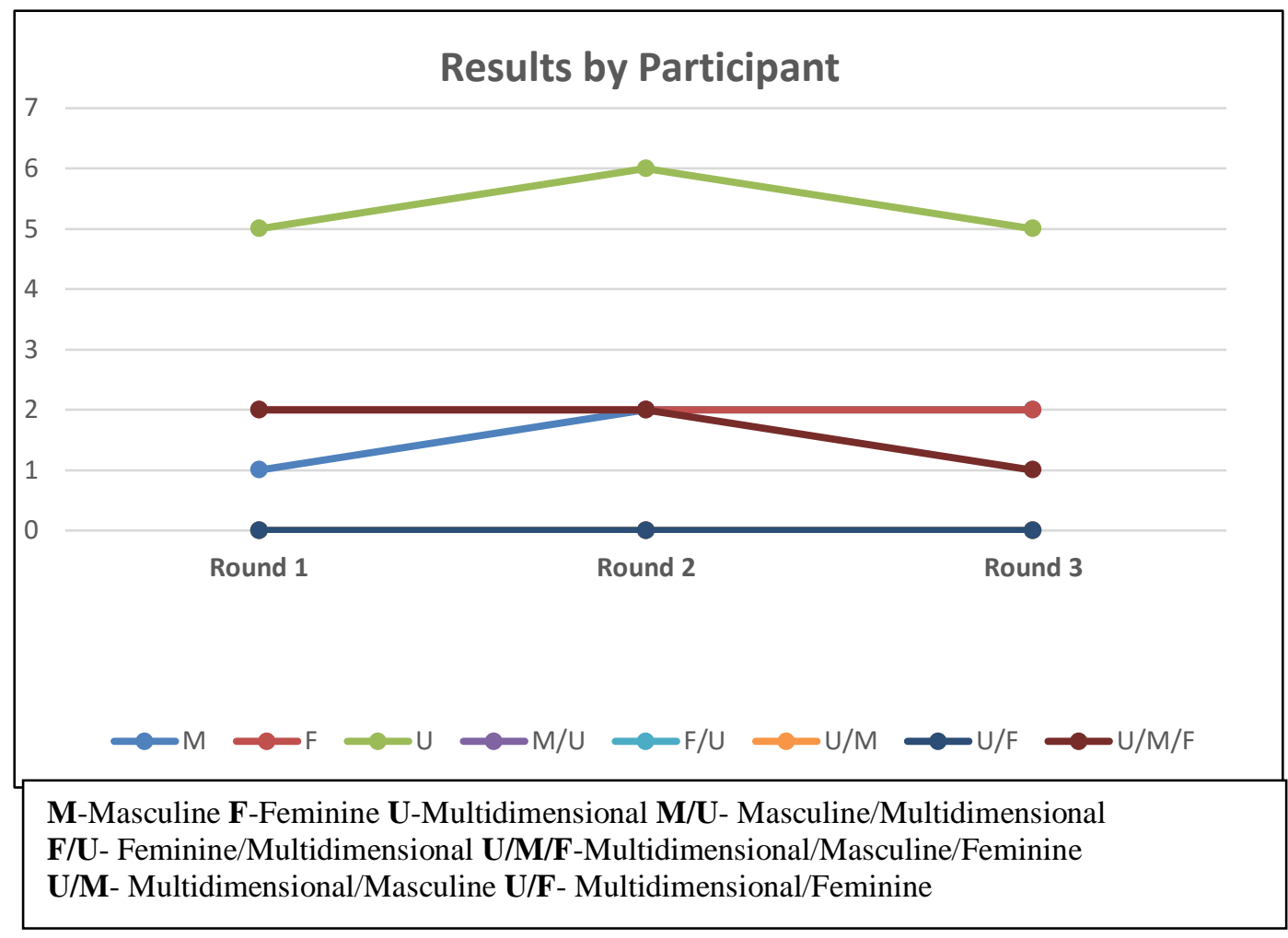




\section{APPENDIX I}

Figure 13. Participant 8 Results

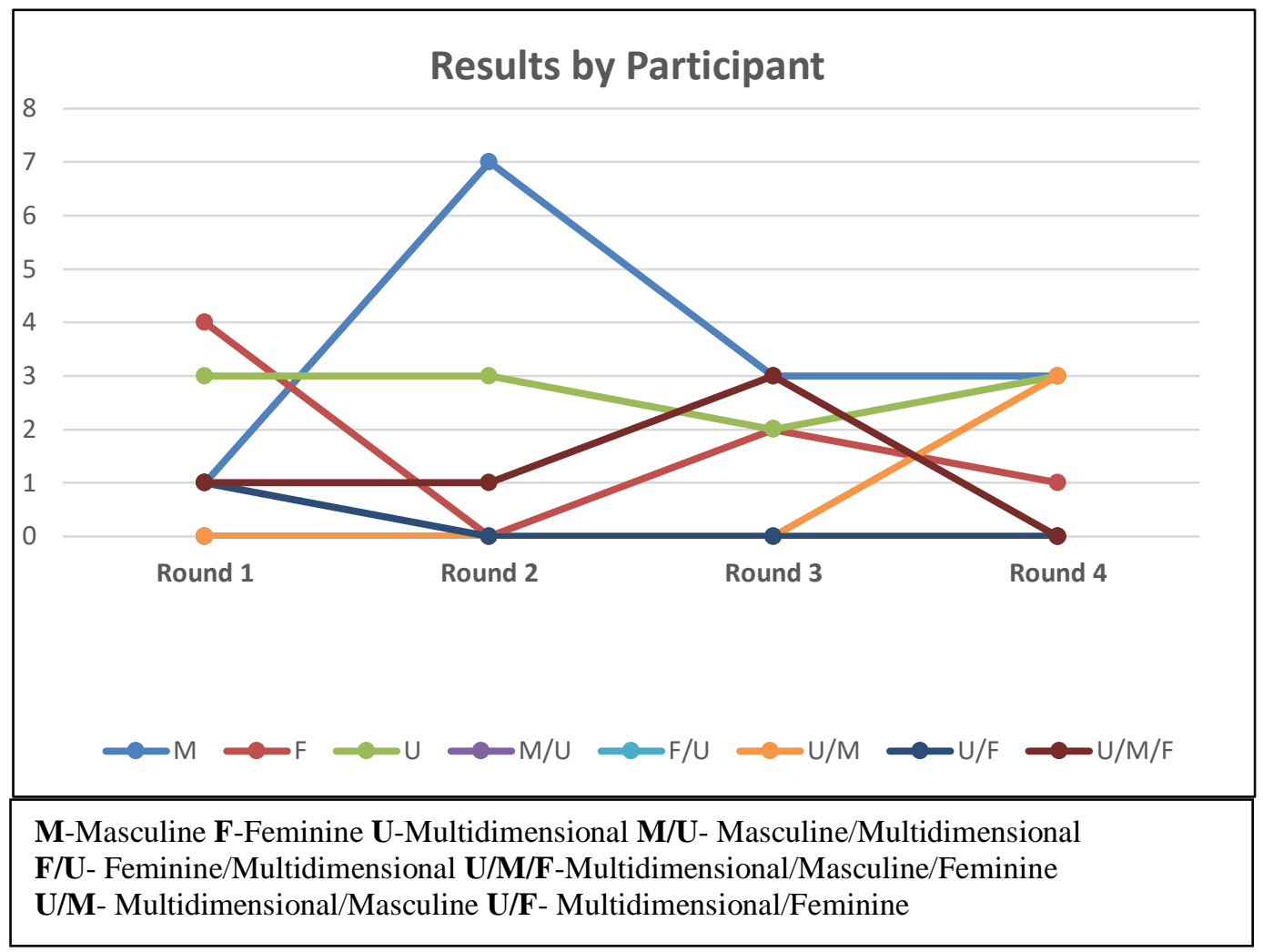




\section{APPENDIX J}

Figure 14. Participant 9 Results

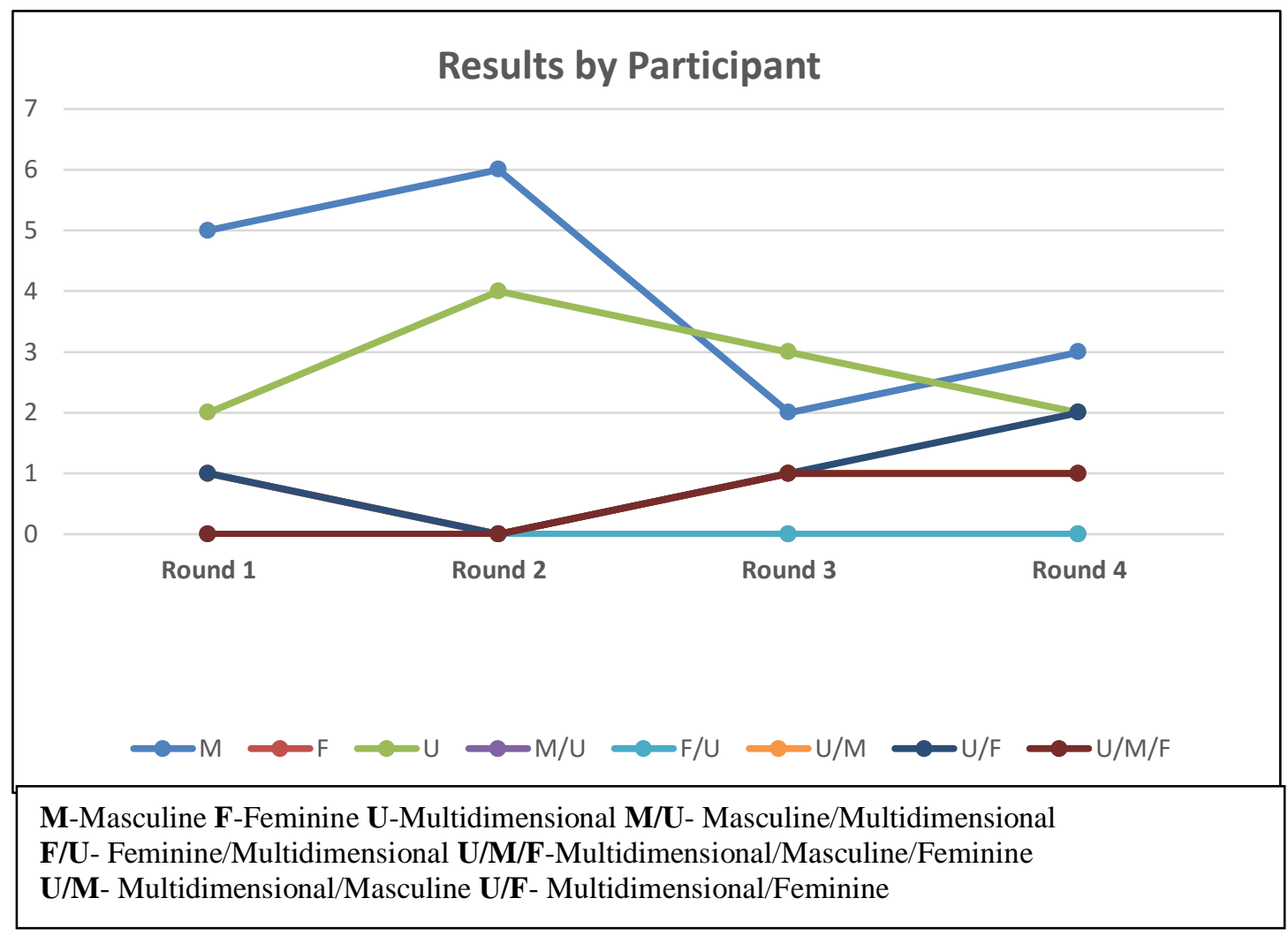




\section{APPENDIX K}

Figure 15. Participant 10 Results

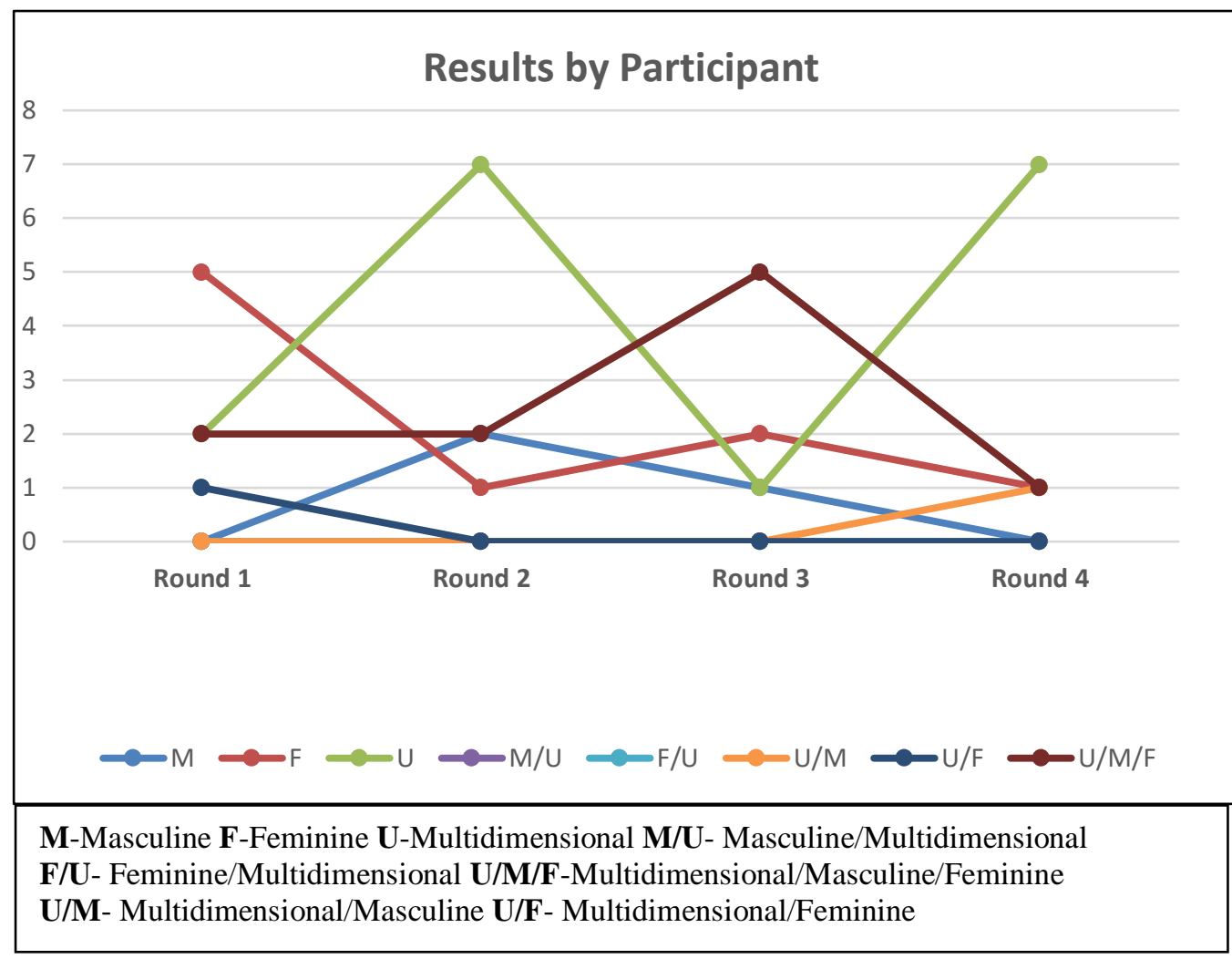




\section{APPENDIX L}

Figure 16. Participant 11 Results

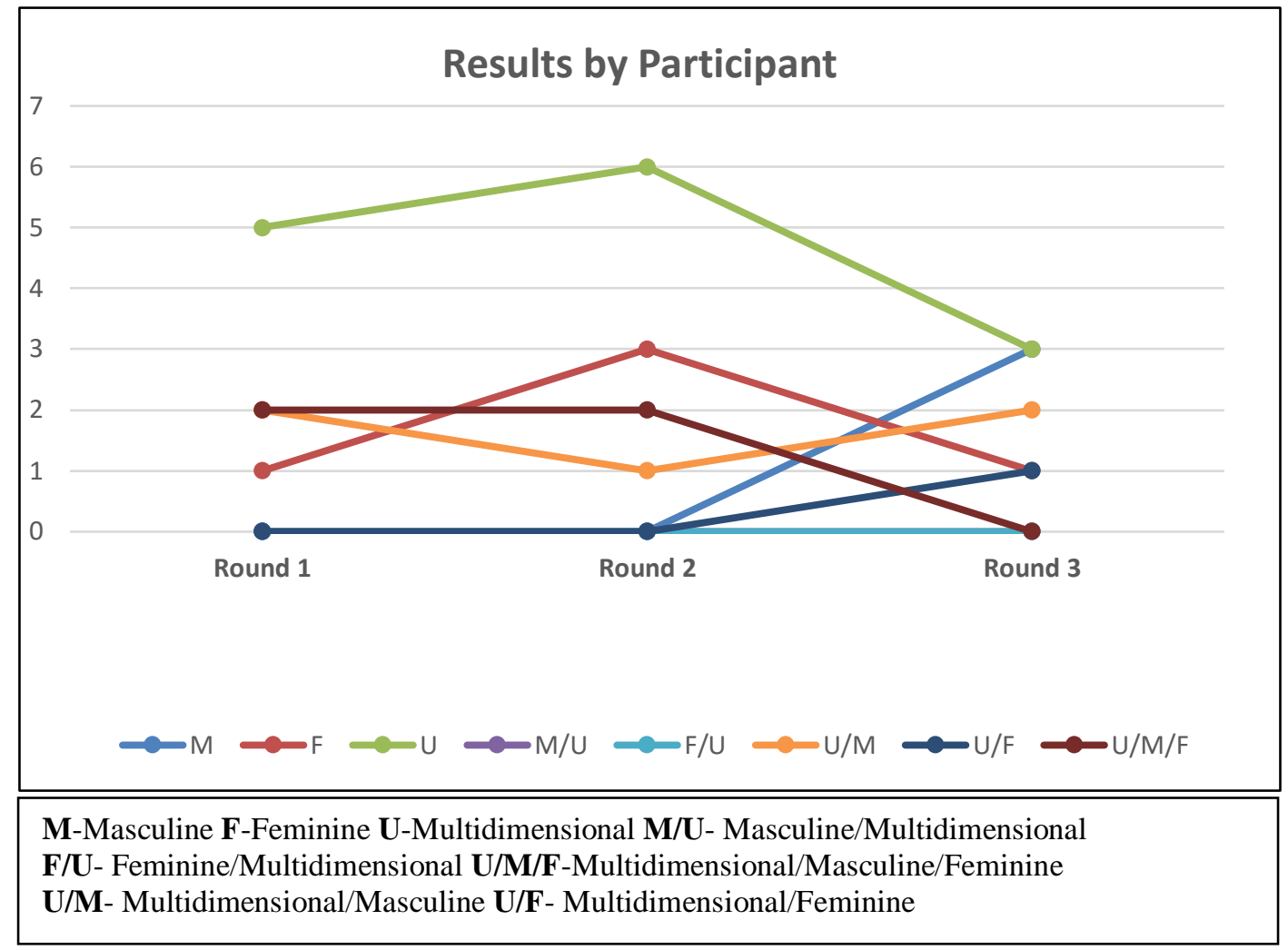




\section{APPENDIX M}

Figure 17. Participant 12 Results

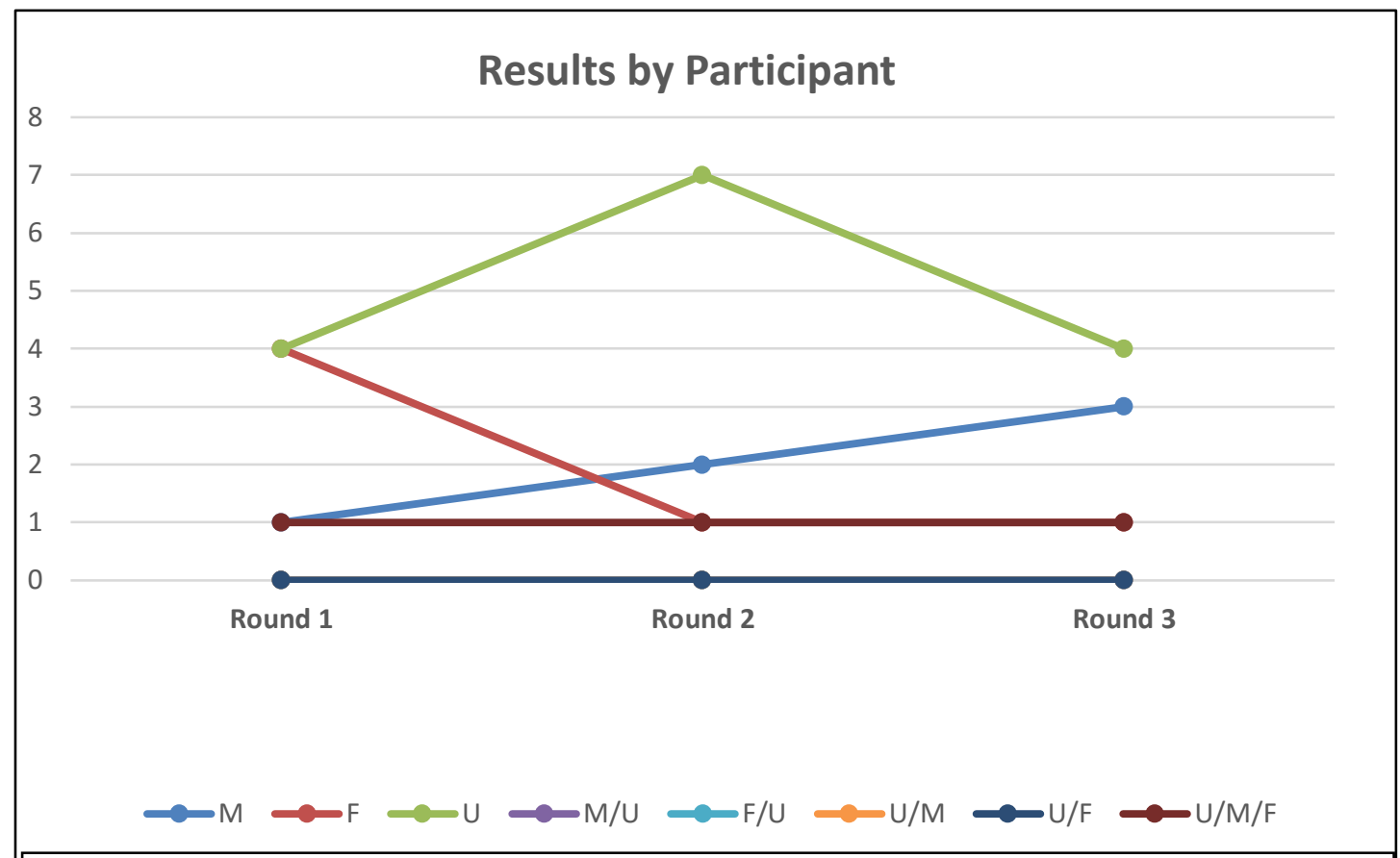

M-Masculine F-Feminine U-Multidimensional M/U- Masculine/Multidimensional F/U- Feminine/Multidimensional U/M/F-Multidimensional/Masculine/Feminine U/M- Multidimensional/Masculine U/F- Multidimensional/Feminine 


\section{APPENDIX N}

Figure 18. Participant 13 Results

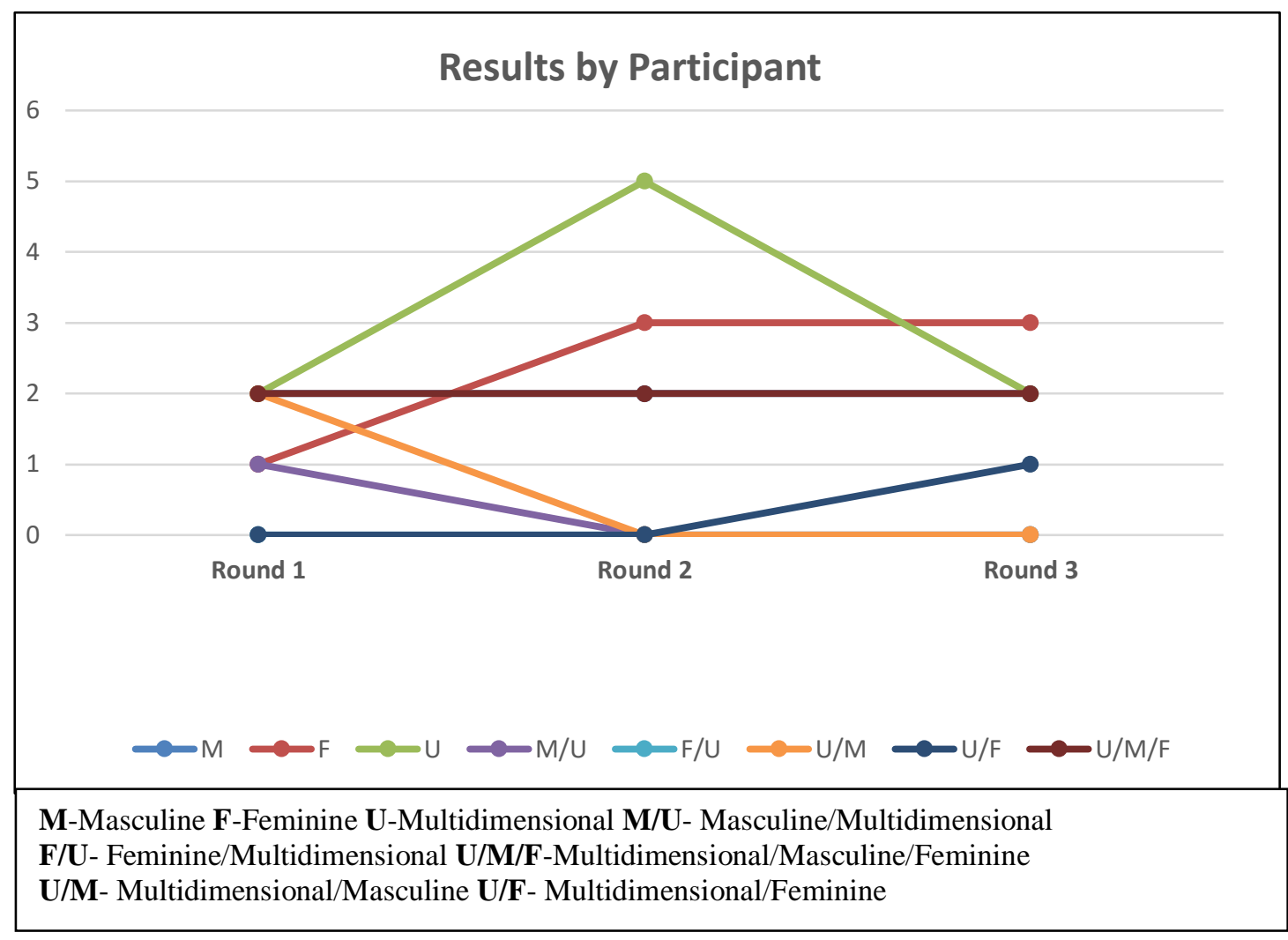




\section{APPENDIX O}

Figure 19. Participant 14 Results

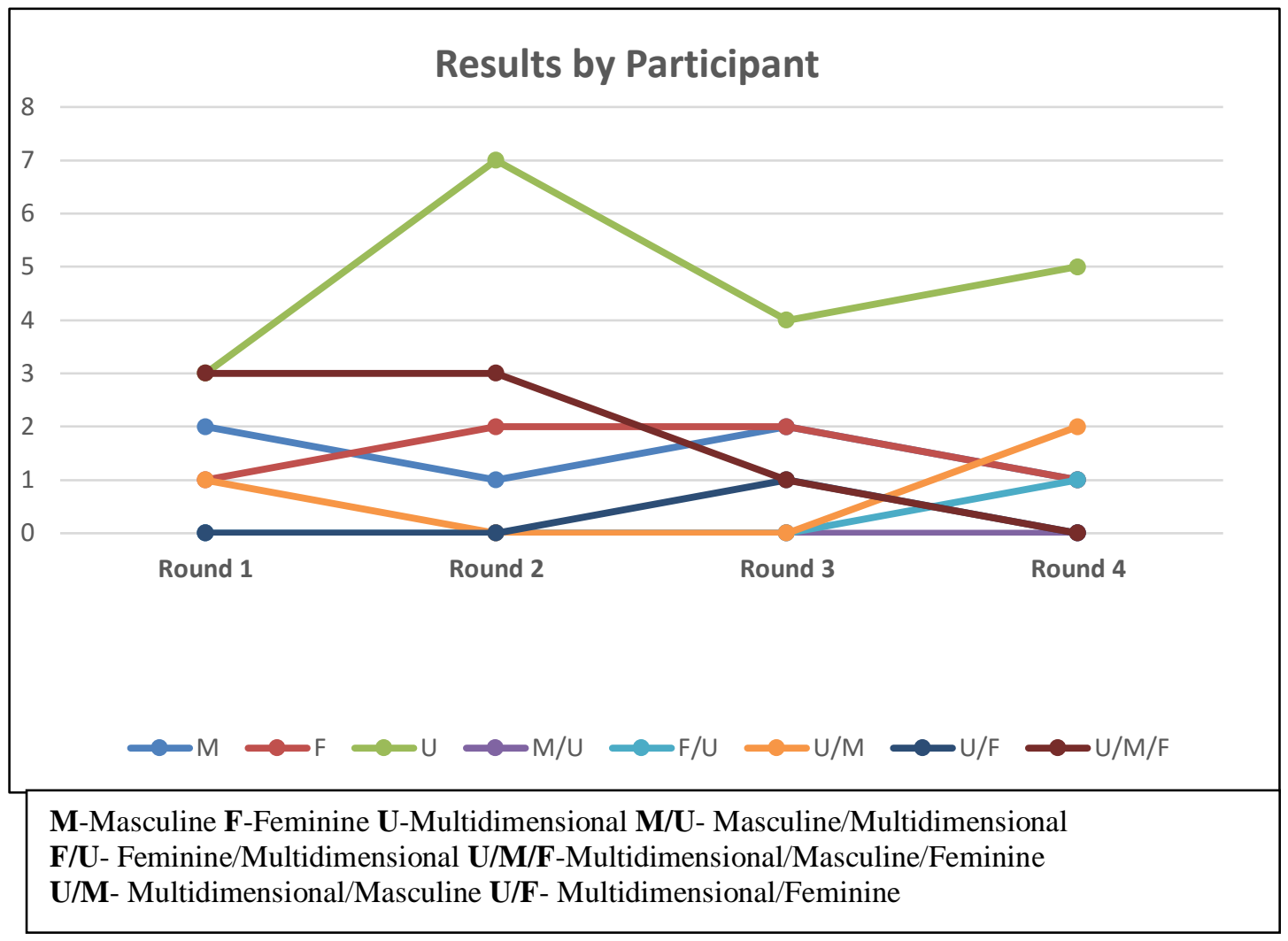




\section{APPENDIX P}

Figure 20. Participant 15 Results

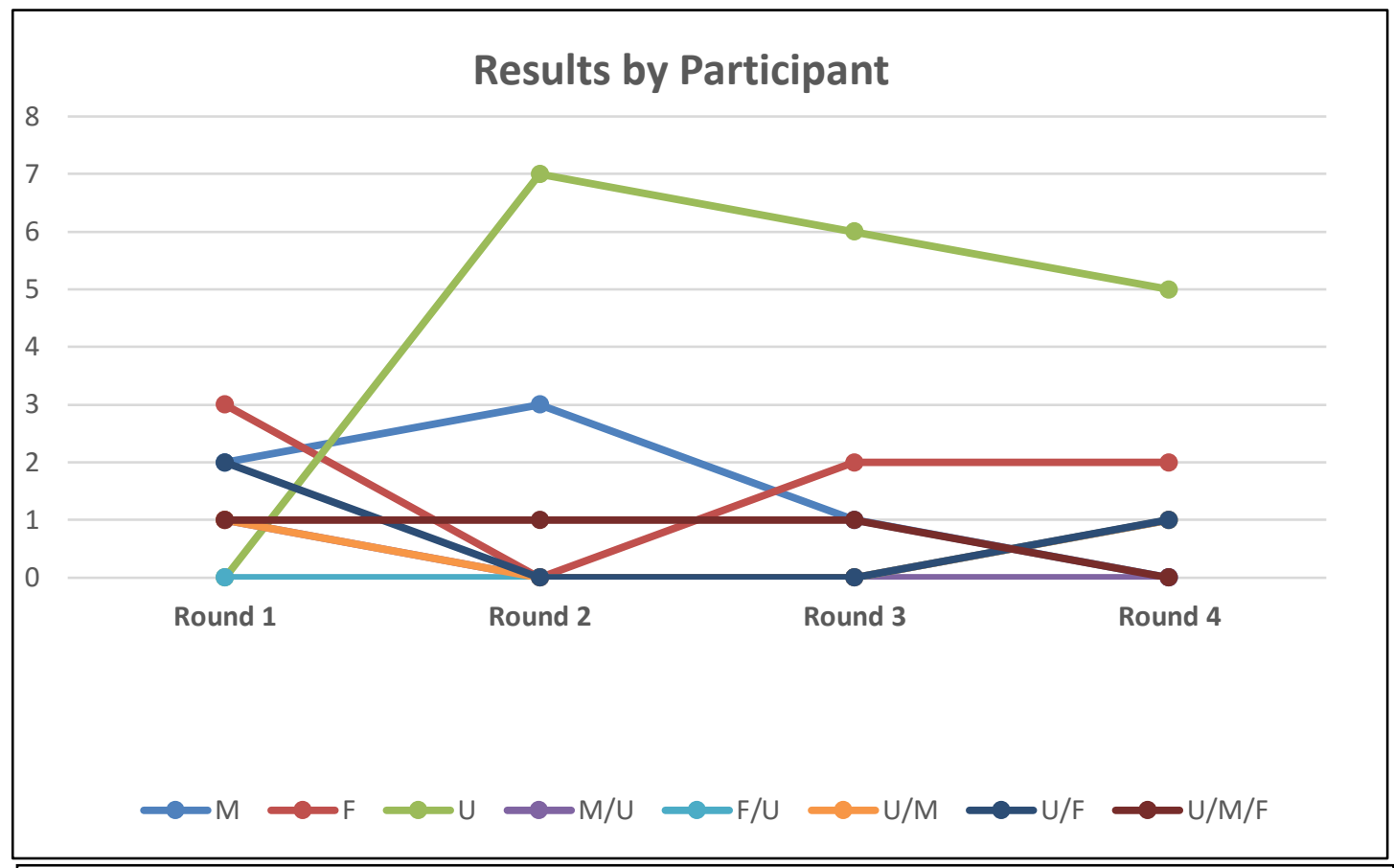

M-Masculine F-Feminine U-Multidimensional M/U- Masculine/Multidimensional

F/U- Feminine/Multidimensional U/M/F-Multidimensional/Masculine/Feminine

U/M- Multidimensional/Masculine U/F- Multidimensional/Feminine 


\section{APPENDIX Q}

Figure 21. Participant 16 Results

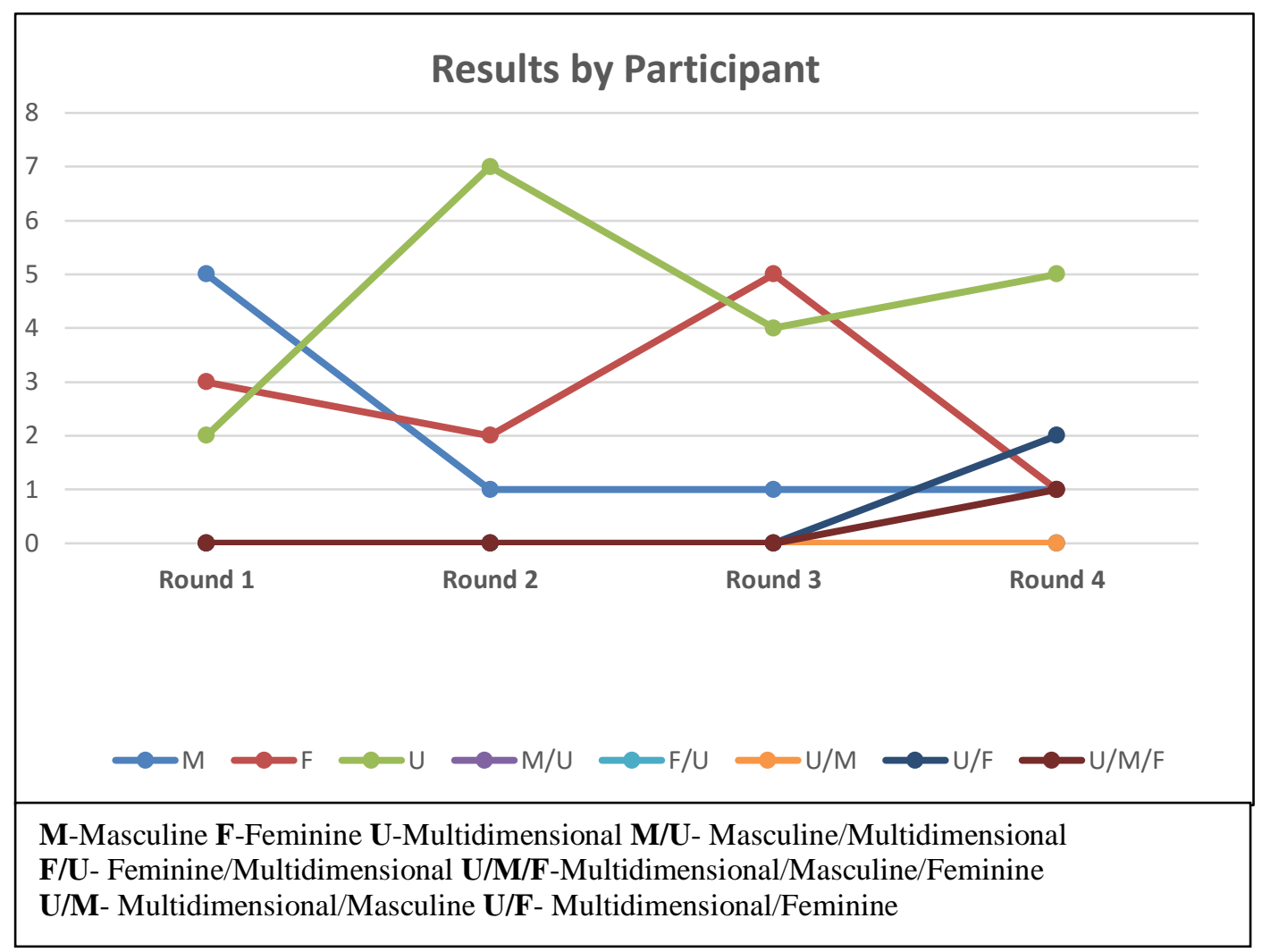




\section{APPENDIX R}

Figure 22. Participant 17 Results

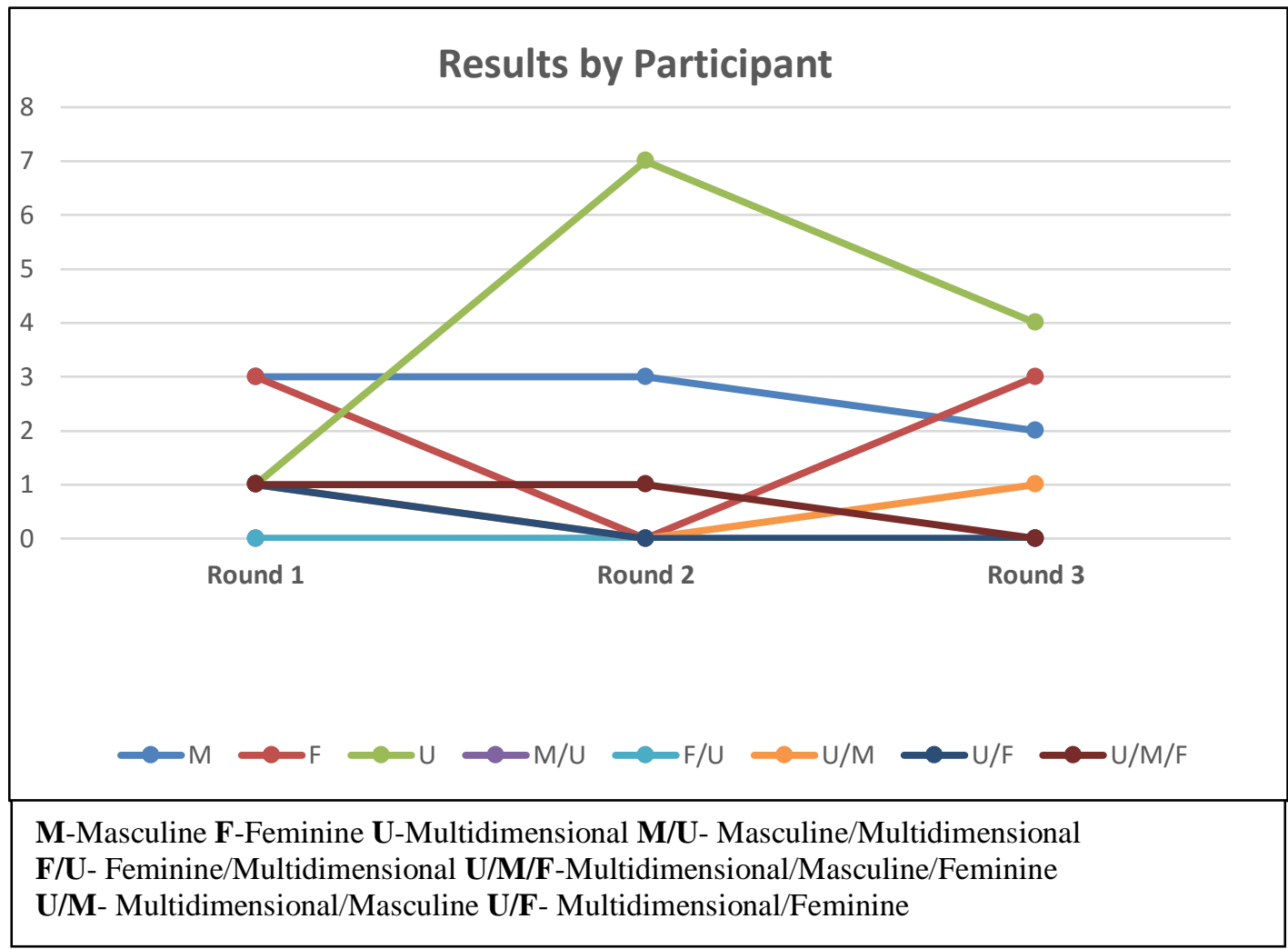




\section{APPENDIX S}

Figure 23. Participant 18 Results

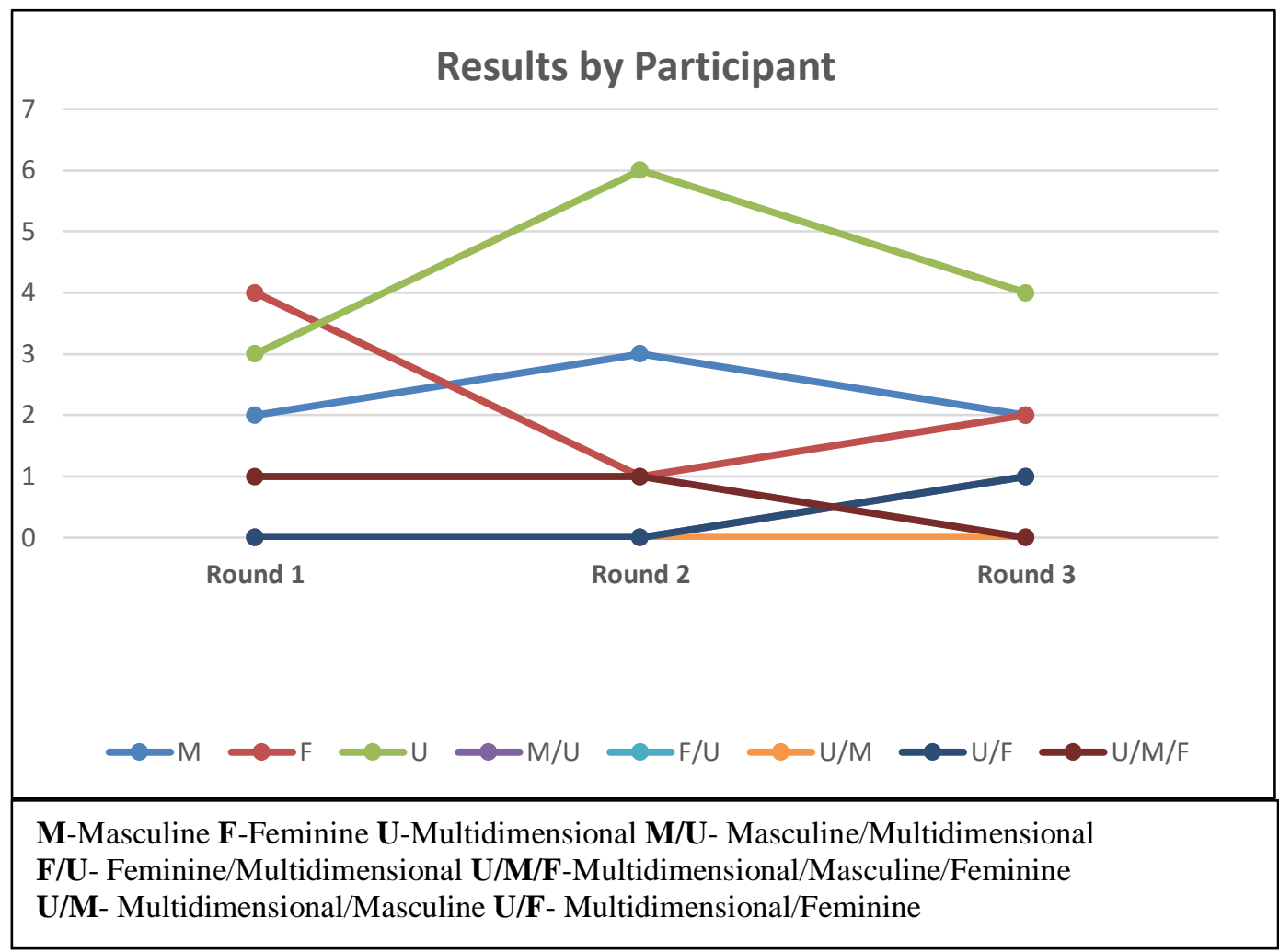




\section{APPENDIX T}

Figure 24. Participant 19 Results

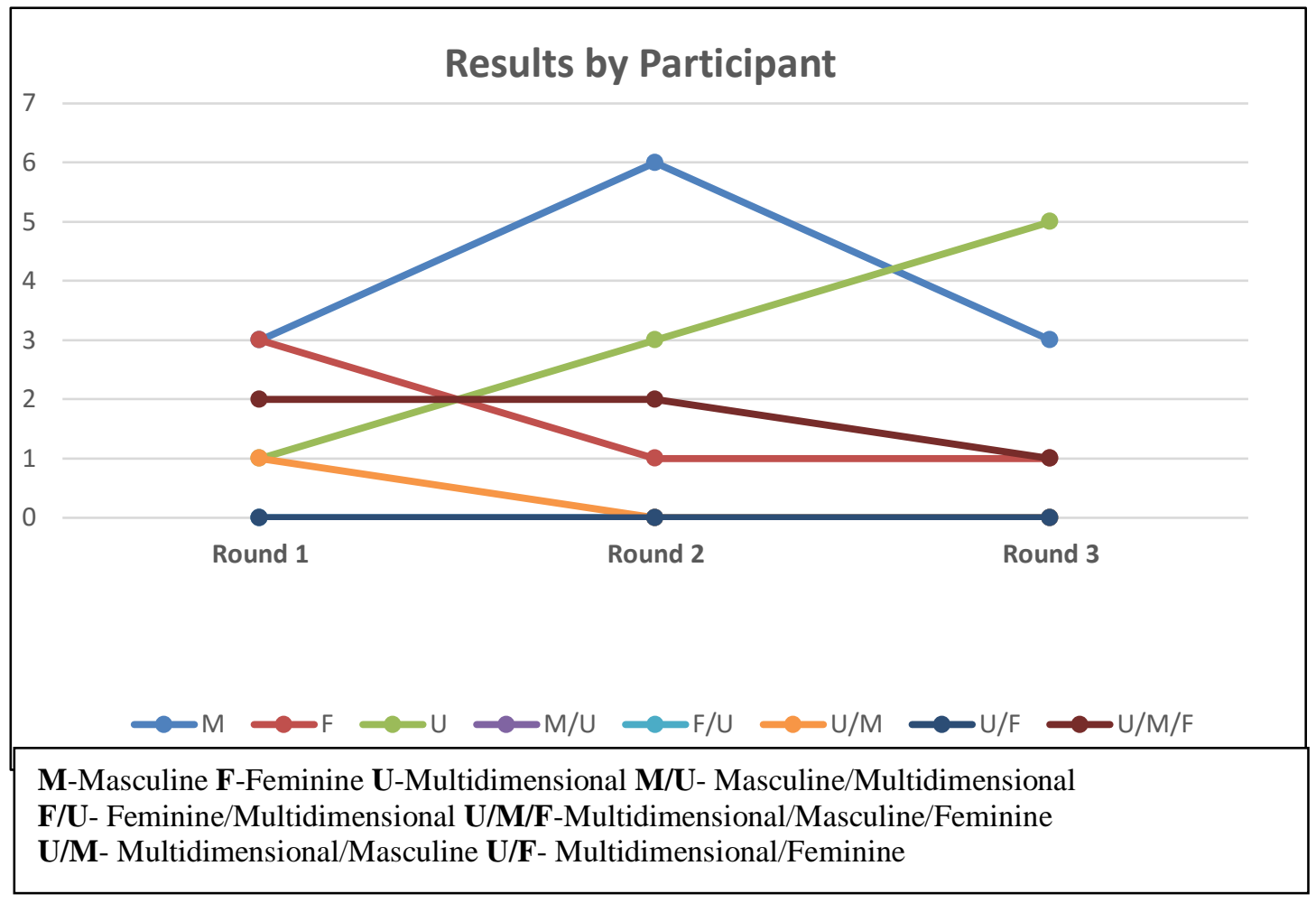




\section{APPENDIX U}

Figure 25. Participant 20 Results

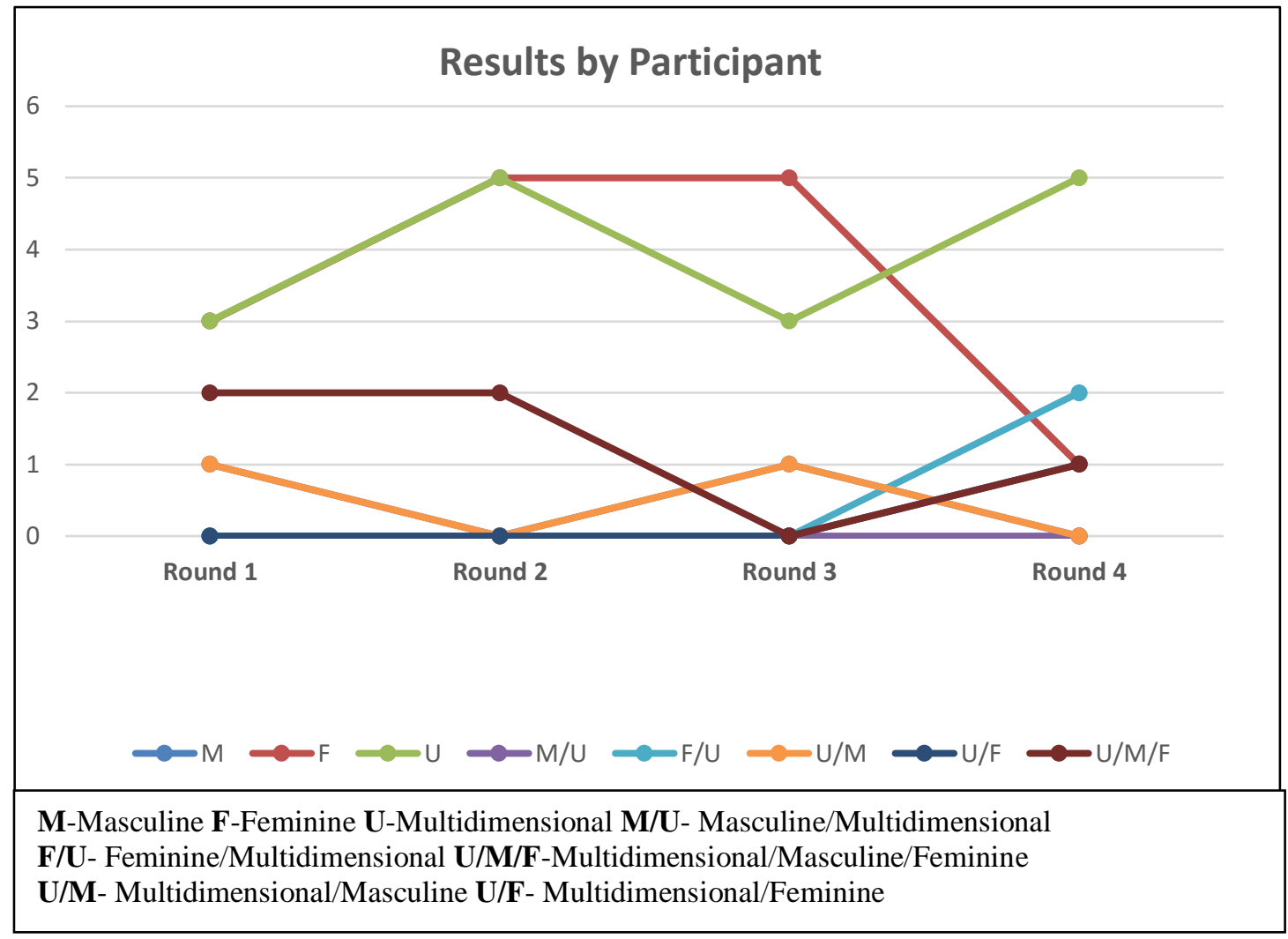




\section{APPENDIX V}

Table 4. Participant Demographic Information

\begin{tabular}{|c|c|c|c|c|c|}
\hline Participant & Position Title & $\begin{array}{l}\text { Years in } \\
\text { Position }\end{array}$ & $\begin{array}{l}\text { Years of } \\
\text { Service }\end{array}$ & Male & Female \\
\hline Participant \# 1 & $\begin{array}{l}\text { Curriculum Support } \\
\text { Specialist }\end{array}$ & 3 & 18 & & $\checkmark$ \\
\hline Participant \# 2 & $\begin{array}{l}\text { Reading Curriculum } \\
\text { Coach }\end{array}$ & 10 & 26 & & $\checkmark$ \\
\hline Participant \# 3 & $\begin{array}{l}\text { Community Liaison } \\
\text { Specialist }\end{array}$ & 12 & 12 & & $\checkmark$ \\
\hline Participant \# 4 & Administrative Director & 22.5 & 2.5 & & $\checkmark$ \\
\hline Participant \# 5 & Assistant Principal & 1 & 10 & & $\checkmark$ \\
\hline Participant \# 6 & Team Leader & 10 & 10 & & $\checkmark$ \\
\hline Participant \# 7 & Assistant Principal & 1 & 10 & & $\checkmark$ \\
\hline Participant \# 8 & Principal & 9 & 24 & & $\checkmark$ \\
\hline Participant \# 9 & $\begin{array}{l}\text { Motivational } \\
\text { Coach(Counselor) }\end{array}$ & 10 & 20 & $\checkmark$ & \\
\hline Participant \# 10 & Assistant Principal & 6 & 21 & & $\checkmark$ \\
\hline Participant \# 11 & Principal & 11 & 33 & & $\checkmark$ \\
\hline Participant \# 12 & $\begin{array}{l}\text { Reading Curriculum } \\
\text { Coach }\end{array}$ & 8 & 14 & & $\checkmark$ \\
\hline Participant \# 13 & Principal & $<1$ & 20 & & $\checkmark$ \\
\hline Participant \# 14 & Assistant Principal & 2 & 14 & & $\checkmark$ \\
\hline Participant \# 15 & Assistant Principal & .5 & 16 & & $\checkmark$ \\
\hline Participant \# 16 & Assistant Principal & 2 & 21 & & $\checkmark$ \\
\hline Participant \# 17 & Assistant Principal & 1 & 9 & $\checkmark$ & \\
\hline Participant \# 18 & $\begin{array}{l}\text { Curriculum Support } \\
\text { Specialist }\end{array}$ & .5 & 9 & $\checkmark$ & \\
\hline Participant \# 19 & Assistant Principal & 2 & 10 & $\checkmark$ & \\
\hline Participant \# 20 & $\begin{array}{l}\text { Science Curriculum } \\
\text { Coach }\end{array}$ & 4 & 27 & & $\checkmark$ \\
\hline
\end{tabular}




\section{APPENDIX W}

\section{Leadership Decision Making Questionnaire}

What is your position in the school in which you are employed?

How many years of experience do you have in your current position?

How many years of experience do you have in public education?

1. When seeking to foster a positive environment, explain how important it is to seek out the input from every individual in the building in maintaining a desired overall school culture: (F)

2. During the course of the school year, is it more important to be adaptable or stay loyal to the vision in which your stakeholders are committed? Explain your answer: (U/M/F)

3. How important is increasing shared involvement when both delegating and ensuring the completion of tasks? Please provide your reasoning: (U/M/F)

4. In your role as a leader, how has your journey and experience influenced your decision-making processes?

5. Explain how believing in yourself and others impacts your decision-making processes: (U/M/F)

6. Do you encourage your stakeholders to value family over work or do you expect them to keep priorities when it comes to work related responsibilities? Please explain your reasoning? (U)

7. Is succeeding the ultimate goal in endeavors pursued at your school and how do your decisions reflect obtaining set objectives? (M)

8. Explain how important your image is in reassuring stakeholders and colleagues of your leadership capacity?

9. As an educational leader, is it more important to be respected or loved? Please explain your answer: (U)

10. When taking on new responsibilities, explain the role that optimism has on the achievement of goals: (U) 


\section{APPENDIX X}

\section{Situational Decision Making Survey}

Please read through each short scenario and select the answer choice that best reflects the decision you would most likely choose.

1 A directive has been sent straight from the school board to be implemented within the school district, you:

a) independently study the request and come up with a game plan to present to your school/department. (M)

b) quickly gather a leadership team to disseminate responsibility. (F)

c) devise a plan and further develop the appropriate steps with colleagues to implement an achievable plan. (U)

d) Other. Please explain an alternative decision.

2. You have determined that the current action plan put into place at your school/department is falling short of expectations, you:

a) survey your leadership team to identify possible steps for improvement and utilize the input from stakeholders to develop a plan to reconstitute your efforts. (U)

b) accept responsibility for the lack of improvement and devise a plan to accommodate the needs of your school/department. (M)

c) work with individuals in a trusted group of colleagues and brainstorm how to redirect your efforts. (F)

d) Other. Please explain an alternative decision.

3. The pile of responsibilities on your desk never seems to decrease, you:

a) take time to carefully delegate tasks as to share in the weight of circumstances. $(\mathrm{F})$

b) identify a strategic plan to take care of tasks with greater consequences and work with your leadership team to delegate duties. (U)

c) recognize that greater responsibility comes to individuals in your position causing you to reinvest your commitment to working more efficiently. (M)

d) Other. Please explain an alternative decision.

4. You are being asked to make a significant decision that impact your school/department, you:

a) ask a trusted group of individuals to collaborate on a decision that will be more beneficial to your mission. (F)

b) rely on your experience to study the consequences of each possible action and devise how to best go about making the proper choices. (M)

c) highlight key points in possible choices and share these potential decisions with others to seek their input. (U)

d) Other. Please explain an alternative decision.

5. The enthusiasm that once existed due to taking on a new job has waned and it appears that you have not been able to perform according to the expectations that were set by yourself and others, you: a) reflect on the optimism that you have for your work ethic and devise a plan that will best serve the purpose that you originally set out to achieve. (M)

b) share with trusted stakeholders your belief in each other and devise a reconstitution of efforts to ensure success. (U)

c) ask others to contribute to creating a culture that will increase optimism towards the vision and mission of your school/department. (F)

d) Other. Please explain an alternative decision. 
6. It is December and an individual that you supervise has recorded 10 absences from work this school year. This individual has just called and requested for three personal days. He/She has been open about family turmoil, you:

a) work with the appropriate support services to help advise your colleague on possible options moving forward and to personally show support for him/her. (U)

b) recognize that the individual is experiencing turmoil at home and emphasize the importance of family and managing a healthy balance in life. $(\mathrm{F})$

c) meet with the individual to reinstate the vision and mission of your organization and share how important they are to that cause. (M)

d) Other. Please explain an alternative decision.

7. While closing the door to your office you are reminded about your commitments to raising student achievement. State assessment scores from the previous school-year have just been published and they were lower than expected, you:

a) reflect on your experience, devise a plan to improve student performance, and put actions into place that will serve your overall mission. (M)

b) call individual stakeholders to a meeting to collaborate on an appropriate response. (F)

c) both recognize the importance of state assessments and that the success of your school/department is also measured by other statistics that were not mentioned in the publication.

(U)

d) Other. Please explain an alternative decision.

8. You are preparing a statement to make to colleagues and over 300 community stakeholders, you:

a) carefully write your announcement and share it with a trusted group of individuals to receive their input. (U)

b) ask individuals that are a part of your team to collaborate on an appropriate statement. (F)

c) highlight key points that are significant to you and that will ensure the delivery of a clear and exact message. (M)

d) Other. Please explain an alternative decision.

9. Walking up and down the halls of your school/department you have heard sharp criticisms of your approach to increase student achievement, you:

a) understand that criticism comes with the territory, which should not be taken personally. (M)

b) genuinely ask for the input of stakeholders to share possible areas for improvement and incorporate their responses into decisions going forward. (U)

c) recognize that trust is an important part of a team approach, which prompts a change in your approach. (F)

d) Other. Please explain an alternative decision.

10. You have just received a promotion and despite feeling honored by the increase in salary and responsibility, the challenges seem to be overwhelming, you:

a) believe in yourself and the potential of your colleagues to share in new positive effort to overcome perceived obstacles. (M)

b) know challenges are expected and reinstate the belief that working hard and sharing a positive attitude will help you in overcoming these challenges. (U)

c) work with your new colleagues to develop a positive and meaningful approach to overcoming obstacles. (F)

d)Other. Please explain an alternative decision. 


\section{Appendix Y}

\section{Round Three Survey Reflections}

*This round has been provided to reflect, respond and/or revise the decisions chosen in the Situational Decision Making Survey. Please provide as much detail as necessary to convey your decision-making thought processes.

Please revise and/or reflect on the information provided for each item below:

1. A directive has been sent straight from the school board to be implemented within the school district, you:
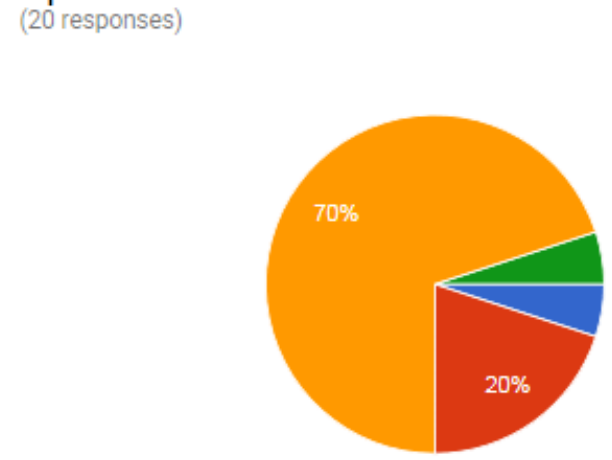

independently study the request and come up with a game plan to present to your school/department.

- quickly gather a leadership team to disseminate responsibility

devise a plan and further develop the appropriate steps with colleagues to implement an achievable plan.

Other

2. You have determined that the current action plan put into place at your school/department is falling short of expectations, you:

(20 responses)

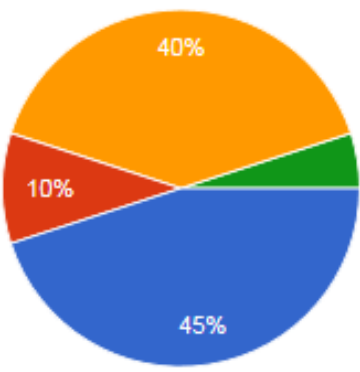

survey your leadership team to identify possible steps for improvement and utilize the input fr. accept responsibility for the lack of improvement and devise a plan to accommodate the needs of your sc.

work with individuals in a trusted group of colleagues and brainstorm how to redirect your efforts.

Other 
3. The pile of responsibilities on your desk never seems to decrease, you: (20 responses)

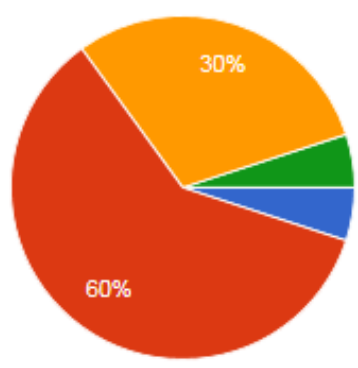

take time to carefully delegate tasks as to share in the weight of

circumstances.

identify a strategic plan to take care of tasks with greater consequences and work with your leadership team to $d$..

recognize that greater responsibility comes to individuals in your position causing you to reinvest your commit..

Other

4. You are being asked to make a significant decision that will impact your school/department, you:

(20 responses)

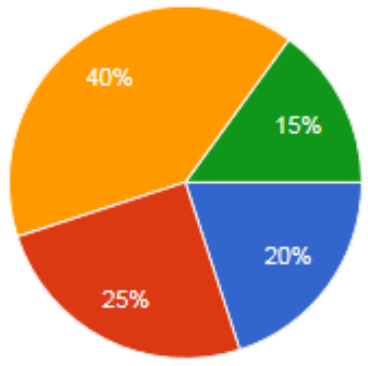

ask a trusted group of individuals to collaborate on a decision that will be more beneficial to your mission.

rely on your experience to study the consequences of each possible action and devise how to best go about $\mathrm{m}$...

highlight key points in possible choices and share these potential decisions with others to seek their i...

Other

5. The enthusiasm that once existed due to taking on a new job has waned and it appears that you have not been able to perform up to the expectations that were set by yourself and others, you:

(20 responses)

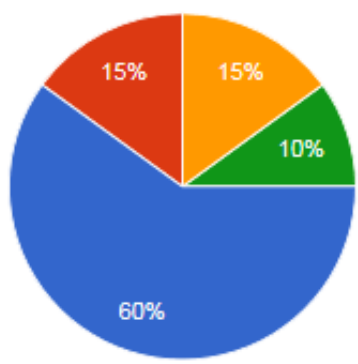

reflect on the optimism that you have for your work ethic and devise a plan that will best serve the purpose that.

share with trusted stakeholders your belief in each other and devise a reconstitution of efforts to ensure su...

ask others to contribute to creating a culture that will increase optimism towards the vision and mission of $y$...

Other 
6. It is December and an individual that you supervise has recorded 10 absences from work this school-year. This individual has just called and requested for three personal days. He/She has been open about family turmoil, you:

(20 responses)

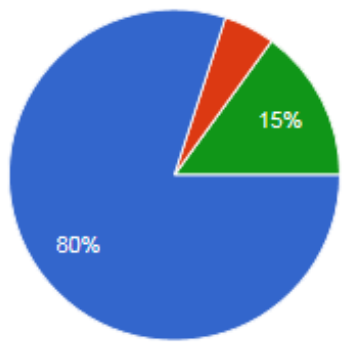

work with the appropriate support services to help advise your colleague on possible options moving forward..

recognize that the individual is experiencing turmoil at home and emphasize the importance of family..

meet with the individual to reinstate the vision and mission of your organization and share how importa.

Other

7. While closing the door to your office, you are reminded about your commitments to raising student achievement. State assessment scores from the previous school-year have just been published and they were lower than expected, you:

(20 responses)

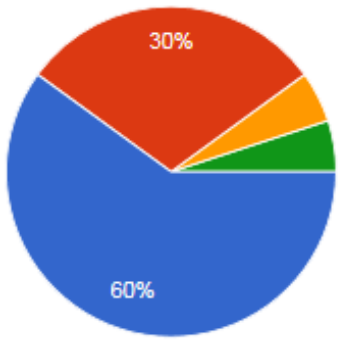

reflect on your experience, devise plan to improve student performance, and put actions into place that will s..

call individual stakeholders to a meeting to collaborate on an appropriate response.

both recognize the importance of state assessments and that the success of your school/department i...

Other

8. You are preparing a statement to make to colleagues and over 300 community stakeholders, you:

(20 responses)

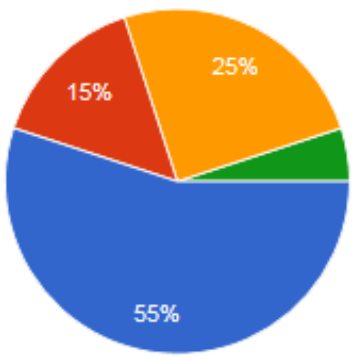

carefully write your announcement and share it with a trusted group of individuals to receive their input.

ask individuals that are a part of your team to collaborate on an appropriate statement.

highlight key points that are significant to you and that will ensure the delivery of a clear and exact messa...

Other 
9. Walking up and down the halls of your school/department, you have heard sharp criticisms of your approach to increase student achievement, you:

(20 responses)

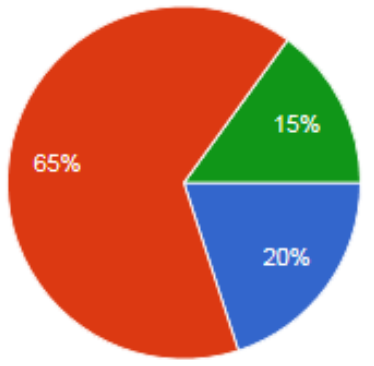

understand that criticism comes with the territory, which should not be taken personally.

genuinely ask for the input of stakeholders to share possible areas for improvement and incorporate th...

recognize that trust is an important part of a team approach, which prompts a change in your approach

Other

10. You just received a promotion and despite feeling honored by the increase in salary and responsibility, the challenges seem to be overwhelming, you:

(20 responses)

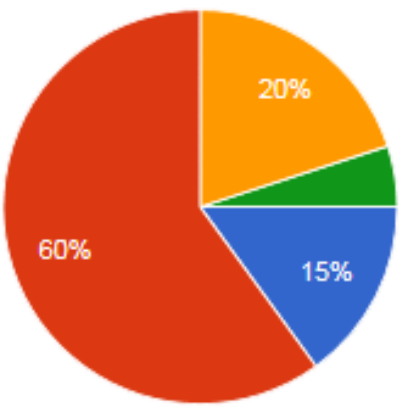

believe in yourself and the potential of your colleagues to share in new positive effort to overcome perceive...

know challenges are expected and reinstate the belief that working hard and sharing a positive attitude will $\mathrm{h}$.

work with your new colleagues to develop a positive and meaningful approach to overcoming obstacles.

Other 


\section{Appendix Z}

\section{Round Four: Final Reflections}

This round serves as an opportunity for all participants to take a glimpse of the responses from their colleagues and compare them with both the consensus developed during the course of the study as well as with his/her personal reflections. Although the input of each participant is valued for each item, participation in this round is not required, but rather requested.

Please look at each item and read the PARTICIPANT INPUT details provided. Offer your final reflections on what leadership decision making steps may be missing.

\section{A directive has been sent straight from the school board to be implemented within the school district, you:}

* $73.7 \%$ of participants chose: devise a plan and further develop the appropriate steps with colleagues to implement an achievable plan.

\section{Participant Input:}

* "important for me to devise a plan in order to have procedures for all stakeholders to participate"

* "collaborating with colleagues to set goals and achievable measures the school will be able to take ownership of the directive"

* "planning is essential for effective results"

Please reflect on the input provided above:

\section{You have determined that the current action plan put into} place at your school/department is falling short of expectations, you:

* $42.1 \%$ of participants chose: survey your leadership team to identify possible steps for improvement and utilize the input from stakeholders to develop a plan to reconstitute your efforts.

* $42.1 \%$ of participant chose: work with individuals in a trusted group of colleagues and brainstorm how to redirect your efforts.

\section{Participant Input:}

* "the leader needs to look at the part /parts the plan is failing to get a deeper understanding of the situation before consulting with the leadership team"

* "Consult similar school administrators that are meeting the expectations and implement some of their models that are effective

* "Stakeholders beyond the leadership team have to be included"

Please reflect on the input provided above: 
3. The pile of responsibilities on your desk never seems to decrease, you:

*57.9\% of participants chose: identify a strategic plan to take care of tasks with greater consequences and work with your leadership team to delegate duties.

\section{Participant Input:}

* " You don't have a 400 dollar a day employee answering the phone (when you can pay someone 90 a day to do that task"

* " Leadership is knowing how to delegate responsibilities in order to meet the demands and tasks"

* "Administration and the leadership team should work hand in hand with each other in order to make sure that tasks concerning the successful operation of the school are fulfilled"

* "Follow through has to be part of the plan as well"

\section{Please reflect on the input provided above:}

4. You are being asked to make a significant decision that will impact your school/department, you:

*42.1 of participants chose: highlight key points in possible choices and share these potential decisions with others to seek their input.

\section{Participant Input:}

* " You want your team to know you value their input"

* " Not everything requires input"

* " If you ask too many people for input you will have to many answers"

Please reflect on the input provided above: 
5. The enthusiasm that once existed due to taking on a new job has waned and it appears that you have not been able to perform up to the expectations that were set by yourself and others, you:

* 63.2\% of participants chose: reflect on the optimism that you have for your work ethic and devise a plan that will best serve the purpose that you

originally set out to achieve.

\section{Participant Input:}

* " Show support while also providing accountability"

* " Prioritizing you work then delegating is always an effective way to ensure work is not overlooked and deadlines are met"

* " It is important to delegate duties in order to complete all required tasks"

Please reflect on the input provided above:

6. It is December and an individual that you supervise has recorded 10 absences from work this school-year. This individual has just called and requested for three personal days. He/She has been open about family turmoil, you:

* $\mathbf{8 4 . 2 \%}$ of participants chose: work with the appropriate support services to help advise your colleague on possible options moving forward and to personally show support for him/her.

\section{Participant Input:}

* " The leader should work with the employee before involving others"

* "A unit is only as strong as its weakest link-- Consistent absences could lead to a critical interruption of services"

* Unfortunately sometimes in the system, we have to follow rules which may involve disciplinary consequences-kids have to come first

* " This is a difficult question but I am surprised at the results"

Please reflect on the input provided above: 
7. While closing the door to your office, you are reminded about your commitments to raising student achievement. State assessment scores from the previous school-year have just been published and they were lower than expected, you:

*57.9\% of participants chose: reflect on your experience, devise a plan to improve student performance, and put actions into place that will serve your overall mission.

\section{Participant Input:}

* " All improvement efforts should be strategic; and therefore, should be well researched, thought out, and thoughtfully implemented.

* " Reflection is key to continuous improvement"

* " I would take a deeper dive into data using Power BI and conduct individualized data chats with both quantitative and qualitative data points "

Please reflect on the input provided above:

8. You are preparing a statement to make to colleagues and over 300 community stakeholders, you:

* $\mathbf{5 7 . 9 \%}$ of participants chose: carefully write your announcement and share it with a trusted group of individuals to receive their input.

\section{Participant Input:}

* " Employees will feel valued if asked to provide input"

* " I tend to involve a smaller group of people in my decision making process"

* " I believe it is very important to have input from all stakeholders"

* " I believe reflection is needed -- even when things work you can make be more efficient with reflection"

Please reflect on the input provided above: 
9. Walking up and down the halls of your school/department, you have heard sharp criticisms of your approach to increase student achievement, you:

*63.2\% of participants chose: genuinely ask for the input of stakeholders to share possible areas for improvement and incorporate their responses into decisions going forward.

\section{Participant Input:}

* " By listening to the criticisms of stakeholders and being open to adjustments, administrators can become more effective in their role as leaders of those improvement efforts"

* " Continue with my approach until substantial evidence proves it effective or ineffective"

* " I understand that the role of an administrator is not always to be loved, but to be respected. There will never be a time when $100 \%$ of the stakeholders will be happy with your actions, but we must remember that the students come first"

\section{Please reflect on the input provided above:}

10. You just received a promotion and despite feeling honored by the increase in salary and responsibility, the challenges seem to be overwhelming, you:

*57.9\% of participants chose: know that challenges are expected and reinstate the belief that working hard and sharing a positive attitude will help you in overcoming these challenges.

\section{Participant Input:}

* " A positive mindset and an attitude of being willing to learn and overcome obstacles will assist in challenging moments until efficiency and efficacy take effect"

* " This is not an effective what to take on a promotion, knowing that the job may not be fulfilling, the individual should reconsider taking on the position-- the individual's priority is to receive a higher salary rather than have a job that is giving you a sense of satisfaction in all areas"

* "Just being positive and believing in myself, while it is helpful, is not enough."

* "Take up yoga!

Please reflect on the input provided above: 


\title{
VITA
}

\section{JOSEPH P. EBERHARD}

\author{
Born, Grand Forks, North Dakota
}

2003-2007

2007- 2014

2008-2010

B.A. Broadcast Journalism

American University

Washington, D.C.

Classroom Educator

Miami-Dade County Public Schools

Miami, Florida

M.S. Education Law

Nova Southeastern University

Davie, Florida

2011-2017

Doctoral Candidate

Educational Administration and Supervision

Florida International University

Miami, Florida

2014-2017

School-Site Administrator

Miami-Dade County Public Schools

Miami, Florida

2015-2017

Adjunct Professor

Educational leadership

Adrian Dominican School of Education, Barry University

Miami Shores, Florida

\section{PUBLICATIONS AND PRESENTATIONS}

Eberhard, J. (2013). School administration self-efficacy: Change-agents in an environment of turbulence. Paper presented at Florida Intentional University, The 12th Annual South Florida Education Research Conference, June 1, 2013, Miami, Florida. 